Pedro Caldana Gordon

\title{
Excitabilidade cortical motora como preditora de resposta na esquizofrenia
}

Tese apresentada à Faculdade de Medicina da Universidade de São Paulo para obtenção de título de Doutor em Ciências

Programa de Ciências Médicas

Área de concentração: Educação e Saúde

Orientador: Prof. Dr. Andre Russowski Brunoni

(Versão corrigida. Resolução CoPGr 6018/11, de 1 de novembro de 2011. A versão original está disponível na Biblioteca da FMUSP)

São Paulo

2018 
Dados Internacionais de Catalogação na Publicação (CIP)

Preparada pela Biblioteca da

Faculdade de Medicina da Universidade de São Paulo

Creprodução autorizada pelo autor

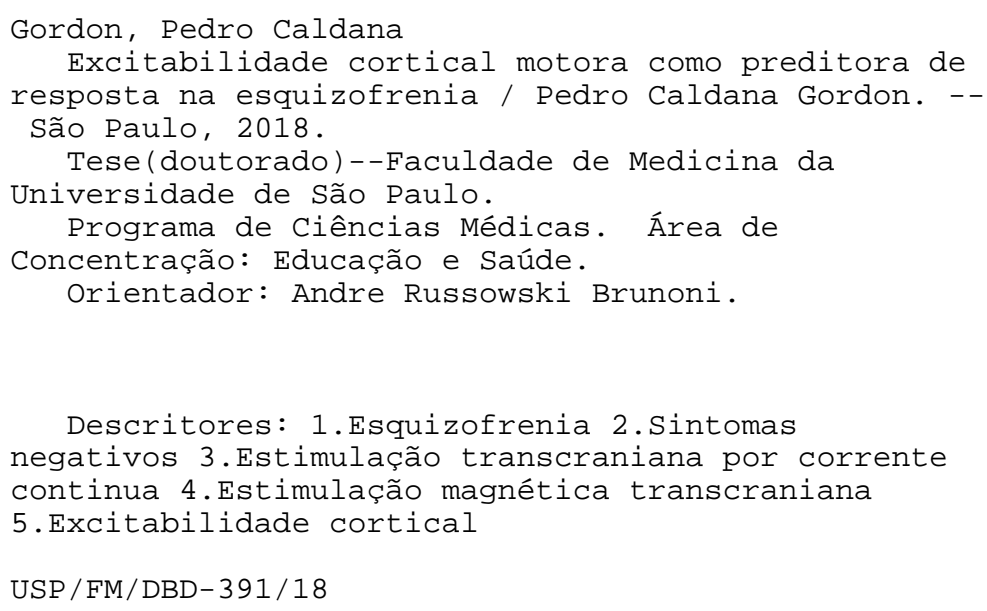

Responsável: Erinalva da Conceição Batista, CRB-8 6755 
Dedicatória

Aos meus pais, Beatriz e Carlos Alberto. 


\section{Agradecimentos}

Ao Prof. Dr. André Brunoni, meu orientador, pelo apoio e constante disponibilidade ao longo da realização desse trabalho.

A todos os membros do Serviço Interdisciplinar de Neuromodulação do Instituto de Psiquiatria, cuja dedicação e profissionalismo foram indispensáveis para a pesquisa.

A todos os voluntários da pesquisa e seus familiares, que se dispuseram a contribuir com o projeto, sem os quais esse trabalho não seria possível. 
Lista de siglas

Lista de tabelas

Lista de figuras

$\begin{array}{ll}\text { RESUMO } & 10\end{array}$

$\begin{array}{lr}\text { ABSTRACT } & 12\end{array}$

1. INTRODUÇÃO 14

1.1 Impacto da esquizofrenia $\quad \mathbf{1 4}$

1.2 Estimulação elétrica transcraniana por corrente contínua $\quad \mathbf{1 7}$ e sua utilização terapêutica

1.3 Medidas de excitabilidade cortical utilizando $\quad 21$ estimulação magnética transcraniana

2. MATERIAL E MÉTODOS 32

2.1 Objetivos $\quad 32$

2.2 Hipóteses $\quad 33$

2.3 Desenho do estudo $\quad 34$

2.4 Análise Estatística $\quad 44$

3. RESULTADOS 47

4. CONCLUSÕES

4.1 Limitações

5. SUGESTÕES PARA TRABALHOS FUTUROS $\mathbf{6 8}$

6. REFERÊNCIAS BIBLIOGRÁFICAS 70 


\section{LISTA DE SIGLAS}

\begin{tabular}{ll} 
DP & $\begin{array}{l}\text { Desvio padrão } \\
\text { Diagnostic and Statistical Manual of Mental Disorders } 5^{\text {th }} \text { edition } \\
\text { (Manual Diagnóstico e Estatístico de Transtornos Mentais } 5^{\text {a }} \text { edição) }\end{array}$ \\
ECM & Excitabilidade cortical motora \\
EEG & Eletroencefalografia \\
EMT & Estimulação magnética transcraniana \\
ETCC & Estimulação transcraniana por corrente contínua \\
GABA & gamma-Aminobutyric acid (acido gama-aminobutirico) \\
ICF & intra-cortical facilitation (Facilitação intracortical) \\
LM & Limiar Motor \\
LTP & long term potentiation (potencial de longo termo), \\
MADRS & $\begin{array}{l}\text { Montgomery-Åsberg Depression Rating Scale (Escala de depressão de } \\
\text { Montgomery-Åsberg) }\end{array}$ \\
ms & Milissegundo \\
PANSS & $\begin{array}{l}\text { Positive and negative syndrome scale (Escala de sintomas positivos e } \\
\text { negativos) }\end{array}$ \\
PEM & Potencial evocado motor \\
SANS & $\begin{array}{l}\text { Scale for the Assessment of Negative Symptoms (Escala para avaliação } \\
\text { de sintomas negativos) } \\
\text { short interval cortical inhibition (Inibição intracortical de intervalo } \\
\text { curto) }\end{array}$ \\
\hline
\end{tabular}




\section{LISTA DE TABELAS}

Página

TABELA $2.1-$ Sequência de procedimentos realizados durante a 37 participação no ensaio clínico STARTS

TABELA 3.1 - Dados Clínicos e Demográficos, e de excitabilidade cortical motora de 69 sujeitos do ensaio clínico ETCC para sintomas negativos de esquizofrenia, e comparação dos grupos ativo e placebo

TABELA 3.2 - Modelo de regressão linear multivariável incluindo todas as variáveis independentes no modelo, tendo como variável dependente a excitabilidade cortical motora (SICI em córtex esquerdo) em amostra de 69 sujeitos com esquizofrenia

TABELA 3.3 - Modelo de regressão linear multivariável incluindo todas

52 as variáveis independentes no modelo, tendo como variável dependente a excitabilidade cortical motora (SICI em córtex direito) em amostra de 69 sujeitos com esquizofrenia

TABELA 3.4 - Modelo de regressão linear multivariável incluindo todas as variáveis independentes no modelo, tendo como variável dependente a excitabilidade cortical motora (ICF em córtex esquerdo) em amostra de 69 sujeitos com esquizofrenia

TABELA 3.5 - Modelo de regressão linear multivariável incluindo todas as variáveis independentes no modelo, tendo como variável dependente a excitabilidade cortical motora (ICF em córtex direito) em amostra de 69 sujeitos com esquizofrenia 
TABELA 3.6 - Modelo de regressão linear multivariável com resultados

significativos, incluindo apenas variáveis clinicodemograficas significativamente correlacionadas com índices de excitabilidade cortical motora, em amostra de 69 sujeitos com esquizofrenia

TABELA 3.7 - Modelo linear de efeitos mixtos de medidas repetidas, com variaveis fixas tempo (pré e pós intervenção), intervenção (placebo e ETCC ativa) e hemisfério de medida da ECM (esquerdo e direito), tendo índices de ECM como variáveis independentes (modelo 1 com, SICI, modelo 2 com ICF) em amostra de 69 sujeitos com esquizofrenia 


\section{LISTA DE FIGURAS}

\section{Página}

FIGURA 1.1 - Modelo do funcionamento da EMT

FIGURA 1.2 - Variação da amplitude de resposta do PEM em

função da distância temporal entre o estímulo condicionante e o estímulo teste. (extraído de KUJIRAI et al. 1993)

FIGURA 2.1 - Simulação computacional do campo elétrico induzido por ETCC no córtex cerebral, utilizando de montagem descrita neste estudo. (extraído de Valiengo et al. no prelo)

FIGURA 3.1 - $\quad$ Fluxograma da triagem e seleção de sujeitos para o 46 ensaio clínico STARTS

FIGURA 3.2 - ECM por pulso pareado em amostra de 69 sujeitos com esquizofrenia antes e apos sessão de ETCC, divididos em intervenção ativa e intervenção placebo 


\section{RESUMO}

Gordon PC. Excitabilidade cortical motora como preditora de resposta na esquizofrenia [Tese]. São Paulo: Faculdade de Medicina da Universidade de São Paulo; 2018.

O desenvolvimento da estimulação magnética transcraniana (EMT) permitiu o estudo de potenciais evocados motores eliciados pela estimulação direta do córtex cerebral de forma nãoinvasiva. Foi observado que diferentes paradigmas de estimulação cortical por EMT apresentam diferentes padrões de resposta, que posteriormente foram associados ao funcionamento de circuitos corticais GABAérgicos e glutamatérgicos do córtex motor, compondo assim índices de excitabilidade cortical motora (ECM). Ademais, desvios da normalidade de tais índices foram encontrados em diversas condições clínicas, incluindo transtornos mentais como a esquizofrenia. O uso dessas medidas também auxiliou o desenvolvimento da estimulação transcraniana por corrente contínua (ETCC), técnica que se mostrou capaz de produzir efeitos neuromodulatórios no sistema nervoso central de forma segura e com mínimos efeitos adversos. Tal técnica vem apresentando possibilidades terapêuticas promissoras, como por exemplo, tendo sido observado sua eficácia no alívio de alucinações auditivas de indivíduos com esquizofrenia. O uso de ETCC para tratamento de sintomas negativos da esquizofrenia também pode vir a se mostrar uma abordagem eficaz, e a análise da ECM pode auxiliar no entendimento dos seus mecanismos de ação e atuar como possível preditor de resposta terapêutica.

O objetivo do presente estudo é avaliar o perfil de ECM em um grupo de indivíduos com esquizofrenia, e as possíveis influências de um protocolo terapêutico utilizando ETCC sobre essas medidas. Com esse objetivo, foi selecionada uma coorte de sujeitos com esquizofrenia que participou em ensaio clínico randomizado e controlado com placebo (estimulação sham), tendo a ETCC como intervenção ativa alvo. A ECM foi mensurada na avaliação inicial dos sujeitos, assim como após a primeira sessão de ETCC, e quando da avaliação de desfecho primário. O protocolo terapêutico de ETCC envolveu a colocação de 2 eletrodos de área $5 \times 7 \mathrm{~cm}$, pólo anódico aplicado sobre região correspondente ao córtex pré-frontal dorsolateral esquerdo e pólo catódico aplicado sobre córtex de transição temporoparietal esquerdo; com intensidade de corrente de $2 \mathrm{~mA}$, aplicada por 20 minutos. Cada sujeito foi submetido a 10 sessões no total.

Encontramos que idade se correlacionou com diminuição da inibição intracortical, reproduzindo resultado previamente encontrado em indivíduos saudáveis. Acerca da modulação da ECM após sessão de ETCC, observamos que sujeitos submetidos à intervenção ativa apresentaram aumento 
da inibição intracortical no hemisfério estimulado, em oposição à ausência de mudança significativa da ECM nos sujeitos que receberam estimulação placebo.

Os resultados sugerem que sessão de ETCC, utilizando os parâmetros aplicados neste estudo, levou ao aumento da inibição intracortical. Devido a evidências prévias de déficit de inibição intracortical em pessoas com esquizofrenia, é possível que o fenômeno observado represente mecanismo terapêutico da ETCC. É necessário verificar se tal efeito sobre a ECM acompanha medidas objetivas de resposta clinica. Caso isto se comprove, a ECM pode se tornar um valioso marcador de resposta terapêutica e evolução clinica em pacientes com esquizofrenia.

Descritores: Esquizofrenia; sintomas negativos; estimulação transcraniana por corrente continua; estimulação magnética transcraniana; excitabilidade cortical motora. 


\section{ABSTRACT}

Gordon PC. Motor cortical excitability as a response prediction in schizophrenia [Thesis]. São Paulo: "Faculdade de Medicina da Universidade de São Paulo"; 2018.

The development of transcranial magnetic stimulation allowed the study of motor evoked potentials by applying direct stimuli to the brain cortex in a non-invasive fashion. Different stimulation protocols were observed to yield different response patterns, which were later associated with the functioning of cortical GABAergic and glutamatergic circuits, assembled as motor cortex excitability indices. Also, deviations from normality of such indices were observed in several clinical conditions, including mental disorders such as schizophrenia. The use of these measurements also helped the development of transcranial direct current stimulation (tDCS), a technique which was shown to promote neuromodulatory effects in central nervous system, with potential treatment applications. This technique has been used with success in the treatment of auditory hallucinations in patients with schizophrenia. The use of tDCS might also be effective in the treatment of negative symptoms of schizophrenia, and motor cortex excitability analysis might be used to clarify its physiological effects and act as a possible treatment response predictor.

The aim of the present study is to evaluate the motor cortical excitability profile of individuals with schizophrenia, as well as possible influences of tDCS over these measurements. With this aim, we selected a cohort of subjects with schizophrenia who participated in a randomized placebo controlled clinical trial using transcranial direct current stimulation (and sham stimulation for placebo), and measuring motor cortical excitability during baseline evaluation, after the first stimulation session, and at the time of the primary outcome evaluation. The transcranial direct current stimulation protocol used in the present study involved the use of 2 electrodes of area $5 \times 7$ $\mathrm{cm}$, anode placed over the region corresponding to the left dorsolateral prefrontal cortex, and cathode over the left cortical temporoparietal juntion. A current of $2 \mathrm{~mA}$ intensity was applied for 20 minutes. Each subject underwent a total of 10 sessions.

We found that age was correlated to reduced intracortical inhibition, as has been previously found in healthy subjects. Regarding changes of motor cortical excitability following a transcranial direct current stimulation session, we observed that subjects that received the active stimulation displayed an increase in intracortical inhibition, as opposed to those who received sham stimulation, which did not present with any significant change.

Results suggest that transcranial direct current stimulation session, using the parameters described in this study, led to an increase in intracortical inhibition. Given previous evidence of intracortical 
inhibition deficit in individuals with schizophrenia, it is possible that the observed phenomenon corresponds to a treatment mechanism of the electrical stimulation in this population. This need to be confirmed by comparing such changes in cortical excitability to objective measurements of clinical improvement. In case that is confirmed, measurement of motor cortical excitability may have a valuable application as a marker of treatment response and clinical outcome for patients with schizophrenia.

Descriptors: Schizophrenia; negative symptoms; transcranial direct current stimulation; transcranial magnetic stimulation; motor cortical excitability. 


\section{INTRODUÇÃO}

\subsection{Impacto da Esquizofrenia}

Esquizofrenia é um transtorno mental de impacto significativo para o indivíduo acometido e para a sociedade. A prevalência da doença ao longo da vida na população geral é estimada em torno de 0.5 a $1 \%$, valores que se mantém estáveis em diferentes populações estudadas (Saha, Chant, Welham, \& McGrath, 2005). Ademais, é considerada uma doença altamente incapacitante, sendo responsável por um grande impacto econômico sobre a população, seja pela perda de anos de trabalho por incapacidade, ou devido ao uso de recursos em saúde (Chong et al., 2016).

Clinicamente os sintomas da esquizofrenia são divididos em três categorias: sintomas positivos, sintomas negativos e sintomas cognitivos. Sintomas positivos se referem a fenômenos que habitualmente não são observados em pessoas saudáveis; envolvendo experiências sensoriais na ausência de um percepto real (alucinações), alterações patológicas do pensamento, que geralmente acompanham a formação de crenças irreais e não compartilhadas (delírios) e, frequentemente, alteração do comportamento, com o paciente assumindo atitude paranóide, agressiva ou francamente bizarra (Sadock, Sadock, Ruiz, \& Kaplan, 2009). A abordagem recomendada para esse grupo de sintomas, e todos os indivíduos com o diagnóstico de esquizofrenia, envolve a administração de medicações antipsicóticas, considerado o tratamento padrão para a doença (Freedman, 2003). Apesar da eficácia comprovada do tratamento antipsicótico para pacientes com esquizofrenia, uma proporção significativa destes não apresenta resposta clínica adequada, sendo em torno de $30 \%$ dos pacientes considerados refratários 
em algum estágio do tratamento (Brenner et al., 1990). Para essa população o tratamento de escolha envolve a medicação Clozapina, reservada apenas para esses casos devido a seus efeitos adversos potencialmente graves (Buckley et al., 2001).

Não obstante tais estratégias terapêuticas, ou mesmo em casos de alívio dos sintomas positivos, muitos indivíduos acometidos mantém grave comprometimento da qualidade de vida, apresentando prejuízo no desempenho profissional, acadêmico, assim como dificuldade em relacionamentos sociais diversos (Ucok \& Ergul, 2014). De fato, uma proporção significativa dos pacientes apresenta progressão da doença com o passar do tempo, mesmo naqueles que apresentam resposta adequada ao tratamento para os sintomas positivos, sendo observada perda acelerada de volume cerebral com o avançar da idade em pacientes com esquizofrenia comparados a indivíduos saudáveis (van Haren, Cahn, Hulshoff Pol, \& Kahn, 2008). Estima-se que outro conjunto de sintomas, chamados sintomas negativos, sejam os maiores responsáveis por tal prejuízo da qualidade de vida e da funcionalidade observados em pacientes com esquizofrenia (Fervaha, Foussias, Agid, \& Remington, 2014; Rabinowitz et al., 2012).

Se entendermos os sintomas positivos como o desenvolvimento patológico de percepções e comportamentos considerados anormais, podemos pensar nos sintomas negativos então como uma ausência patológica de sensações e comportamentos considerados normais. Mais precisamente, sintomas negativos são descritos como a redução ou ausência de comportamentos considerados normais, incluindo a diminuição global da emissão de comportamentos dirigidos a objetivos (Sarkar, Hillner, \& Velligan, 2015). Tal fenômeno se apresenta nos indivíduos acometidos como retraimento social, diminuição da resposta afetiva a estímulos, diminuição de interesse pelo seu entorno e por 
atividades em geral (sociais, laborais, e em casos mais avançados, atividades quaisquer que sejam) (Sarkar et al., 2015). Com a evolução da doença, o impacto dos sintomas negativos sobre o indivíduo leva a grave isolamento social, perda de capacidade de comunicação e dos cuidados pessoais; compreensivelmente impondo um prejuízo significativo em sua vida diária. Ademais, pela natureza dos sintomas negativos, sujeitos acometidos tendem a perder o interesse pela própria saúde, prejudicando a aderência ao tratamento e impondo mais um desafio para o manejo clínico (Sarkar et al., 2015).

Diferentemente dos sintomas positivos, as opções de abordagens terapêuticas para os sintomas negativos são escassas. O uso de diversas formas de tratamentos farmacológicos, usualmente com antidepressivos, novas classes de medicações antipsicóticas ou outras classes de medicações; assim como modalidades de tratamento psicoterápico; tem apresentado resultados insatisfatórios no tratamento desses sintomas (Sarkar et al., 2015). Até o momento, nenhuma modalidade terapêutica é considerada consistentemente eficaz no tratamento dos sintomas negativos da esquizofrenia (Tsapakis, Dimopoulou, \& Tarazi, 2015).

Nesse contexto, a compreensão de mecanismos fisiopatológicos envolvidos na doença e nos sintomas negativos poderia guiar o desenvolvimento de alternativas terapêuticas eficazes para o transtorno. Por exemplo, meta-análise envolvendo estudos de ressonância magnética funcional revelou consistente diminuição da atividade cortical em determinadas regiões cerebrais, acometendo de forma mais importante o córtex pré-frontal dorsolateral e porção anterior do giro do cíngulo (Minzenberg, Laird, Thelen, Carter, \& Glahn, 2009). 
Baseando-se nesses resultados de neuroimagem, métodos de neuromodulação nãoinvasiva foram utilizados na tentativa de estimular a atividade cortical dessas áreas, objetivando possível alívio de sintomas, mais precisamente pela realização de sessões de estimulação magnética transcraniana (EMT) repetitiva em regiões pré-frontais. Entretanto, os resultados de ensaio clínico utilizando esse método foram pouco animadores (Barr, Farzan, Tran, Fitzgerald, \& Daskalakis, 2012).

Recentemente foram publicados ensaios clínicos utilizando estimulação transcraniana por corrente contínua (ETCC) para o tratamento de sintomas da esquizofrenia, focando especialmente no controle de alucinações auditivas, tendo estes sugerido sucesso no alívio do sintoma (Brunelin et al., 2012; Frohlich et al., 2016). Um destes estudos, de maior amostra, também revelou que sujeitos que receberam tratamento ativo apresentaram redução significativa dos sintomas negativos, comparados ao grupo placebo (Brunelin et al., 2012). Dessa forma, faz-se necessária a reprodução desse resultado, preferencialmente por meio de ensaio clínico objetivando especificamente a pesquisa dos resultados da ETCC sobre esse conjunto de sintomas. Caso prove ser um tratamento eficaz no alívio de sintomas negativos, a ETCC pode se tornar uma ferramenta de grande valor no arsenal terapêutico para pessoas com esquizofrenia.

\subsection{Estimulação elétrica transcraniana por corrente contínua e sua}

\section{utilização terapêutica}

Embora o uso de corrente elétrica para obter-se efeito neurobiológico e terapêutico date de séculos, apenas recentemente estudos têm investigado detalhadamente os mecanismos de ação dessas técnicas. Um dos primeiros trabalhos dessa nova era foi o de 
Nitsche e Paulus (Nitsche \& Paulus, 2000) que descreveu efeitos neuromodulatórios da ETCC em humanos. Assim como muitos trabalhos subsequentes utilizando esta e outras técnicas de neuromodulação não-invasivas, este estudo utilizou a análise da excitabilidade cortical motora (ECM) para compreender os efeitos da intervenção sobre o sistema nervoso. Assim foi demonstrado que a aplicação de uma corrente elétrica de intensidade 1 mA, por meio de um eletrodo colocado na superfície do escalpe na região correspondente ao córtex motor primário, leva a alterações da ECM. Mais precisamente, observou-se que após estimulação com eletrodo anódico houve aumento da facilitação intracorical, e após estimulação com eletrodo catódico houve aumento da inibição intracortical (Nitsche et al., 2003; Nitsche \& Paulus, 2000). A forma como são medidos esses parâmetros será explicada na sessão seguinte.

Para compreendermos como a ETCC altera a atividade cortical é necessário inicialmente considerar que a corrente elétrica induzida é insuficiente para provocar diretamente potenciais de ação no córtex cerebral, ou qualquer região do sistema nervoso central (Stagg \& Nitsche, 2011). Mesmo assim, o campo elétrico induzido tem ação sobre a atividade do tecido nervoso. A polaridade negativa leva à diminuição da diferença de potencial dos neurônios, que consequentemente ficam em estado de maior probabilidade de despolarização; e o oposto ocorre com polaridades positivas (Stagg \& Nitsche, 2011). Além desse efeito observado durante a aplicação do campo elétrico, a exposição do tecido neuronal a este traz alterações de duração prolongada mesmo após o término da estimulação, sendo observado maior arborização dendrítica e expressão de fatores neurotróficos no tecido cerebral; reforçando o efeito neuromodulatório da ETCC (Pelletier \& Cicchetti, 2014). 
Tal experimento foi repetido, porém associando a estimulação ao uso de fármacos com ação sobre o sistema nervoso, tendo sido observado que substâncias que aumentam a taxa de potenciais de ação (agonistas NMDA) potencializam a facilitação provocada pela ETCC, e substâncias que diminuem a taxa de potenciais de ação (carbamazepina) tem efeito contrário (Liebetanz, Nitsche, Tergau, \& Paulus, 2002). Estudos subsequentes confirmaram tais observações, reforçando o modelo em que a ETCC provoca efeito neuroplástico ao modular a probabilidade de despolarização de tecido neuronal, com polaridades negativas levando ao fenômeno de long term potentiation (LTP - potencial de longo termo), implicado no aumento das conexões sinápticas de um circuito e consequente facilitação da despolarização neuronal, e polaridades positivas inibindo esse fenômeno (Stagg \& Nitsche, 2011). Seguindo essa linha, estudo de modelo animal induziu esse mesmo fenômeno em tecido hipocampal, que após ser submetido a ação de campo elétrico em contato com polo anódico, demonstraram resposta eletrofisiológica e expressão gênica compatíveis com LTP, mesmo em uma intensidade de campo elétrico insuficiente para provocar diretamente potenciais de ação (Ranieri et al., 2012).

A importância da comprovação do efeito neuromodulador de estimulação elétrica de baixa intensidade, mesmo em níveis incapazes de gerar potenciais de ação em córtex cerebral, reside no fato de ser possível realizar tal procedimento em sujeitos humanos com objetivo de intervir no funcionamento cortical, possivelmente com fins terapêuticos, de forma altamente segura e tolerável (Antal et al., 2017).

\section{Método de aplicação da ETCC}

O equipamento da ETCC é constituído por uma bateria, amperímetro, potenciômetro e eletrodos (um ânodo e um cátodo). Os eletrodos são colocados sobre a superfície do 
corpo, fechando o circuito e permitindo a passagem de corrente. Os componentes do equipamento mensuram a impedância do circuito, incluindo os tecidos biológicos nos quais a corrente percorre (couro cabeludo, crânio, encéfalo), e consequentemente permite fixar uma determinada intensidade da corrente elétrica que se deseja utilizar, possível pela variação da diferença de potencial (Lei de Ohm).

A ETCC é considerada um procedimento seguro e virtualmente isento de efeitos adversos graves, sendo os efeitos mais reportados sensação de formigamento no local de aplicação e sensação de fadiga após a sessão, ambos de curta duração (Antal et al., 2017; Poreisz, Boros, Antal, \& Paulus, 2007). Lesões da pele na região de aplicação foram reportadas em algumas ocasiões, embora tais casos tenham sido relacionados à aplicação inadequada de solução condutora entre o eletrodo e a pele (Palm et al., 2008). Lesão de tecido nervoso foi induzida em modelos animais apenas quando utilizadas densidades de corrente acima de $140 \mathrm{~A} / \mathrm{m}^{2}$, ou seja, mais de 200 vezes superior àquelas utilizadas habitualmente (Liebetanz et al., 2009).

Os mínimos efeitos adversos associados à técnica, somados à não-invasividade e baixo custo, sugerem que tal técnica possa se tornar uma relevante ferramenta terapêutica (Brunoni et al., 2012). Recentemente, ensaios clínicos randomizados tem aportado evidências do benefício terapêutico dessa técnica para algumas afecções como depressão maior (Brunoni et al., 2017; Brunoni et al., 2013), sintomas não-motores da doença de Parkinson (Manenti et al., 2016) e alucinações auditivas em esquizofrenia (Brunelin et al., 2012).

Métodos de ECM, além de terem auxiliado na compreensão do mecanismo de ação da ETCC, podem também vir a ser utilizados em ensaios clínicos envolvendo esse 
tratamento. Tal método pode trazer informações relevantes sobre os efeitos terapêuticos em diferentes afecções, avançando os conhecimentos sobre estas e auxiliando no desenvolvimento de tratamentos mais eficazes.

\subsection{Medidas de Excitabilidade Cortical utilizando Estimulação}

\section{Magnética Transcraniana}

Em 1985 Barker e colaboradores demonstraram ser possível a estimulação do córtex cerebral por meio de indução eletromagnética (Barker, Jalinous, \& Freeston, 1985), técnica chamada de Estimulação Magnética Transcraniana (EMT). Tal estimulação é possível devido aos mecanismos envolvidos no eletromagnetismo, cujas leis foram unificadas pelo cientista James Clerk Maxwell, e descrevem que a passagem de uma corrente elétrica por um meio (no caso da EMT seria o circuito da bobina) induz a formação de um campo magnético de vetor ortogonal à direção da corrente elétrica; e que a variação no tempo da intensidade de um campo magnético induz a formação de um campo elétrico de vetor ortogonal ao do campo magnético. Dessa forma, o rápido surgimento de uma corrente elétrica de alta intensidade pela bobina posicionada sobre a superfície craniana é capaz de gerar um campo elétrico no córtex cerebral, com intensidade suficiente para despolarizar os neurônios dessa região. A depender da função atribuída ao córtex cerebral no qual essa estimulação é aplicada, os efeitos observados serão diferentes. Por exemplo, aplicação sobre o córtex occipital pode provocar a percepção de escotomas no campo visual do sujeito, e sobre o córtex motor primário pode eliciar respostas motoras no dimídio contralateral. A figura 1.1 expõe um modelo simplificado dos princípios físicos básicos da EMT. 
FIGURA 1.1 - Modelo do funcionamento da EMT
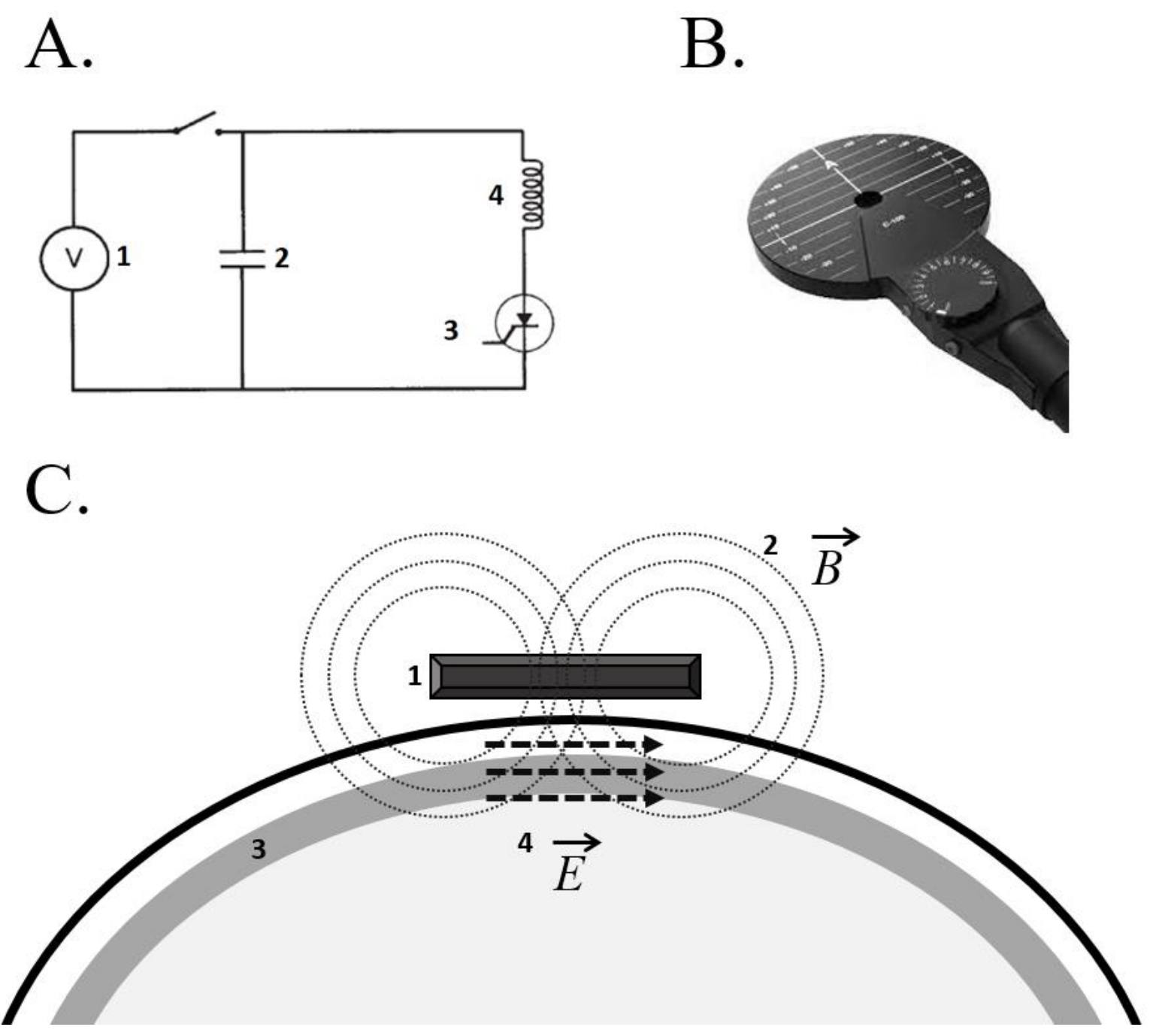

A. Circuito que compõe o aparelho de EMT. Quando o aparelho é ligado a uma fonte elétrica capaz de gerar diferenças de potencial (1), o capacitor (2) é carregado. Em seguida, quando o tiristor (3) é acionado, a carga armazenada no capacitor gera uma corrente que percorre a bobina (4), induzindo um campo magnético ao seu redor.

B. Fotografia de uma bobina circular EMT utilizada no Serviço Interdisciplinar de Neuromodulação do IPq-HCFMUSP, utilizada neste estudo.

C. Esquema do mecanismo de EMT sobre o córtex cerebral. O surgimento de uma corrente elétrica no circuito da bobina de EMT (1) induz à formação de um campo magnético $\vec{B}$ (2), cuja variação no tempo induz a formação de um campo elétrico $\vec{E}$. O campo magnético atravessa a superfície do crânio sem alteração, atingindo o córtex cerebral (3), e então o campo elétrico induzido (4) leva à despolarização dos neurônios da região. 
O exato mecanismo biofísico pelo qual o campo elétrico induzido pela EMT despolariza os neurônios do córtex cerebral não é completamente conhecido. A principal hipótese é que o estímulo leva à despolarização da região axonal dos neurônios pois, devido à alta densidade de canais iônicos nessa região, esta seria mais susceptível à despolarização provocada pela diferença de potencial causada pelo campo elétrico induzido e, consequentemente, gerando potenciais de ação (Dayan, Censor, Buch, Sandrini, \& Cohen, 2013). Esse modelo corresponde com as observações de Di Lazzaro et al. (Di Lazzaro et al., 2004), que descreveram que a estimulação utilizando uma bobina "em formato de 8", cuja configuração permite a indução de um campo elétrico uniforme de maior amplitude no centro da aplicação, é capaz de eliciar respostas motoras de intensidades e características diferentes, dependendo da direção do vetor do campo elétrico. A conclusão é que alterando a angulação do vetor do campo elétrico, este transpassa diferentes fibras de axônios intracorticais, induzindo assim respostas motoras de características diferentes, sugerindo que se trata da região do tecido nervoso sobre o qual os pulsos de EMT tem maior impacto (Di Lazzaro et al., 2004).

Assim, é possível estudar in vivo respostas do tecido neuronal do córtex cerebral a diferentes estímulos, de forma não invasiva. A aplicação de estímulos sobre o córtex motor e a mensuração do potencial evocado motor (PEM) por meio de um eletromiógrafo de superfície permite quantificação indireta do efeito da estimulação cortical. Dessa forma foi observado que variando a intensidade do estímulo podem-se eliciar respostas motoras de amplitudes crescentes (van der Kamp, Zwinderman, Ferrari, \& van Dijk, 1996). É possível realizar a calibragem da intensidade do estímulo da EMT, selecionando no aparelho a porcentagem da carga total do capacitor que se deseja descarregar (ver figura 
1.1 A), alterando assim a intensidade da corrente e consequentemente do campo magnético e, então, do campo elétrico induzidos (ver figura 1.1 C).

Utilizando o aparelho dessa forma, é possível encontrar a intensidade mínima capaz de eliciar uma resposta motora, intensidade chamada de limiar motor (LM). É consenso que o LM é a intensidade mínima do aparelho, mensurado em porcentagem da carga total do capacitor, capaz de eliciar uma resposta motora de amplitude de ao menos $50 \mu \mathrm{V}$ em ao menos $50 \%$ dos estímulos aplicados, no caso de aferição sobre o músculo abductor pollicis brevis (Chen et al., 2008; Rossini et al., 1999). A medida desse parâmetro é importante para diversos parâmetros de medidas da excitabilidade cortical utilizando EMT, descritas a seguir.

O uso do PEM por meio de um estímulo teste único foi cogitado como uma possível forma de verificar o quão excitável estaria o córtex frente a um estímulo, quantificando o fenômeno por meio da amplitude da resposta motora. Entretanto a amplitude do PEM por estimulo único se mostrou altamente variável não apenas entre sujeitos, mas também no mesmo indivíduo em longo prazo. Diversas variáveis foram implicadas na variação do PEM por pulso único e, mesmo assim, não explicam a maior parte da variabilidade do potencial evocado, tornando-se difícil o uso do PEM por pulso único como instrumento isolado em estudos neurofisiológicos (Burke, Hicks, Stephen, Woodforth, \& Crawford, 1995; Rosler et al., 2002). Apesar da limitação do uso dessa medida para comparação entre diferentes sujeitos, estudos utilizaram com sucesso a comparação do PEM intrasujeito antes e após uma intervenção por meio da razão "PEM pós" sobre "PEM pré" determinada intervenção. Por exemplo, observou-se que sujeitos realizando treinamento muscular apresentaram menor razão do PEM quando faziam uso de benzodiazepínicos 
comparado aos que não utilizaram a substância (Ziemann, Muellbacher, Hallett, \& Cohen, 2001). De forma análoga, o uso de psicoestimulantes aumentou a razão do PEM (Ziemann, Tam, Butefisch, \& Cohen, 2002). A conclusão desses estudos é que a diferença de amplitude do PEM reflete as propriedades neuroplásticas de uma determinada intervenção: treinamento motor provoca facilitação intracortical do córtex motor por indução de LTP, traduzido por aumento da amplitude do PEM por pulsos de EMT; e que tal efeito neuroplástico é então potencializado com o uso de psicoestimulantes (com maiores razões de PEM traduzindo efeito neuroplástico do treinamento sendo realçado pela medicação), e é inibido por agonistas GABAérgicos (com menores razões de PEM traduzindo redução do efeito neuroplástico do treinamento pela medicação) (Ziemann et al., 2008). Ademais, outras medidas de ECM utilizando EMT foram desenvolvidas, capazes de traduzir mais nuances do funcionamento cortical.

\section{Pulso Pareado}

Efeitos da aplicação de dois pulsos sequenciais de EMT sobre o córtex motor, e seu impacto na amplitude do PEM, foram primeiramente descrito por Valls-Solé e colaboradores (Valls-Sole, Pascual-Leone, Wassermann, \& Hallett, 1992). Observou-se que um estímulo teste, de intensidade acima do LM, quando brevemente antecedido por um estimulo condicionante de intensidade abaixo do LM, tem sua amplitude de resposta alterada. Ademais, verificou-se que a amplitude da resposta varia em função da distância temporal entre o estímulo teste e o estímulo condicionante.

Kujirai e colaboradores (Kujirai et al., 1993) avançaram nas observações, detalhando a relação entre o intervalo entre estímulos do pulso pareado e a mudança na amplitude do PEM. Observou-se que quando o estímulo condicionante antecede o estímulo teste em 1 a 
5 ms, ocorre diminuição da amplitude do potencial evocado motor, fenômeno alcunhado de "inibição intracortical de intervalo curto" (SICI), e quando o estímulo condicionante antecede o estímulo teste em 10 a 15 ms observa-se um aumento da amplitude, fenômeno alcunhado de "facilitação intracortical" (ICF). A figura 1.2 exibe um gráfico que ilustra esses fenômenos.

FIGURA 1.2 - Variação da amplitude de resposta do PEM em função da distância temporal entre o estímulo condicionante e o estímulo teste. (extraído de KUJIRAI et al. 1993)

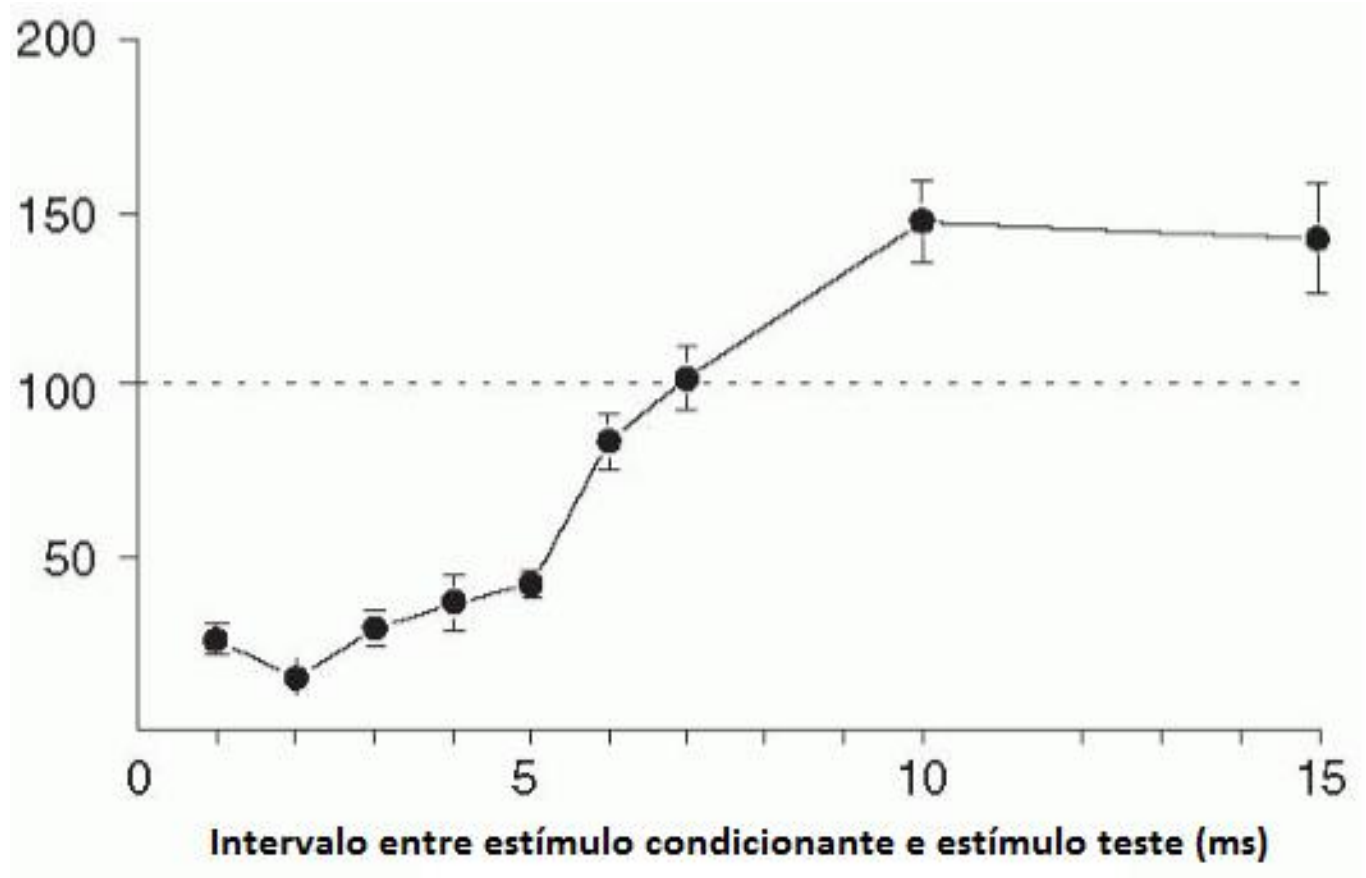

A abcissa do gráfico corresponde ao intervalo de tempo entre ambos os estímulos (ISI). A ordenada corresponde à razão (em porcentagem) entre a amplitude do PEM de um estimulo teste, quando antecedido por um estímulo condicionante, sobre a amplitude do PEM de um estimulo único.

Esse conjunto de fenômenos, primeiramente observados em músculos interrósseos dos membros superiores, também foram reproduzidos em diversas outras projeções 
musculares, tanto em membros inferiores quanto membros superiores, reforçando a hipótese de que a origem do fenômeno ocorre em nível intracortical e independe da força das projeções corticoespinhais (Chen et al., 1998). A potência do estímulo condicionante, no entanto, interfere diretamente no fenômeno, sendo observado máximo SICI quando o estímulo condicionado tem potencia entre 70 e $80 \%$ do LM (ou seja, maior diminuição da amplitude do potencial evocado por estímulo teste que sucedeu um estímulo condicionante, comparado com o potencial evocado por um estímulo único) (Kossev, Siggelkow, Dengler, \& Rollnik, 2003; Kujirai et al., 1993).

Di Lazzaro e colaboradores (Di Lazzaro et al., 1999) realizaram mensuração invasiva para estudar o PEM por pulsos pareados de EMT, utilizando eletrodos epidurais implantados na medula cervical de pacientes em tratamento de dor crônica refratária. Observou-se que o estímulo condicionante não provocou nenhuma resposta, o que é esperado dado que a intensidade do estímulo é abaixo do LM. Entretanto, o estímulo teste provocou o surgimento de ondas-D captadas pelos eletrodos epidurais, que correspondem à descarga de axônios do trato corticoespinhal em resposta à ativação direta dos neurônios piramidais do córtex motor primário, e que foi imediatamente sucedida pelo surgimento de ondas-I, que correspondem ao potencial de ação de neurônios piramidais quando ativados por projeções neuronais excitatórias (Di Lazzaro et al., 1999). Em seguida, observou-se que a pesquisa do SICI com um estímulo condicionante não alterou a amplitude da ondaD, porém diminuiu significativamente a amplitude das ondas-I subsequentes, fortalecendo a hipótese de que o fenômeno de inibição da amplitude do PEM pelo pulso pareado é produto de interações que se dão no nível do córtex cerebral. A mesma constatação foi 
feita para a o fenômeno de facilitação do PEM por pulso pareado, a ICF (Hanajima et al., 2002).

Estudos dos efeitos de fármacos sobre paradigmas de pulso pareado foram importantes para elucidar mecanismos fisiológicos da facilitação e inibição intracortical. Observou-se que a administração de benzodiazepínicos leva ao aumento do fenômeno de SICI (correspondendo a diminuição da amplitude do pulso pareado em comparação com pulso teste) (Di Lazzaro, Oliviero, et al., 2005; Di Lazzaro, Pilato, Dileone, Tonali, \& Ziemann, 2005). Esses estudos observaram maior aumento do SICI com administração de lorazepam, de maior afinidade por receptores $\mathrm{GABA}_{\mathrm{A}}$, comparado ao diazepam, de maior afinidade por receptores $\mathrm{GABA}_{\mathrm{B}}$. Concomitantemente, um estudo não observou aumento SICI após administração de baclofeno, medicação GABA $A_{B}$ agonista (McDonnell, Orekhov, \& Ziemann, 2006). Tais achados sugerem que o SICI seja causado pela atividade de interneurônios inibitórios GABAérgicos tipo A de resposta rápida, com maior atividade dessa rede interneuronal inibitória correspondendo com menor amplitude da PEM eliciado por tal paradigma de pulso pareado. Dessa forma, sugeriu-se que a utilização de paradigmas que pesquisem o SICI seria uma maneira de perscrutar a atividade dessa rede de interneurônios corticais.

Seguindo essa linha, foram utilizados paradigmas de pulso pareado em pacientes acometidos por diversas doenças e comparados os resultados aos de sujeitos saudáveis, com achados relevantes. Pacientes com distonias generalizadas (Gilio et al., 2000) e formas focais de distonia, como blefaroespasmo, câimbra do escritor (Sommer et al., 2002), e torcicolo espasmódico (Hanajima et al., 1998) apresentaram redução significativa do SICI. Tais resultados estão de acordo com os modelos teóricos sobre distonias, 
descritas como condições associadas à falha da inibição do aparelho de controle motor do sistema nervoso central. O achado de diminuição do SICI sugere que de fato há alteração da excitabilidade cortical, com hipofunção de neurônios inibitórios e consequente hiperativação das vias motoras, levando a atividade motora exacerbada e anormal. Curiosamente, estudo de Hanajima et al. (Hanajima et al., 1999) sugere que não há alteração do SICI em pacientes portadores de coréia, transtorno do movimento secundário a lesões em gânglios da base, ou seja, que não afetam diretamente o córtex cerebral.

Da mesma forma que o SICI seria causado pela atividade de interneurônios inibitórios, supôs-se que a facilitação intracortical mensurada por pulsos pareados de ISI acima de $8 \mathrm{~ms}$, seria causado por interneurônios excitatórios. Estudos farmacológicos corroboram parcialmente com esta hipótese, com sujeitos que receberam tratamento com memantina (antagonistas de receptores NMDA) apresentando diminuição do ICF comparados com placebo (Schwenkreis et al., 1999). Análogo aos estudos farmacológicos, um estudo com sujeitos portadores de variações gênicas de receptor de NMDA apresentaram alterações significativas do ICF comparados com controles (Mori et al., 2011). Entretanto, a relação do ICF com mecanismos intracorticais não é tão clara. Por exemplo, estudos invasivos observaram que variações do ICF não são resultado direto da variação da amplitude das ondas-I das projeções corticoespinais como no caso do SICI, sugerindo assim que esse fenômeno seja causado por mecanismos mais complexos que apenas uma relação linear com a atividade de interneurônios excitatórios (Di Lazzaro et al., 2006).

Excitabilidade cortical por pulso pareado também foi utilizada para o estudo de transtornos mentais, comparando as medidas de sujeitos afetados com as de controles 
normais. Em meta-análise dessa técnica utilizada em diversos transtornos mentais, encontrou-se que os transtornos que apresentam alterações mais consistentes de ECM foram depressão maior, transtorno obsessivo-compulsivo e esquizofrenia, todos associados à diminuição do SICI, sugerindo disfunção cortical por hipofunção da atividade GABAérgica (Radhu et al., 2013). A presença de alteração de marcadores com mecanismos fisiológicos identificados em pacientes com transtornos mentais abre a possibilidade de ser utilizado como possível biomarcador de doença e de resposta terapêutica.

Uma limitação relevante mencionada por estudos da área se refere à dependência de mensurar um potencial evocado motor como marcador de transtornos mentais que, a priori, não envolvem fenômenos motores. Um dos argumentos a favor do uso desses métodos é a existência de alterações de sensibilidade a receptores que afetariam o córtex como um todo, promovendo quadro clínico cognitivo-comportamental em conjunto com efeitos mensuráveis em córtex motor, permitido o uso dessas técnicas como medida indireta do funcionamento cortical (Daskalakis, Christensen, Fitzgerald, \& Chen, 2002). De fato, um estudo demonstrou que indivíduos com esquizofrenia sem queixas motoras apresentam déficit de neuromodulação em córtex motor, sugerindo que disfunções da doença não estariam limitadas a apenas algumas regiões do córtex e são passiveis de estudo com técnicas que usam PEM (Frantseva et al., 2008).

Uma alternativa que tem ganhado espaço recentemente é o estudo de potencial evocado por EMT avaliado no nível cortical por sinais de EEG (EMT-EEG), permitindo a observação de diferentes respostas corticais a estímulos em qualquer região do córtex, sem a dependência da mensuração da resposta motora (Daskalakis, Farzan, Radhu, \& 
Fitzgerald, 2012; Hill, Rogasch, Fitzgerald, \& Hoy, 2016; Ilmoniemi \& Kicic, 2010). Corroborando com a validade dessa técnica, foi observada uma grande associação entre respostas de protocolos de excitabilidade cortical por EMT analisando o PEM e a resposta por sinais de EEG (Daskalakis et al., 2008; Farzan et al., 2013). Assim, apesar de ser um método indireto de mensuração da excitabilidade cortical em sujeitos com condições neuropsiquiátricas, e do desenvolvimento recente de métodos alternativos, a pesquisa de ECM por EMT demonstrou associação com atividade cortical de regiões não motoras, além de possuir um corpo de evidência sugerindo seus mecanismos de ação, assim como alterações dessas medidas em condições neuropsiquiátricas (Radhu et al., 2013). 


\section{MATERIAL E MÉTODOS}

\subsection{Objetivos}

O estudo tem como objetivo geral a investigação de medidas de ECM utilizando EMT como possíveis marcadores da resposta à terapia biológica ETCC em pacientes portadores de esquizofrenia. Para esse fim, foram selecionados sujeitos que participaram de ensaio clínico envolvendo ETCC como intervenção para tratamento de sintomas negativos da esquizofrenia.

Como objetivos específicos, estão incluídos: 1) Identificação dos padrões de ECM associados a diferentes grupos de sintomas e gravidade em sujeitos com esquizofrenia. 2) Identificação dos padrões de ECM passíveis de alteração após a aplicação de ETCC em sujeitos com esquizofrenia. 3) Identificação dos padrões de ECM associados a resposta terapêutica e evolução clínica após ETCC em sujeitos com esquizofrenia. 


\subsection{Hipóteses}

1. Medidas de ECM apresentarão correlação com gravidade de sintomas dos pacientes com esquizofrenia, mensurada pela escala de sintomas positivos e negativos (PANSS). Especificamente, gravidade mensurada pela PANSS estará inversamente correlacionada com níveis de inibição intracortical medidos pelo SICI.

2. Sujeitos com esquizofrenia submetidos a sessão de ETCC apresentarão maiores mudanças em medidas de ECM comparados aos sujeitos que realizarem intervenção placebo. Especificamente, será observado aumento dos níveis de inibição intracortical medido pelo SICI, correspondendo a normalização do funcionamento cortical nessa população.

3. Evolução da gravidade de sintomas negativos após o tratamento, mensurada pela PANSS, apresentará correlação com a alteração dos níveis de ECM. Especificamente, melhora clínica estará correlacionada com aumento dos níveis de inibição intracortical medido pelo SICI. 


\subsection{Desenho do estudo}

O presente trabalho foi contido dentro de ensaio clínico, randomizado, duplo-cego e controlado com placebo, tendo este como objetivo o estudo de uma coorte de sujeitos com diagnóstico de esquizofrenia, submetidos a uma intervenção terapêutica experimental, ETCC (Valiengo et al. no prelo). Como parte das avaliações do ensaio clínico, os participantes foram submetidos a medidas de ECM utilizando EMT quando da avaliação inicial, após primeira sessão de ETCC, assim como após o tratamento.

De acordo com procedimento do ensaio clínico, os sujeitos foram divididos em dois grupos, um grupo que realizou a intervenção ativa e um grupo que realizou uma intervenção placebo (estimulação sham), permitindo a observação dos efeitos da intervenção ativa sobre a ECM comparados com intervenção placebo.

\section{Ensaio Clínico - STARTS}

O objetivo principal do ensaio clínico em questão foi verificar a efetividade da modalidade terapêutica ETCC para o tratamento de sintomas negativos em indivíduos portadores de esquizofrenia (Valiengo et al. no prelo).

O ensaio clínico foi aprovado pelo Comitê de Ética em Pesquisa do Hospital das Clínicas da Universidade de São Paulo, Certificado de Apresentação para Apreciação Ética de número 31063714.1.1001.0068, e foi inscrito no banco de ensaios clínicos clinicaltrials.gov sob o identificador NCT02535676. Todos os participantes leram e assinaram o Termo de Consentimento Livre e Esclarecido, assim como seus cuidadores quando aplicável. O estudo foi conduzido no Serviço Interdisciplinar de Neuromodulação

- Instituto de Psiquiatria do Hospital das Clínicas da Faculdade de Medicina da 
Universidade de São Paulo, sendo o período de recrutamento de Novembro de 2014 até Junho de 2017.

O recrutamento de sujeitos se deu por meio de divulgação em mídia (anúncios de rádio e jornal), assim como divulgação dentro do Instituto de Psiquiatria para possível encaminhamento de pacientes oriundos de outros serviços, em particular o Programa de Esquizofrenia do Instituto de Psiquiatria do HC-FMUSP (PROJESQ). Sujeitos interessados em participar do estudo entraram em contato com o serviço para agendamento de consulta de triagem, na qual foram verificados critérios de inclusão e exclusão. Os critérios de inclusão para o estudo foram:

- Diagnóstico de Esquizofrenia, segundo critérios do DSM-4-TR.

- Idade entre 18 e 60 anos.

- Pontuação na subescala de sintomas negativos da PANSS (Positive and negative syndrome scale - Escala de sintomas positivos e negativos) igual ou superior a 20.

Os critérios de exclusão foram:

- Uso de medicação antidepressiva.

- Uso de benzodiazepinicos em doses iguais ou superiores a 10mg de Diazepam, ou equivalente.

- Quadro clínico instável, por estar em planejamento terapêutico com necessidade de ajustes de medicamentos, ou por não aderência a propostas terapêuticas.

- Transtorno relacionado ao uso de álcool ou substâncias psicoativas.

- Diagnóstico de doenças orgânicas do sistema nervoso central, incluindo crises convulsivas, traumatismo crânio-encefálico ou histórico de intervenções neurocirúrgicas. 
- Tratamento com eletroconvulsoterapia nos últimos 6 meses.

- Presença de implantes metálicos ou dispositivos médicos eletrônicos em região cefálica (por exemplo implante coclear, estimulação cerebral profunda).

- Limiar motor acima de 80\% do valor total do aparelho de EMT.

A aplicação dos critérios de exclusão acima, assim como o método de aplicação do tratamento, seguiram protocolos de segurança estabelecidos pela Federação Internacional de Neurofisiologia Clínica para a aplicação de terapia biológica com estimulação cerebral não-invasiva (Antal et al., 2017; Rossi, Hallett, Rossini, Pascual-Leone, \& Safety of, 2009).

Os sujeitos incluídos foram randomizados em dois grupos, um grupo designado a receber a intervenção ativa e o outro a receber a intervenção placebo. A intervenção ativa se refere à estimulação com ETCC, e intervenção placebo se refere à estimulação sham, ambos detalhados a seguir. A randomização e a intervenção foram duplamente cegadas, de forma que nem os sujeitos tampouco os membros da equipe tinham conhecimento do tipo de tratamento (ativo ou placebo) que cada sujeito recebeu.

Antes do início do tratamento, os sujeitos foram submetidos a avaliação clínica, com coleta de dados demográficos e clínicos. Essa avaliação envolveu o preenchimento das escalas clínicas PANSS (Kay, Fiszbein, \& Opler, 1987), escala de alucinações auditivas (Bartels-Velthuis, van de Willige, Jenner, \& Wiersma, 2012), escala para avaliação de sintomas negativos (SANS) (Andreasen, 1989), escala de depressão de MontgomeryÅsberg (MADRS)(Montgomery \& Asberg, 1979). Os sujeitos foram reavaliados, com a repetição dessas escalas clínicas, de forma sequencial após a intervenção, sendo a 
avaliação e desfecho primário aquela referente a 6 semanas após a intervenção. A Tabela

2.1 expõe de forma sistemática os procedimentos de avaliação e intervenção realizados durante o ensaio clínico.

Os sujeitos foram também divididos em categorias. Foram identificados sujeitos como apresentando "alucinações refratárias" seguindo a definição de estudo prévio de ETCC em esquizofrenia, que selecionou sujeitos com alucinações diárias e contínuas, apesar de medicação antipsicótica (Brunelin et al., 2012).

TABELA 2.1 - Sequência de procedimentos realizados durante a participação no ensaio clínico STARTS

\begin{tabular}{|c|c|c|}
\hline Avaliação Inicial & $\begin{array}{l}\text { - Coleta de dados clínicos e } \\
\text { demográficos } \\
\text { - PANSS } \\
\text { - Escala de alucinações } \\
\text { auditivas } \\
\text { - SANS }\end{array}$ & $\begin{array}{l}\text { - } \text { MADRS } \\
\text { - } \mathbf{E C M}\end{array}$ \\
\hline $1^{\text {a }}$ semana de tratamento & \multicolumn{2}{|c|}{$\begin{array}{l}\text { Realização de } 2 \text { sessões diárias, durante } 5 \text { dias seguidos } \\
\text { ECM (apos a primeira estimulação por ETCC) }\end{array}$} \\
\hline $\begin{array}{l}\text { Após } 6 \text { semanas } \\
\text { *Avaliação de desfecho } \\
\text { primário }\end{array}$ & $\begin{array}{l}\text { Realização de } 1 \text { sessão de } \\
\text { manutenção } \\
\text { - PANSS } \\
\text { - Escala de alucinações } \\
\text { auditivas } \\
\text { - SANS }\end{array}$ & 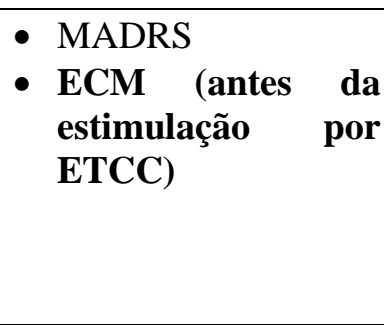 \\
\hline Após 12 semanas & $\begin{array}{l}\text { Realização de } 1 \text { sessão de } \\
\text { manutenção } \\
\text { - PANSS } \\
\text { - Escala de } \\
\text { auditivas } \\
\text { - SANS }\end{array}$ & 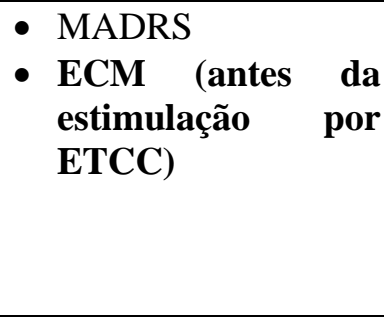 \\
\hline
\end{tabular}


As sessões de estimulação ocorreram ao longo de uma semana, sendo realizadas duas vezes ao dia por 5 dias consecutivos, totalizando 10 sessões. Cada sessão teve duração de 20 minutos.

\section{Aplicação da ETCC e cegamento}

A ETCC envolveu a utilização de dois eletrodos, de dimensões 5 x $7 \mathrm{~cm}$, posicionados sobre a superfície do couro cabeludo do paciente. Os eletrodos foram colocados dentro de uma esponja banhada em solução salina $(\mathrm{NaCl} 0.9 \%)$ que atua como condutor. O ânodo foi posicionado sobre a região correspondente ao córtex pré-frontal dorsolateral (CPFDL) esquerdo (posição F3 segundo o sistema internacional 10-20 de posicionamento de eletrodos de EEG), e o cátodo sobre a região correspondente ao córtex de associação auditiva, junção temporoparietal, situado no hemisfério esquerdo (posição média entre o T3 e o P3 segundo o sistema 10-20). O equipamento utilizado foi o $D C$ Stimulator (software for double-blinded studies) de marca Neuroconn.

A intensidade de corrente escolhida para o ensaio clínico foi $2 \mathrm{~mA}$. Observou-se que intensidades de corrente abaixo de $0.5 \mathrm{~mA}$ parecem não provocar efeitos neuromodulatórios consideráveis em sujeitos humanos, ao passo que intensidades acima de $2 \mathrm{~mA}$ provocam desconforto significativo (Nitsche \& Paulus, 2000). Utilizando intensidades de corrente dentro deste limite, Boggio et al. (Boggio et al., 2006) observaram que sessões de ETCC com intensidade de $2 \mathrm{~mA}$ comparadas a intensidade de $1 \mathrm{~mA}$ se associaram a melhor desempenho em tarefas de fluência verbal e memória operacional em sujeitos humanos, com estimulação anódica em região prefrontal. De fato, grande parte dos ensaios clínicos utilizaram intensidades de $2 \mathrm{~mA}$, demonstrando 
superioridade da estimulação em comparação com placebo no tratamento de condições clinicas como dor crônica (Fregni, Boggio, Lima, et al., 2006) e depressão maior (Fregni, Boggio, Nitsche, et al., 2006; Valiengo et al., 2017). Da mesma forma, ensaio clínico para tratamento de depressão que utilizou intensidade inferior, $1 \mathrm{~mA}$, não identificou diferença entre estimulação ativa e placebo (Loo et al., 2010).

Trabalho metodológico de preparação do ensaio clinico utilizou simulação computacional do campo elétrico induzido no córtex cerebral, quando aplicada a montagem descrita (Valiengo et al. no prelo). A simulação demonstrou pico de intensidade do campo elétrico na região dos córtices motor e sensorial primários do hemisfério esquerdo (Figura 2.1). Apesar desse pico em região cortical teoricamente não relevante para o tratamento em questão, a intensidade do campo elétrico no CPFDL esquerdo atingiu níveis considerados adequados para possível uso terapêutico no tratamento de pacientes com esquizofrenia (Brunoni et al., 2014). Não obstante, a presença de campo elétrico com intensidade suficiente para efeito neurofisiológico em córtex motor primário potencialmente levará a efeito sobre medidas de ECM, o que pode informar sobre a responsividade cortical dos participantes à sessão de ETCC (Rawji et al., 2018). 
FIGURA 2.1 - Simulação computacional do campo elétrico induzido por ETCC no córtex cerebral, utilizando de montagem descrita neste estudo. (extraído de Valiengo et al. no prelo)
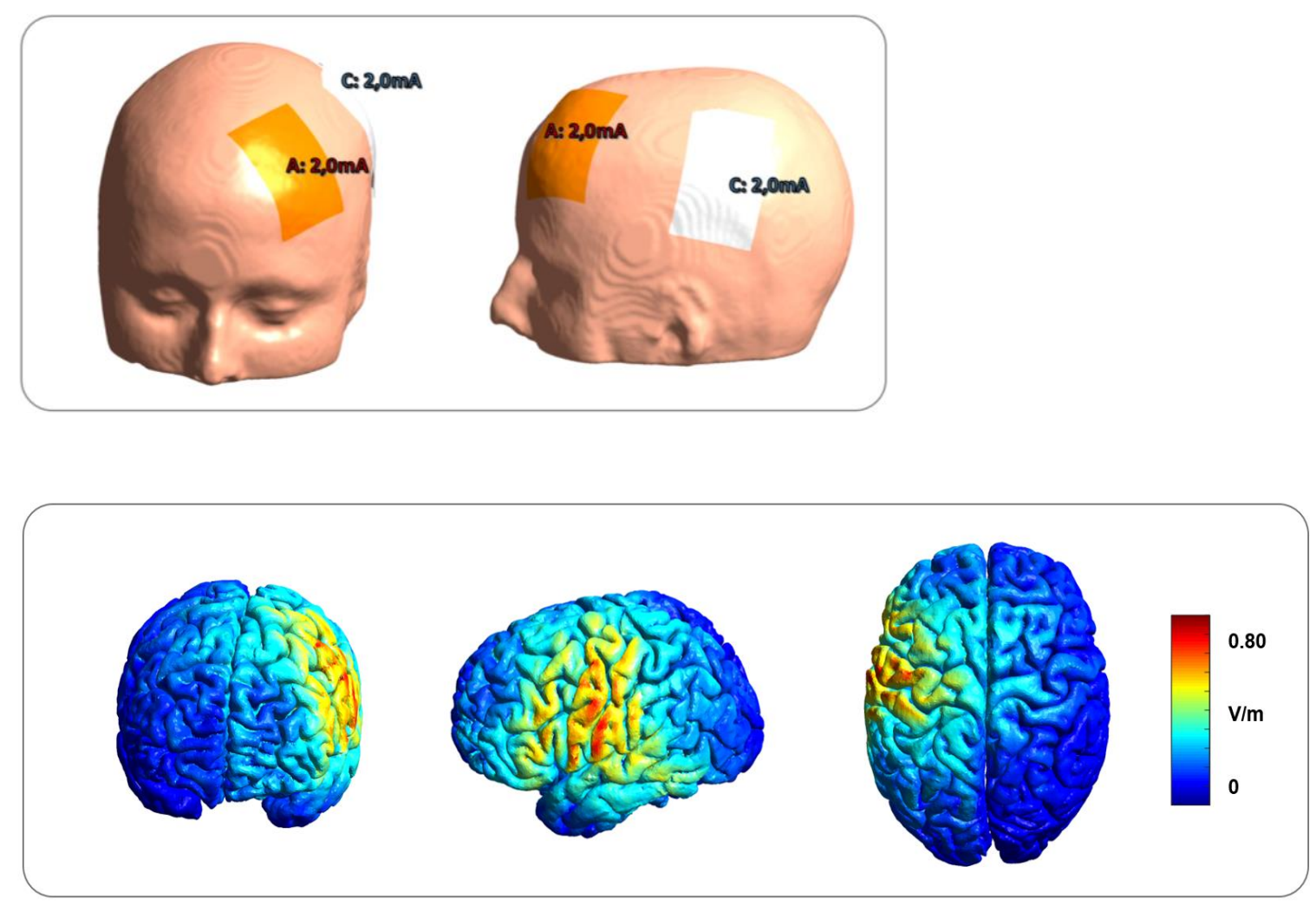

Acima, modelo representando a posição dos eletrodos de ETCC sobre o couro cabeludo. A: anodo; C: catodo.

Abaixo, modelo representando o resultado da simulação computacional do campo elétrico induzido no córtex cerebral, quando aplicada a montagem acima.

O aparelho também dispõe de uma função “estimulação sham". Diferentemente da estimulação ativa, na qual ocorre lento aumento da intensidade da corrente, mantendo-a em $2 \mathrm{~mA}$ durante a sessão, a estimulação sham envolve um lento aumento da corrente até $2 \mathrm{~mA}$ durante 15 segundos (suficiente para o sujeito se habituar à sensação de 
formigamento promovida pela estimulação) e em seguida cessa a passagem de corrente. Tal procedimento simula uma estimulação ativa, auxiliando a preservação do cegamento. Apesar de haver alguma passagem de corrente elétrica no início da sessão, esse procedimento não foi associado a qualquer efeito clínico relevante (Gandiga, Hummel, \& Cohen, 2006). O que estabelece se uma sessão é ativa ou sham é um código que deve ser digitado no aparelho logo antes da sessão. O aparelho dispõe de uma lista de códigos, cada um com 5 dígitos, que correspondem a estimulação ativa ou estimulação sham, que se encontra no manual de instrução do dispositivo. Antes do início do ensaio clínico, os códigos foram adicionados à lista de randomização, respeitando a natureza da intervenção da entrada na lista: ativo ou placebo. A correspondência de cada sujeito ao código em questão foi mantida em segredo do sujeito e da equipe durante o tratamento, de forma que tanto o sujeito quando o aplicador das sessões de ETCC e o avaliador estiveram cegados durante o tratamento e avaliações de seguimento de cada sujeito.

Cada sessão de ETCC se inicia com a colocação do sujeito confortavelemente em uma cadeira reclinável. A região cefálica do sujeito é medida e são marcados os locais alvos da estimulação, descritos acima. Os eletrodos do aparelho de ETCC são colocados dentro de esponjas de mesmas dimensões, banhadas com soro fisiológico, e em seguida colocadas em contato com o couro cabeludo do sujeito, nas regiões alvo marcadas. Os eletrodos são mantidos em posição com auxílio de bandas elásticas colocadas ao redor da região cefálica do sujeito. Em seguida o aparelho é ligado, de acordo com as especificações descritas acima. Durante a sessão o aplicador mantem-se nas proximidades do sujeito para garantir seu conforto e segurança. 


\section{Excitabilidade Cortical Motora por EMT}

A pesquisa da ECM foi realizada de acordo com recomendações de protocolos internacionais, (Chen et al., 2008; Groppa et al., 2012) e seguiu os procedimentos aplicados em estudo normativo de ECM (Cueva et al., 2016). O procedimento envolve a aplicação de um pulso magnético gerado por aparelho de EMT (marca e modelo "MagVenture X100", com 2 capacitores para protocolos de pulso pareado), utilizando uma bobina circular, na superfície do crânio correspondente córtex motor primário, especificamente a área do membro superior. A resposta motora foi mensurada por eletromiografia de superfície, colocando um eletrodo sobre o ventre do músculo alvo, músculo abductor pollicis brevis contralateral ao hemisfério estimulado, um eletrodo de referência sobre o tendão do mesmo músculo e um terceiro eletrodo terra em uma região neutra, distante dos anteriores.

Para a mensuração da ECM por pulso pareado, é necessário estabelecer o valor do limiar motor de repouso (LM), assim como as potências do aparelho necessárias para o "estimulo teste" e "estimulo condicionante", descritos a seguir. Os índices de ECM por pulso pareado são a razão da amplitude do PEM eliciado pelo pulso pareado sobre amplitude do PEM eliciado por um único estimulo não condicionado (ver sessão "INTRODUÇÃO", "Medidas de Excitabilidade Cortical utilizando Estimulação Magnética Transcraniana").

O LM foi mensurado, sendo definido como porcentagem mínima da carga total do capacitor da EMT capaz de eliciar potencial motor de $>50 \mu \mathrm{V}$, em ao menos 5 de 10 tentativas, tendo o músculo da mão relaxado (Chen et al., 2008; Rossini et al., 1999). Em seguida, foi pesquisada a intensidade capaz de produz resposta motora de amplitude média 
de $1 \mathrm{mV}$. Essa pesquisa se iniciou fixando a porcentagem do capacitor a $120 \%$ do LM, variando a intensidade até encontrar uma intensidade capaz de eliciar 5 PEM consecutivos de amplitude entre 0.5 e $1.5 \mathrm{mV}$. O valor encontrado foi considerado como a intensidade do "estimulo teste" para o pulso pareado. A amplitude do PEM do "estimulo teste" foi então medida 8 vezes antes e 8 vezes após os paradigmas de pulso pareado, sendo a média aritmética destes considerado o PEM por pulso único (estímulos testes não condicionados). Esse método de estabelecimento da intensidade do "estímulo teste" difere do estudo de normatização mencionado previamente, que utilizou o PEM eliciado por intensidade de 120\% do LM (Cueva et al., 2016), porém o procedimento aqui descrito foi o de escolha para a maioria dos estudos em transtornos neuropsiquiátricos em diferentes centros, especialmente em sujeitos com esquizofrenia, e foi assim escolhido para permitir futura comparação com tais estudos (Daskalakis, Christensen, Chen, et al., 2002; Hasan et al., 2012; Wobrock et al., 2008).

As variáveis referentes à excitabilidade cortical por pulso pareado serão estudadas através dos seguintes paradigmas:

1) Inibição intracortical de intervalo curto (SICI). Pesquisada com a aplicação de um pulso com potência de $80 \%$ do LM, considerado estímulo condicionante, seguido pelo "estímulo teste". São realizadas 2 medidas, com intervalo entre estímulos de 2 e $4 \mathrm{~ms}$ (SICI-2ms, SICI-4ms).

2) Facilitação intracortical (ICF). Pesquisada com a aplicação de um pulso com potência de $80 \%$ do LM, considerado estímulo condicionante, seguido pelo "estímulo teste”. São realizadas 2 medidas, com intervalo entre estímulos de 10 e $15 \mathrm{~ms}$ (ICF-10ms, ICF-15ms). 
Cada paradigma de intervalo entre estímulos de pulso pareado foi repetido 8 vezes, com intervalo de aproximadamente 3 segundos entre cada aplicação. $O$ dado final de cara paradigma de pulso pareado é a média aritmética da amplitude do PEM dos 8 testes. A ordem de aplicação dos paradigmas foi de forma não sequencial, para evitar possíveis efeitos da ordem de intervalos entre estímulos, embora a ordem tenha sido a mesma para todos os sujeitos. O resultado final de cada paradigma de pulso pareado é a razão entre a média aritmética da amplitude do PEM do do pulso pareado sobre a média aritmética da amplitude do potencial evocado pelo "estimulo teste não condicionado". SICI 2 e 4 ms, assim como ICF 10 e $15 \mathrm{~ms}$ foram agrupados pela média dessas medidas, obtendo-se assim apenas um valor para SICI e um valor para ICF (Bajbouj et al., 2006).

As medidas de excitabilidade cortical motora foram realizadas durante a avaliação inicial, fornecendo dados da linha de base de cada indivíduo, e imediatamente após a $1^{\text {a }}$ sessão de ETCC, fornecendo dados sobre a mudança da excitabilidade cortical motora em resposta a uma sessão. As medidas de excitabilidade cortical motora quando do desfecho primário (após 6 semanas) teve como objetivo verificar a mudança da linha de base dessa medida após o tratamento completo, em comparação com a medida precedendo o tratamento. Dessa forma, a medida foi realizada antes da sessão de manutenção, dado que a sessão de ETCC poderia provocar mudança adicional na excitabilidade cortical motora.

\subsection{Análise Estatística}

Inicialmente foi realizada uma caracterização da amostra utilizando estatística descritiva dos dados demográficos e clínicos, assim como dos dados obtidos pelos paradigmas de ECM. Os dados dessa variável habitualmente tem distribuição não normal, 
assim foram submetidos à transformação logarítmica de base exponencial $(e)$ - logaritmo natural. Optamos por esse procedimento, pois razões de variáveis biológicas potencialmente apresentam distribuição log-normal, cuja transformação logarítmica permite a utilização de testes paramétricos que presumem distribuição normal, e cujos cálculos de tamanho de efeito tem mais fácil interpretação. Para confirmação da característica da distribuição dessas variáveis, assim como avaliação de normalidade das demais variáveis, realizamos teste de hipótese de normalidade de Shapiro-Wilk. A comparação das variáveis clinicodemográficas e de ECM foram realizadas entre o grupo que recebeu tratamento ativo e o grupo que recebeu tratamento placebo, utilizando teste $\mathrm{t}$ para amostras independentes.

Análise da correlação entre medidas de ECM e variáveis clinicodemográficas envolveu modelos de regressão linear multivariável, utilizando método de eliminação backwards. Esse procedimento envolve a criação de uma sequência de modelos, sendo o primeiro um que contém todas as variáveis independentes de interesse (variáveis clinicodemográficas), em seguida a variável do modelo com menor significância estatística é removida, sendo em seguida realizado outro modelo, porem sem tal variável. Isso é repetido até que sobrem no modelo apenas variáveis estatisticamente significativas (limiar do p-valor < 0.05). Esse procedimento foi realizado para cada medida de ECM (SICI e ICF), que foram incluídas como variáveis dependentes. As variáveis independentes foram: Idade, gênero, preferência manual, duração de doença, anos de educação, dose equivalente de haloperidol (Andreasen, Pressler, Nopoulos, Miller, \& Ho, 2010), uso de clozapina, uso de benzodiazepínicos; e as escalas clínicas PANSS, a escala de alucinações auditivas, SANS, e MADRS. Devido à sobreposição de sintomas 
encontrados na PANSS e nas escalas restantes, e consequente risco de colinearidade, essas variáveis foram incluídas nos modelos separadamente. Aplicando correção de Bonferroni para múltiplas análises, foram considerados apenas resultados com significância p<0.0125.

Para avaliar os efeitos da ETCC sobre medidas de ECM, foram utilizados modelos lineares de efeitos mistos para medidas repetidas. Tal teste estatístico permite verificar se há diferenças significativas nas medidas repetidas em cada sujeito utilizando variáveis fixas: tempo (pré e pós intervenção), intervenção (placebo e ETCC ativa) e hemisfério no qual a ECM foi medida (esquerdo e direito). Dois modelos foram criados, tendo um o SICI e outro o ICF como variáveis dependentes. Como hipotetizamos que a mudança dos valores de ECM, entre pré e pós intervenção, ocorrerá apenas nos indivíduos que realizarem a intervenção ativa, incluímos a interação tempo*intervenção. Da mesma forma, hipotetizamos que tal mudança ocorrerá predominantemente no hemisférios que recebeu a ETCC, córtex esquerdo, e então incluímos a interação tempo* intervenção* hemisfério. Aplicando correção de Bonferroni para múltiplas análises, foram considerados apenas resultados com significância $\mathrm{p}<0.025$. 


\title{
3. RESULTADOS
}

\begin{abstract}
Amostra
No total, 450 indivíduos contataram o serviço via email, telefone, ou via referência medica para centro de especialidades. Destes, 216 compareceram para consulta de triagem e esclarecimento acerca do ensaio clínico. Apos avaliação de triagem, 116 indivíduos não preencheram critérios para a participação do ensaio clínico, ou recusaram a participação. Os 100 indivíduos restantes foram incluídos no ensaio clínico, sendo estes divididos em 2 grupos: tratamento ativo e tratamento placebo.
\end{abstract}


FIGURA 3.1 - Fluxograma da triagem e seleção de sujeitos para o ensaio clínico STARTS

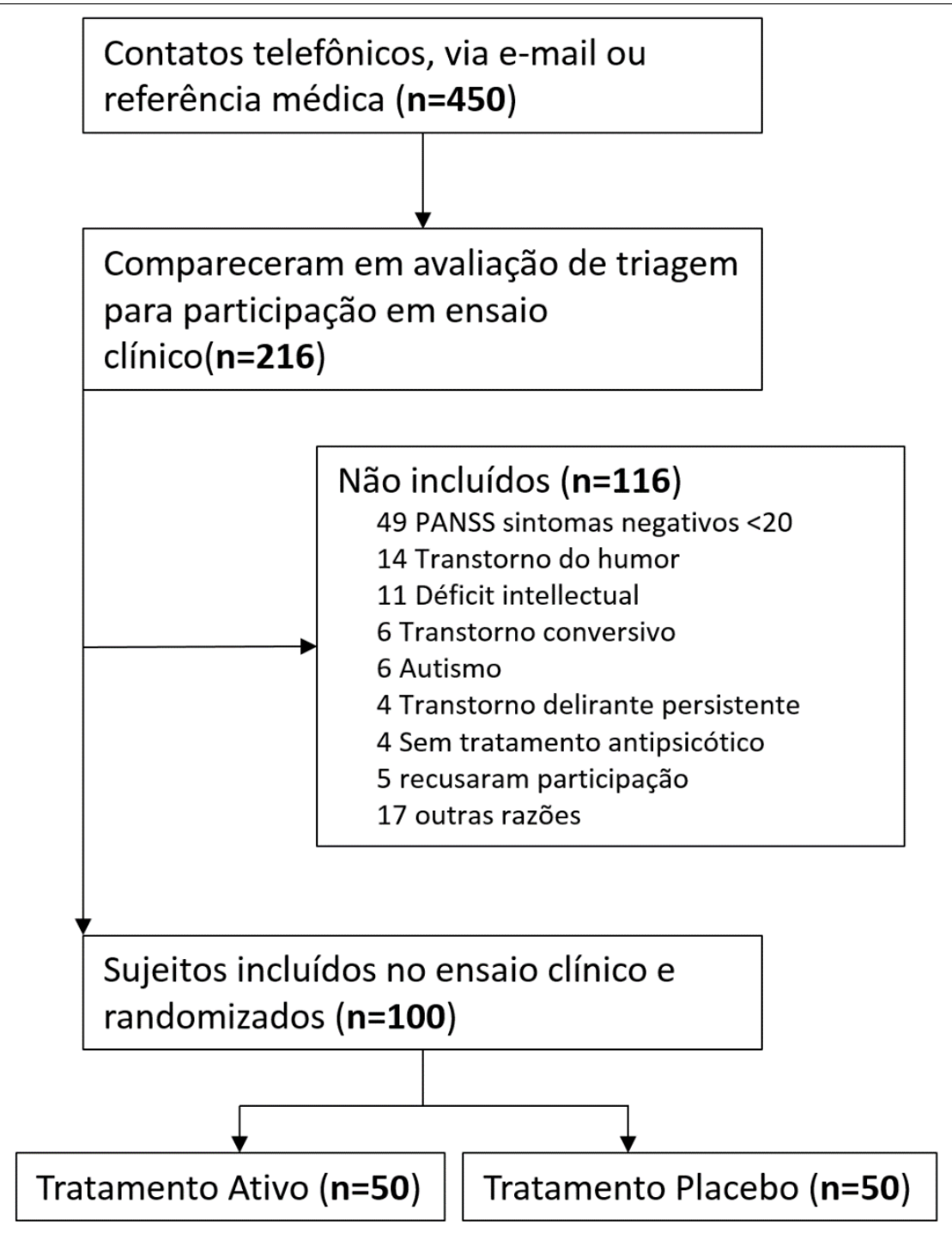

Dos 100 sujeitos incluídos no ensaio clínico, 90 compareceram para as medidas de ECM. Destes, 14 sujeitos apresentaram LM acima de $80 \%$ da potência total do aparelho, 4 apresentaram dados incompletos de medidas da ECM, 3 recusaram a realização do procedimento. Assim, 69 sujeitos contribuíram com dados de excitabilidade cortical 
motora para o presente estudo. Dados clínicos e demográficos da amostra final se encontram na tabela 3.1.

\section{TABELA 3.1 - Dados Clínicos e Demográficos, e de excitabilidade cortical motora de 69 sujeitos do ensaio clínico ETCC para sintomas negativos de esquizofrenia, e comparação dos grupos ativo e placebo}

\begin{tabular}{|c|c|c|c|c|}
\hline & \multirow{2}{*}{$\begin{array}{l}\text { TOTAL } \\
\mathrm{n}=69\end{array}$} & \multicolumn{2}{|c|}{ Randomização } & \multirow[b]{2}{*}{$\mathbf{p}$} \\
\hline & & $\begin{array}{l}\text { Ativo } \\
(n=23)\end{array}$ & $\begin{array}{l}\text { Placebo } \\
(n=25)\end{array}$ & \\
\hline \multicolumn{5}{|l|}{ Dados clinicodemográficos } \\
\hline Idade (anos - d.p.) & $35,2( \pm 8,5)$ & $33,2( \pm 7,1)$ & $35,8( \pm 10,1)$ & 0,299 \\
\hline Gênero (Homens - \%) & $56(81,2 \%)$ & $21(84 \%)$ & $20(87 \%)$ & 0,772 \\
\hline Destros (n - \%) & $57(92,1 \%)$ & $20(87 \%)$ & $24(96 \%)$ & 0,228 \\
\hline Duração da doença (anos - d.p.) & $13,9( \pm 7,6)$ & $12,7( \pm 9,0)$ & $13,3( \pm 6,7)$ & 0,800 \\
\hline Anos de educação (anos - d.p.) & $10,9( \pm 3,0)$ & $12,0( \pm 2,4)$ & $10,4( \pm 3,0)$ & 0,055 \\
\hline $\begin{array}{l}\text { Dose equivalente de Haloperidol (mg/dia - } \\
\text { d.p.) }\end{array}$ & $8,9( \pm 5,1)$ & $9,2( \pm 4,6)$ & $7,6( \pm 4,8)$ & 0,255 \\
\hline Usa Clozapina ( $n$ - \%) & $46(66,6 \%)$ & $15(65,2 \%)$ & $19(76,0 \%)$ & 0,412 \\
\hline Usa Benzodiazepínico (n - \%) & $6(7,9 \%)$ & $0(0 \%)$ & $2(8 \%)$ & 0,182 \\
\hline PANSS-TOTAL (escore - d.p.) & $71,2( \pm 11,4)$ & $70,5( \pm 13,8)$ & $70,3( \pm 10,9)$ & 0,956 \\
\hline - $\quad$ PANSS-Positivo & $14,0( \pm 4,0)$ & $13,7( \pm 4,2)$ & $13,1( \pm 3,7)$ & 0,611 \\
\hline - $\quad$ PANSS-Negativo† & $24,8( \pm 3,6)$ & $24,8( \pm 4,3)$ & $25,0( \pm 3,5)$ & 0,876 \\
\hline - $\quad$ PANSS-Geral & $32,3( \pm 6,8)$ & $32,0( \pm 8,8)$ & $32,2( \pm 6,1)$ & 0,927 \\
\hline SANS (score - d.p.) & $61,0( \pm 12,4)$ & $60,8( \pm 14,3)$ & $63,0( \pm 10,4)$ & 0,532 \\
\hline AVHRS (score - d.p.) † & $9,1( \pm 12,4)$ & $8,6( \pm 12,6)$ & $6,4( \pm 11,5)$ & 0,537 \\
\hline - $\quad$ Alucinações refratárias $(n-\%)$ & $27(39,1 \%)$ & $8(34,8 \%)$ & $7(28,0 \%)$ & 0,613 \\
\hline MADRS (escore - d.p.) & $10,5( \pm 6,7)$ & $10,3( \pm 7,1)$ & $10,6( \pm 6,5)$ & 0,901 \\
\hline \multicolumn{5}{|l|}{ Excitabilidade Cortical motora } \\
\hline SICI direito (index - d.p.) † & $-0,66( \pm 0,86)$ & $-0,40( \pm 0,86)$ & $-0,65( \pm 0,92)$ & 0,611 \\
\hline ICF direito (index - d.p.) † & $-0,05( \pm 0,64)$ & $-0,01( \pm 0,80)$ & $-0,03( \pm 0,59)$ & 0,961 \\
\hline SICl esquerdo (index - d.p.) † & $-0,50( \pm 0,98)$ & $-0,23( \pm 1,31)$ & $-0,48( \pm 0,88)$ & 0,601 \\
\hline ICF esquerdo (index - d.p.) † & $0,15\left( \pm 0,61^{\prime}\right)$ & $0,09( \pm 0,69)$ & $0,14( \pm 0,68)$ & 0,955 \\
\hline $\begin{array}{l}\text { Resultados reportados como media } \pm \text { desvio } \\
\text { (n) e percentagem para variáveis binarias. } \\
\dagger \text { variáveis analisadas utilizando dados apos t }\end{array}$ & Irão (d.p.) $\mathrm{p}$ & & & \\
\hline
\end{tabular}


O teste de normalidade de Shapiro-Wilk descartou a hipótese de normalidade da distribuição dos valores das escalas PANSS-Negativo escala de alucinações auditivas e dos valores dos índices de ECM $(\mathrm{p}<0.001)$. Os valores dessas variáveis foram então submetidos a transformação logarítmica e então testados novamente. Teste dos dados variáveis após transformação logarítmica não descartou hipótese de normalidade. Análises estatísticas dessas variáveis foram realizadas com os dados após transformação logarítmica.

\section{Correlação dados de excitabilidade cortical e variáveis clínicas}

Observando os resultados dos modelos de regressão linear incluindo todas as variáveis de interesse, encontramos nível elevado de colinearidade (medido pelo fator de inflação de covariância) nas variáveis relativas às escalas clinicas (TABELAS 3.2, 3.3, 3.4 e 3.5), justificando a inclusão destas no modelo de forma separada, como foi descrito na sessão 2.4 (Análise Estatística). 
TABELA 3.2 - Modelo de regressão linear multivariável incluindo todas as variáveis independentes no modelo, tendo como variável dependente a excitabilidade cortical motora (SICI em córtex esquerdo) em amostra de 69 sujeitos com esquizofrenia

\begin{tabular}{lccccccc}
\hline SICI & B & $\begin{array}{l}\text { Erro } \\
\text { padrão }\end{array}$ & $\boldsymbol{\beta}$ & $\mathbf{t}$ & $\mathbf{p}$ & $\begin{array}{l}\text { Fator de inflação } \\
\text { de covariância }\end{array}$ \\
\hline Gênero & 0,217 & 0,243 & 0,119 & 0,893 & 0,377 & 1,299 \\
Idade & $-0,038$ & 0,014 & $-0,479$ & $-2,617$ & 0,012 & 2,435 \\
Anos de educação & $-0,004$ & 0,029 & $-0,018$ & $-0,146$ & 0,885 & 1,119 \\
Duração da doença & 0,003 & 0,017 & 0,037 & 0,195 & 0,846 & 2,661 \\
Dextro & $-0,614$ & 0,390 & $-0,217$ & $-1,574$ & 0,122 & 1,383 \\
Dose equvalente de & 0,030 & 0,021 & 0,218 & 1,466 & 0,149 & 1,601 \\
haloperidol & & & & & & \\
Usa clozapina & 0,135 & 0,197 & 0,091 & 0,689 & 0,494 & 1,265 \\
Usa benzodiazepínico & $-0,559$ & 0,316 & $-0,219$ & $-1,772$ & 0,083 & 1,113 \\
PANSS-Positivo & $-0,022$ & 0,032 & $-0,126$ & $-0,669$ & 0,507 & 2,565 \\
PANSS-Negativo & 0,050 & 0,043 & 0,258 & 1,149 & 0,257 & 3,673 \\
PANSS-Geral & 0,032 & 0,019 & 0,319 & 1,645 & 0,107 & 2,731 \\
AVHRS & $-0,005$ & 0,010 & $-0,083$ & $-0,477$ & 0,635 & 2,215 \\
MADRS & $-0,003$ & 0,016 & $-0,032$ & $-0,206$ & 0,838 & 1,708 \\
SANS & $-0,021$ & 0,014 & $-0,354$ & $-1,502$ & 0,140 & 4,042 \\
\hline
\end{tabular}


TABELA 3.3 - Modelo de regressão linear multivariável incluindo todas as variáveis independentes no modelo, tendo como variável dependente a excitabilidade cortical motora (SICI em córtex direito) em amostra de 69 sujeitos com esquizofrenia

\begin{tabular}{lccccccc}
\hline SICI & B & $\begin{array}{l}\text { Erro } \\
\text { Córtex direito }\end{array}$ & $\boldsymbol{\beta}$ & $\mathbf{t}$ & $\mathbf{p}$ & $\begin{array}{l}\text { Fator de inflação } \\
\text { de covariância }\end{array}$ \\
\hline Gênero & 0,138 & 0,267 & 0,070 & 0,518 & 0,607 & 1,297 \\
Idade & $-0,016$ & 0,016 & $-0,189$ & $-1,022$ & 0,312 & 2,438 \\
Anos de educação & 0,024 & 0,031 & 0,095 & 0,777 & 0,441 & 1,081 \\
Duração da doença & $-0,013$ & 0,019 & $-0,129$ & $-0,658$ & 0,514 & 2,734 \\
Dextro & $-1,114$ & 0,386 & $-0,400$ & $-2,885$ & 0,006 & 1,377 \\
Dose equvalente de & & & & & & \\
haloperidol & 0,055 & 0,023 & 0,369 & 2,411 & 0,020 & 1,677 \\
Usa clozapina & $-0,059$ & 0,214 & $-0,037$ & $-0,277$ & 0,783 & 1,261 \\
Usa benzodiazepínico & $-0,401$ & 0,345 & $-0,144$ & $-1,160$ & 0,252 & 1,102 \\
PANSS-Positivo & $-0,034$ & 0,035 & $-0,180$ & $-0,949$ & 0,347 & 2,586 \\
PANSS-Negativo & 0,029 & 0,047 & 0,141 & 0,622 & 0,537 & 3,706 \\
PANSS-Geral & 0,007 & 0,021 & 0,064 & 0,328 & 0,744 & 2,701 \\
AVHRS & 0,001 & 0,011 & 0,013 & 0,070 & 0,944 & 2,323 \\
MADRS & $-0,017$ & 0,017 & $-0,148$ & $-0,967$ & 0,338 & 1,670 \\
SANS & 0,000 & 0,015 & $-0,007$ & $-0,028$ & 0,978 & 3,996 \\
\hline
\end{tabular}


TABELA 3.4 - Modelo de regressão linear multivariável incluindo todas as variáveis independentes no modelo, tendo como variável dependente a excitabilidade cortical motora (ICF em córtex esquerdo) em amostra de 69 sujeitos com esquizofrenia

\begin{tabular}{lrrrrrrc}
\hline ICF & B & $\begin{array}{l}\text { Erro } \\
\text { Córtex esquerdo }\end{array}$ & $\boldsymbol{\beta}$ & $\mathbf{t}$ & $\mathbf{p}$ & $\begin{array}{l}\text { Fator de inflação } \\
\text { de covariância }\end{array}$ \\
\hline Gênero & 0,174 & 0,195 & 0,127 & 0,892 & 0,377 & 1,181 \\
Idade & $-0,007$ & 0,013 & $-0,102$ & $-0,503$ & 0,617 & 2,422 \\
Anos de educação & 0,019 & 0,026 & 0,101 & 0,715 & 0,478 & 1,169 \\
Duração da doença & 0,003 & 0,016 & 0,035 & 0,162 & 0,872 & 2,772 \\
Dextro & 0,112 & 0,311 & 0,054 & 0,359 & 0,721 & 1,335 \\
Dose equvalente de & & & & & & \\
haloperidol & 0,006 & 0,017 & 0,060 & 0,382 & 0,704 & 1,461 \\
Usa clozapina & 0,116 & 0,172 & 0,100 & 0,674 & 0,504 & 1,278 \\
Usa benzodiazepínico & $-0,249$ & 0,283 & $-0,121$ & $-0,882$ & 0,382 & 1,106 \\
PANSS-Positivo & 0,018 & 0,029 & 0,127 & 0,608 & 0,546 & 2,550 \\
PANSS-Negativo & $-0,009$ & 0,036 & $-0,058$ & $-0,255$ & 0,800 & 3,009 \\
PANSS-Geral & 0,010 & 0,016 & 0,120 & 0,617 & 0,540 & 2,207 \\
AVHRS & $-0,017$ & 0,009 & $-0,376$ & $-1,987$ & 0,052 & 2,098 \\
MADRS & 0,007 & 0,013 & 0,080 & 0,501 & 0,618 & 1,480 \\
SANS & $-0,005$ & 0,011 & $-0,100$ & $-0,442$ & 0,660 & 3,018 \\
\hline
\end{tabular}




\section{TABELA 3.5 - Modelo de regressão linear multivariável incluindo todas as variáveis independentes no modelo, tendo como variável dependente a excitabilidade cortical motora (ICF em córtex direito) em amostra de 69 sujeitos com esquizofrenia}

\begin{tabular}{|c|c|c|c|c|c|c|}
\hline $\begin{array}{l}\text { ICF } \\
\text { Córtex direito }\end{array}$ & B & $\begin{array}{l}\text { Erro } \\
\text { padrão }\end{array}$ & $\boldsymbol{\beta}$ & $\mathbf{t}$ & $\mathbf{p}$ & $\begin{array}{l}\text { Fator de inflação } \\
\text { de covariância }\end{array}$ \\
\hline Gênero & 0,292 & 0,234 & 0,170 & 1,250 & 0,217 & 1,180 \\
\hline Idade & 0,015 & 0,015 & 0,187 & 0,960 & 0,342 & 2,411 \\
\hline Anos de educação & $-0,012$ & 0,030 & $-0,052$ & $-0,395$ & 0,694 & 1,107 \\
\hline Duração da doença & $-0,023$ & 0,019 & $-0,264$ & $-1,253$ & 0,216 & 2,833 \\
\hline Dextro & 0,025 & 0,341 & 0,010 & 0,073 & 0,942 & 1,307 \\
\hline $\begin{array}{l}\text { Dose equvalente de } \\
\text { haloperidol }\end{array}$ & 0,003 & 0,020 & 0,025 & 0,163 & 0,871 & 1,486 \\
\hline Usa clozapina & 0,348 & 0,205 & 0,241 & 1,697 & 0,096 & 1,285 \\
\hline Usa benzodiazepínico & $-0,178$ & 0,338 & $-0,069$ & $-0,525$ & 0,602 & 1,088 \\
\hline PANSS-Positivo & 0,031 & 0,034 & 0,186 & 0,920 & 0,362 & 2,615 \\
\hline PANSS-Negativo & $-0,033$ & 0,040 & $-0,174$ & $-0,839$ & 0,405 & 2,747 \\
\hline PANSS-Geral & $-0,026$ & 0,019 & $-0,262$ & $-1,391$ & 0,170 & 2,253 \\
\hline AVHRS & $-0,014$ & 0,010 & $-0,265$ & $-1,404$ & 0,166 & 2,271 \\
\hline MADRS & 0,003 & 0,016 & 0,030 & 0,198 & 0,844 & 1,480 \\
\hline SANS & 0,008 & 0,012 & 0,135 & 0,632 & 0,530 & 2,911 \\
\hline
\end{tabular}

Observando o resultado do modelo de regressão linear após o procedimento de eliminação backwards, apenas o valor de SICI no hemisfério esquerdo foi significativamente correlacionado com idade, com maiores valores do índice encontrados em sujeitos de maior idade, correspondendo a menor inibição intracortical (TABELA 3.6). Nenhuma outra variável alcançou significância para inclusão no modelo. Nenhum modelo significativo foi encontrado correlacionando ICF com variáveis clinicodemograficas. 
TABELA 3.6 - Modelo de regressão linear multivariável com resultados significativos, incluindo apenas variáveis clinicodemograficas significativamente correlacionadas com índices de excitabilidade cortical motora, em amostra de 69 sujeitos com esquizofrenia

\begin{tabular}{lccccccc}
\hline & $\mathbf{B}$ & $\begin{array}{c}\text { Erro } \\
\text { padrão }\end{array}$ & $\boldsymbol{\beta}$ & $\mathbf{t}$ & $\mathbf{p}$ & $\mathbf{R}^{\mathbf{2}}$ \\
\hline $\begin{array}{l}\text { SICI } \\
\text { Córtex esquerdo }\end{array}$ & Idade & 0.044 & 0.014 & 0.366 & 3.146 & 0.003 & 0.120 \\
\hline
\end{tabular}

\section{Análise do impacto das intervenções na excitabilidade cortical motora}

Medidas da ECM imediatamente após a primeira sessão de ETCC foram realizadas em 48 sujeitos. Houve diferença significativa da inibição intracortical no hemisfério esquerdo, demonstrado pelo modelo linear de efeitos mixtos de medidas repetidas, apenas significativo quando avaliado pela interação tempo* intervenção* hemisfério, descrito na TABELA 3.7. Na FIGURA 3.2 podemos observar que a mudança se deu pela diminuição dos índices de SICI após a intervenção, especialmente no hemisferio esquerdo, naqueles individuos que realizaram a intervenção ativa, traduzindo aumento da inibição intracortical. Uma tendência semelhante ocorreu na analise do ICF, observado na FIGURA 3.2, embora não tenha alcançado significância estatística. 
TABELA 3.7 - Modelo linear de efeitos mixtos de medidas repetidas, com variáveis fixas tempo (pré e pós intervenção), intervenção (placebo e ETCC ativa) e hemisfério de medida da ECM (esquerdo e direito), tendo índices de ECM como variáveis independentes (modelo $1 \mathrm{com}$, SICI, modelo $2 \mathrm{com}$ ICF) em amostra de 69 sujeitos com esquizofrenia

\begin{tabular}{lllllll}
\hline & Estimado & Erro & t & df & p \\
& & & padrão & & \\
\hline SICI & intervenção & 0.052 & 0.154 & 0.343 & 230 & 0.731 \\
& tempo & 0.119 & 0.112 & 1.061 & 230 & 0.289 \\
& hemisfério & 0.077 & 0.085 & 0.899 & 230 & 0.369 \\
& tempo*intervenção & -0.084 & 0.189 & -0.447 & 230 & 0.654 \\
& tempo*intervenção*hemisfério & -0.457 & 0.194 & -2.350 & 230 & $\mathbf{0 . 0 1 9}$ \\
\hline ICF & intervenção & 0.004 & 0.115 & 0.035 & 230 & 0.971 \\
& tempo & 0.143 & 0.102 & 1.407 & 230 & 0.160 \\
& hemisfério & 0.144 & 0.079 & 1.814 & 230 & 0.070 \\
& tempo*intervenção & -0.049 & 0.171 & -0.288 & 230 & 0.773 \\
& tempo*intervenção*hemisfério & -0.195 & 0.177 & -1.102 & 230 & 0.271 \\
\hline
\end{tabular}


FIGURA 3.2 - ECM por pulso pareado em amostra de 69 sujeitos com esquizofrenia antes e após sessão de ETCC, divididos em intervenção ativa e intervenção placebo, e medidas realizadas no córtex cerebral direito e esquedo.
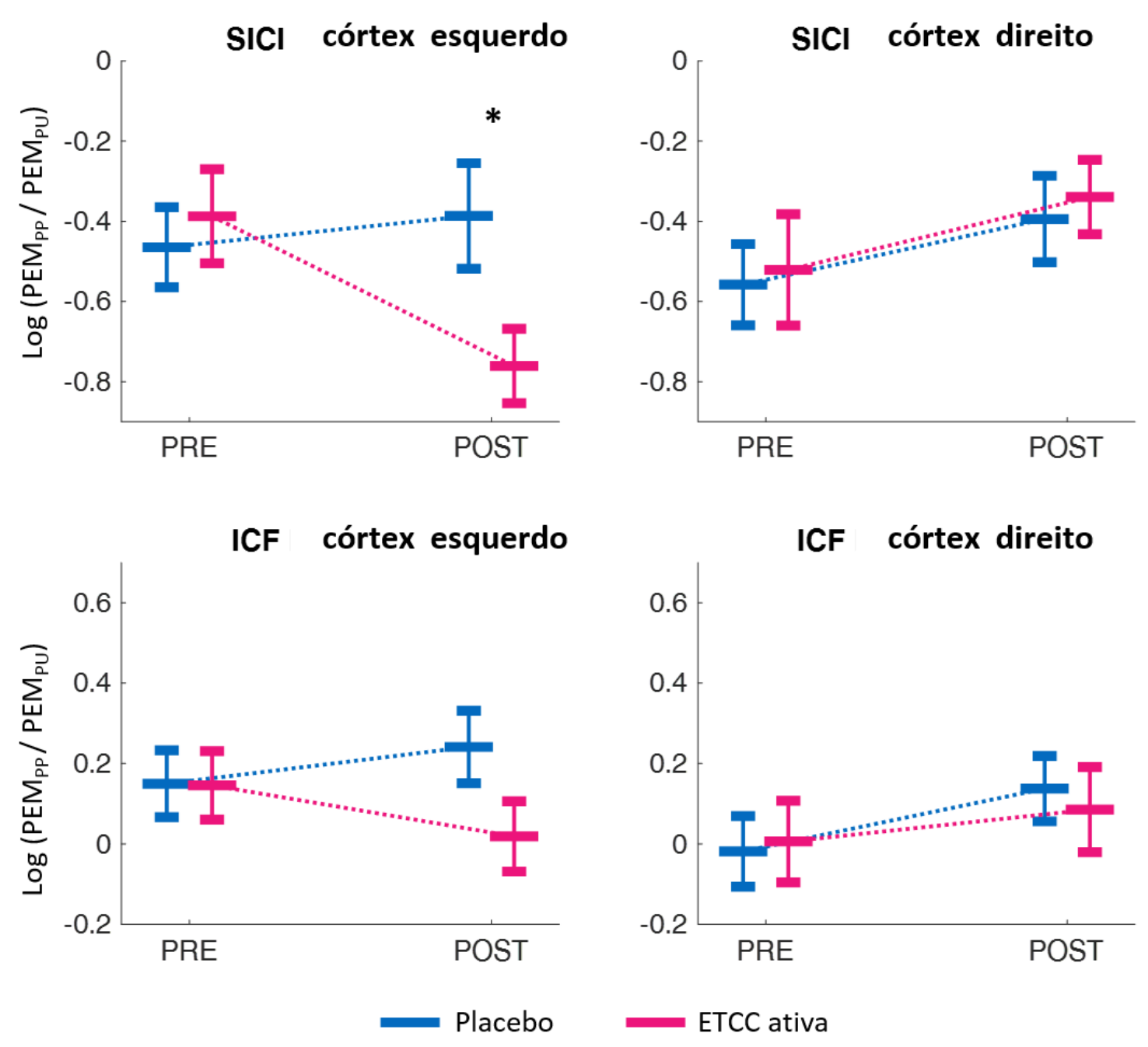

\footnotetext{
Resultado de ECM por pulso pareado descrito como logaritmo da razão da amplitude do PEM eliciado por pulso pareado (PP), com intervalos entre estímulos de 2, 4 ms (agrupados como SICI), e 10 e 15 ms (agrupados como ICF); sobre amplitude do PEM eliciado por pulso único (PU). $* \mathrm{p}<0.025$
} 


\section{CONCLUSÕES}

Os objetivos do presente estudo incluíram a avaliação dos padrões de excitabilidade cortical motora em uma população de sujeitos com esquizofrenia que participaram de ensaio clínico, este tendo como intervenção a realização de ETCC como estratégica terapêutica para sintomas negativos. Também se propôs avaliar a mudança de padrões de excitabilidade cortical motora após a realização de sessão de ETCC, e, por fim, a investigação de padrões de excitabilidade cortical motora, como possíveis preditores de resposta clínica ao tratamento proposto.

Os dados obtidos permitiram a realização de análises e obtenção de resultados que endereçaram às duas primeiras questões, como será discutido a seguir. Entretanto, até o momento da redação do presente trabalho, não foi possível contar com os dados de desfecho do ensaio clínico em questão, pois estes estão sendo avaliados e processados para publicação. Dessa forma, não foi possível a realização de análises para responder à terceira questão proposta. Uma vez analisados os dados do desfecho clínico e publicados os resultados do ensaio clínico STARTS, será então possível comparar as medidas de ECM com a resposta terapêutica dos sujeitos.

\section{Excitabilidade cortical motora em sujeitos com esquizofrenia}

A comparação de padrões de excitabilidade cortical motora entre sujeitos com esquizofrenia e indivíduos saudáveis já fora testada em estudos prévios. Apesar de achado recente descrevendo aumento de SICI em pacientes com esquizofrenia não medicados (Schecklmann, Weidler, Eichhammer, Hajak, \& Langguth, 2018), o conjunto de resultados 
prévios convergem para diminuição de SICI em portadores de esquizofrenia comparados a sujeitos saudáveis (Radhu et al., 2013), incluindo estudos que investigaram especificamente pacientes não medicados (Daskalakis, Christensen, Chen, et al., 2002; Wobrock et al., 2008), representando déficit da inibição intracortical nessa população. A relevância de tal achado envolve o fato de corroborar com a hipótese da presença de déficit de inibição intracortical em pacientes com esquizofrenia já proposta por outras investigações utilizando ferramentas de neuroimagem e genética, além de fortalecer a existência de uma ferramenta neurofisiologica adicional capaz de avaliar esse déficit.

Os resultados do presente estudo sugerem a existência de algumas variáveis associadas a diferentes padrões de ECM na amostra de sujeitos com esquizofrenia. Encontramos correlação entre idade e SICI no hemisfério cerebral esquerdo, sugerindo diminuição da inibição intracortical em sujeitos de mais idade. A associação entre níveis de SICI e idade avançada já fora observada em amostras de sujeitos saudáveis (Cueva et al., 2016; Peinemann, Lehner, Conrad, \& Siebner, 2001). Tais achados realizados por meio de ECM investigada por TMS estão de acordo com estudos que revelam diminuição de inibição intracortical em populações de mais idade por outros meios, por exemplo, evidenciando diminuição da concentração de GABA em córtex cerebral (Gao et al., 2013; McQuail, Frazier, \& Bizon, 2015).

Não foram encontradas correlações entre medidas de excitabilidade cortical e conjuntos de sintomas característicos de esquizofrenia no presente estudo. Algumas características da amostra estudada podem ter contribuído para o resultado negativo. Inicialmente pode-se argumentar que todos os sujeitos do ensaio clínico encontravam-se 
medicados com fármacos de ação em sistema nervoso central, mais especificamente medicações antipsicóticas, e, portanto, passíveis de interferência nas medidas de excitabilidade cortical. Contrapondo essa interpretação, um estudo envolveu a administração de medicações antipsicóticas (típicos e atípicos) em sujeitos saudáveis, não sendo observado qualquer impacto significativo destas nas medidas de ECM (Daskalakis et al., 2003). Entretanto os autores mencionam como limitação o fato de que sujeitos em uso crônico dessas medicações podem apresentar alterações que tal estudo em não pode testar. De fato, observou-se que sujeitos com esquizofrenia não medicados apresentam menores índices de inibição intracortical comparados com aqueles medicados (Liu, Fitzgerald, Daigle, Chen, \& Daskalakis, 2009), assim como foi observado o aumento da inibição intracortical em pacientes que passaram a utilizar Clozapina continuamente (Kaster et al., 2015). Tal achado pode ser interpretado de duas formas: indivíduos que usam esses fármacos apresentam alteração do padrão de inibição intracortical devido a efeito farmacologico das substâncias, apenas evidente com o uso crônico; ou, aumento da inibição intracortical com o uso desses fármacos se torna evidente apenas em pacientes com esquizofrenia, população que apresenta déficit de inibição intracortical, possivelmente representando uma "normalização" da ECM. Entretanto, não foi possível identificar tal diferença de efeito no presente estudo, dada a homogeneidade da amostra, que incluiu apenas sujeitos que se encontravam em tratamento crônico.

De fato, a homogeneidade da presente amostra provavelmente está implicada nos resultados negativos deste estudo. Devido ao fato da amostra corresponder àquela do ensaio clínico no qual o presente estudo foi incluído, esta representa uma subpopulação específica de sujeitos portadores de esquizofrenia, como explícito pelos critérios de 
inclusão: doença de longa duração (duração média de doença de mais 14 anos), em tratamento de longo prazo, com sintomas positivos estáveis (média da amostra PANSS sintomas positivos de 14), porém com sintomas negativos proeminentes (média da amostra na PANSS sintomas negativos de 25). Tal seleção é ideal para a condução do ensaio clinico, desenhado para estudar especificamente a evolução de sintomas negativos com a intervenção neste grupo de doentes. Entretanto, devido à variabilidade reduzida de variáveis clínicas, em comparação com a totalidade dos indivíduos portadores de esquizofrenia, não foi possível descartar a hipótese nula de correlações entre ECM e variáveis clínicas.

\section{Mudança da excitabilidade cortical motora em sujeitos com esquizofrenia após} sessão de ETCC

O presente estudo também teve como objetivo a avaliação da mudança de excitabilidade cortical após sessão de ETCC. Alterações da amplitude do PEM seguido de intervenção com potencial efeito neuromodulador tem sido utilizadas como medida indireta de neuroplasticidade (Hassanzahraee, Zoghi, \& Jaberzadeh, 2018; Ziemann et al., 2008). De fato, efeitos neuroplásticos da ETCC foram primeiramente investigados com diferenças de amplitude do MEP (Nitsche \& Paulus, 2000). O uso de paradigmas de pulso pareado, além de parecerem mais sensíveis a alterações neuroplásticas provocadas por intervenções (Ziemann et al., 2008), sugerem mecanismos neurofisiológicos específicos pelos quais a eventual modulação ocorre, com alterações do SICI decorrendo de circuitaria GABAérgica de interneurônios rápidos (Di Lazzaro, Oliviero, et al., 2005), e alterações do 
ICF decorrendo em grande medida de interneurônios excitatórios glutamatérgicos (Di Lazzaro et al., 2006; Ziemann et al., 2015).

Em estudo prévio, Hasan e colaboradores (Hasan et al., 2013) utilizaram ETCC para estimulação da área motora primária em sujeitos com esquizofrenia. O estudo concluiu que as sessões de ETCC não foram capazes de alterar a ECM desses sujeitos de forma significativa, mensurada pela razão do PEM, sugerindo que pacientes com esquizofrenia apresentam déficit da plasticidade cortical, e, portanto, apresentariam resposta insatisfatória à ETCC. Os resultados que obtivemos contradizem em parte essa conclusão, dado que encontramos diferenças entre os sujeitos que realizaram estimulação ativa e estimulação placebo, com significativo aumento do SICI. Diferentes resultados podem ter ocorrido devido a diferentes medidas de plasticidade, pois como mencionado acima, sugere-se que medidas por paradigmas de pulso pareado sejam mais sensíveis a mudanças neuroplásticas comparados a mudanças da amplitude absoluta do PEM (Ziemann et al., 2008). Outra diferença se deve a intensidades de corrente distintas utilizadas na ETCC, $1 \mathrm{~mA}$ em contraposição ao presente estudo, no qual utilizamos $2 \mathrm{~mA}$. É sabido que o simples aumento de intensidade de corrente de ETCC não resulta em alteração linear de respostas neurofisiológicas (Batsikadze, Moliadze, Paulus, Kuo, \& Nitsche, 2013; Jamil et al., 2017). Entretanto, mudanças na densidade da corrente utilizada tem efeito sobre a mudança de excitabilidade cortical, com maiores densidades levando a efeito mais robusto (Kuo et al., 2013; Nitsche et al., 2007). Tal efeito também foi observado em ETCC dirigida ao córtex pré-frontal (Gordon et al., 2018; Hill, Rogasch, Fitzgerald, \& Hoy, 2017), sugerindo que maiores densidades de corrente aplicadas sobre o córtex pré-frontal apresentam maior eficácia no que diz respeito a efeitos neuromodulatórios. 
Tal argumentação levanta a questão de como ETCC em regiões corticais distantes do córtex motor primário pode ter levado a efeito sobre ECM. Primeiramente, efeitos significativos sobre a ECM foram observados não apenas com a colocação de eletrodos diretamente na área correspondente ao córtex motor primário, mas também com montagens de eletrodos dispostos distantes dessa região, porém mantendo-o entre os eletrodos.(Rawji et al., 2018). De fato, o uso de simulação computacional permitiu a visualização do campo elétrico induzido pela presente montagem de ETCC, revelando pico de intensidade sobre região que inclui o córtex motor primário esquerdo, no mesmo hemisfério em que resultados significativos de SICI foram encontrados (FIGURA 2.1).

Acerca do impacto da polaridade da ETCC sobre parâmetros de ECM avaliada com pulsos pareados, estudos anteriores reunidos em meta-análise sugerem que ETCC de polaridade anódica diminui o SICI, e resultados são inconclusivos para estimulação catódica (Biabani et al., 2018). Entretanto, como mencionado acima, no presente estudo o posicionamento dos eletrodos foi distante do córtex motor primário, não sendo possível uma comparação direta com estudos que estimularam especificamente tal região cortical. Ademais, é possível que efeitos da ETCC em indução de neuroplasticidade sejam diferentes entre sujeitos saudáveis e sujeitos com esquizofrenia. De fato, sujeitos com esquizofrenia apresentam déficit na indução de neuromodulação, sendo demonstrado por respostas deficitárias ao protocolo de estimulação associativa pareada (paired associative stimulation protocol - PAS), medida associada ao fenômeno de plasticidade LTP, dependente de glutamato. (Frantseva et al., 2008; Hasan et al., 2011; Strube et al., 2016). É provável que tal déficit de neuromodulação seja responsável pela ineficiência da ETCC na modulação do ICF, fenômeno dependente de atividade glutamatérgica, explicando a 
ausência de alteração dessa medida de ECM no presente estudo, mas mantendo efeito sobre SICI. Ademais, a presente amostra encontra-se particularmente susceptível a esse déficit, dado que dificuldade de indução de LTP é particularmente pronunciada em indivíduos com forma de doença mais avançada (Hasan et al., 2011).

A presença de aumento significativo do SICI sugere assim efeito neuromodulatório em outra via, mais especificamente ação no sentido de aumento da atividade de interneurônios GABA $A_{A}$ (Di Lazzaro, Oliviero, et al., 2005). Curiosamente, estudos prévios utilizando ETCC anódica sobre o CPFDL e mensurando resposta neuromodulatória com EMT-EEG demonstraram efeito condizente com aumento da inibição intracortical, por exemplo, com diminuição da magnitude de oscilações corticais de banda gama (Gordon et al., 2018; Hill et al., 2017). Ambos SICI e oscilações corticais de banda gama tem o mesmo mecanismo em comum, mais especificamente a atividade sincrônica de interneurônios $\mathrm{GABA}_{\mathrm{A}}$ (Lisman \& Jensen, 2013). É possível que o efeito principal de estimulação anódica sobre o CDLPF seja no sentido de facilitação da atividade da circuitaria GABAergica, diminuindo a magnitude de oscilações gama e o PEM de pulsos pareados de intervalo curto entre pulsos.

Alterações da circuitaria interneuronal de sujeitos com esquizofrenia também foram identificadas de outras formas. Mutações genéticas envolvendo moléculas relacionadas à síntese de GABA e seus receptores foram identificadas como fator de risco para o desenvolvimento de esquizofrenia (Akbarian \& Huang, 2006). Modelos matemáticos e modelos animais demonstram que a atividade oscilatória organizada de interneurônios intracorticais inibitórios GABAergicos tem importância fundamental na atividade cortical, 
sendo diretamente implicados na atividade tônica dos neurônios piramidais. De fato, a atividade dessa circuitaria está correlacionada com respostas piramidais de maior amplitude, e consequente processamento e transmissão adequados de informação neuronal. (Hasenstaub et al., 2005; Hu, Gan, \& Jonas, 2014; Lisman \& Jensen, 2013). Dessa forma, o funcionamento anormal de rede interneuronal GABAérgica pode ser responsável por diversos achados em sujeitos com esquizofrenia. Déficit GABAérgico pode ser responsável por falha do funcionamento cortical adequado, levando à hipoatividade em córtex pré-frontal observado em sujeitos com esquizofrenia (Minzenberg et al., 2009), que ocorre concomitantemente com marcadores de desinibição intracortical, como déficit de SICI (Radhu et al., 2013). Por fim, déficit GABAégico pode estar associado a resposta terapêutica observada com medicações agonistas GABAérgicos, como benzodiazepínicos e Clozapina, assim como o aumento de SICI com a administração desta última (Kaster et al., 2015; Liu et al., 2009).

Curiosamente, também observamos aumento do SICI após a sessão de ETCC. É possível que o efeito dessa estimulação nesse grupo de sujeitos seja a regulação da circuitaria neuronal GABAérgica, trazendo-a para níveis mais "normais", e, possivelmente, exercendo poder terapêutico. Tais efeitos podem ser responsáveis pela melhora de sintomas cognitivos e alucinatórios observados em estudos prévios (Mondino et al., 2016; Smith et al., 2015), assim como eventuais melhoras de sintomas negativos a serem observadas nos resultados do presente ensaio clínico. Entretanto, tal hipótese deve ser confirmada futuramente com a comparação desse fenômeno com mensuração objetiva de respostas à terapia. 


\subsection{Limitações}

As limitações relevantes do presente estudo serão discutidas a seguir.

Como mencionado, o desenho do presente estudo, sendo incluído dentro de um ensaio clínico, é inadequado para responder questões acerca da comparação de marcadores de ECM com diferentes variáveis clinicodemográficas. Tais questões podem ser melhor respondidas em estudo exploratório envolvendo mensuração de ECM em uma amostra numerosa, porém heterogênea de pacientes com esquizofrenia, no qual os participantes apresentem diferentes características clinicodemograficas, incluindo níveis de gravidade diferentes em diferentes domínios. Outra possibilidade seria um estudo transversal dirigido a uma hipótese especifica, correlacionando medidas de excitabilidade cortical motora entre pacientes com esquizofrenia que diferem em uma característica alvo a ser estudada (p.ex. ECM em sujeitos com esquizofrenia com diferentes níveis de sintomas positivos, tempos de duração de doença etc) porém pareados com relação as outras variáveis clinicodemográficas, especialmente no caso da idade como fator de confusão para tempo de duração de doença.

Acerca da mensuração de efeitos neuromodulatórios seguidos de sessão de ETCC, pode-se criticar o uso de medida de ECM para a avaliação de estimulação de região cortical não motora. Afinal, ECM pode apenas ser investigada em região que apresenta PEM, e técnicas mais recentes combinando EMT e EEG teoricamente permitem a exploração de respostas corticais em qualquer região capaz de gerar sinais de EEG 
(Ilmoniemi \& Kicic, 2010; Rogasch \& Fitzgerald, 2013). Entretanto, algumas limitações desse argumento devem ser colocadas em questão. Primeiramente, as técnicas necessárias para a combinação de EMT e EEG, assim como a interpretação de resultados, são principalmente compreendidas para o córtex motor, sendo outras regiões corticais ainda pouco estudadas (Hill et al., 2016). As dinâmicas da ECM, ao contrário, têm sido estudadas há mais tempo, de forma que os resultados encontrados em estudo com a técnica são de mais fácil interpretação e correspondência com mecanismos neurofisiológicos. Ademais, apesar do principal alvo da ETCC no presente estudo não ter sido o córtex motor primário, como demonstrado em sessão anterior, tal região apresentou pico de intensidade do campo elétrico induzido pela estimulação, o que permitiu modulação da excitabilidade cortical observada por meio de ECM (Rawji et al., 2018). Por fim, os resultados encontrados no presente estudo, especificamente estimulação anódica em CPFDL levando ao aumento da inibição intracortical devido a aumento do SICI, corresponde a achados prévios em sujeitos saudáveis, analisados por meio de EMT EEG (Gordon et al., 2018; Hill et al., 2017).

Finalmente, não foi possível no presente momento responder à questão relativa à correlação do aumento da inibição intracortical com evolução do quadro clínico em resposta a terapia com ETCC, dada a indisponibilidade dos dados quando da formulação desse documento. 


\section{SUGESTÕES PARA TRABALHOS FUTUROS}

Primeiramente será de grande importância a correlação dos achados de neuromodulação cortical mencionados anteriormente com a evolução clínica em resposta à terapia. Com a conclusão e publicação do ensaio clínico STARTS, poderemos avaliar a resposta clínica dos participantes e comparar com medidas de ECM. Caso encontremos correlação entre evolução favorável de sintomas negativos com aumento da inibição intracortical, teremos evidências de que o mecanismo de ação desse protocolo de ETCC no sentido de aumentar a atividade inibitória intracortical está de fato envolvido na redução dos sintomas negativos, neuromodulando atividade cortical interneuronal GABAérgica deficitária nessa população de sujeitos.

Tal desenvolvimento significará o possível uso dessas medidas de excitabilidade cortical como biomarcador em esquizofrenia, refletindo disfunção de circuitaria interneuronal GABAérgica, e cuja neuromodulação levaria a resposta clínica favorável. O uso desse biomarcador pode ser utilizado como preditor de resposta, possivelmente identificando sujeitos que apresentam formas da doença com maior déficit nessa rede neuronal e que irão se beneficiar de tratamentos direcionados especificamente a neuromodulação desse circuito. A identificação de subgrupos de sujeitos portadores de esquizofrenia que apresentem esse padrão de déficit de excitabilidade cortical pode contribuir para um diagnóstico mais preciso de pacientes na pratica clínica, auxiliando no direcionamento de terapêuticas mais eficazes para cada indivíduo. Tais desenvolvimentos podem se dar em conjunto com o uso de medidas mais refinadas de excitabilidade cortical, possivelmente envolvendo técnicas mais direcionadas à região de interesse, como o uso de 
EMT-EEG; que, além de fornecer mais informações sobre a atividade cortical da região estudada, podem apresentar maior sensibilidade na identificação de mudanças da excitabilidade cortical.

Por fim, o uso dessas medidas como biomarcardores pode auxiliar no desenvolvimento de estratégias terapêuticas mais eficazes, como no futuro desenvolvimento de técnicas de intervenção por neuromodulação não invasiva (EMT, ETCC ou outras). Por exemplo, ao invés de conduzir um ensaio clínico para cada mudança de protocolo, pode-se optar por experimentar diferentes protocolos na eficácia sobre a modulação da excitabilidade cortical, e então conduzir um único ensaio clínico para aquele protocolo que fornecer mudanças mais exuberantes e consistentes no marcador alvo. O uso de tal estratégia no estudo de terapias para sintomas de esquizofrenia pode reduzir significativamente o tempo e os custos para o encontro de tratamentos de maior eficácia para a doença. 


\section{REFERÊNCIAS BIBLIOGRÁFICAS}

Akbarian, S., \& Huang, H. S. (2006). Molecular and cellular mechanisms of altered GAD1/GAD67 expression in schizophrenia and related disorders. Brain Res Rev, 52(2), 293-304. doi:10.1016/j.brainresrev.2006.04.001

Andreasen, N. C. (1989). The Scale for the Assessment of Negative Symptoms (SANS): conceptual and theoretical foundations. Br J Psychiatry Suppl(7), 49-58.

Andreasen, N. C., Pressler, M., Nopoulos, P., Miller, D., \& Ho, B. C. (2010). Antipsychotic dose equivalents and dose-years: a standardized method for comparing exposure to different drugs. Biol Psychiatry, 67(3), 255-262. doi:10.1016/j.biopsych.2009.08.040

Antal, A., Alekseichuk, I., Bikson, M., Brockmoller, J., Brunoni, A. R., Chen, R., .. . Paulus, W. (2017). Low intensity transcranial electric stimulation: Safety, ethical, legal regulatory and application guidelines. Clin Neurophysiol, 128(9), 1774-1809. doi:10.1016/j.clinph.2017.06.001

Bajbouj, M., Lisanby, S. H., Lang, U. E., Danker-Hopfe, H., Heuser, I., \& Neu, P. (2006). Evidence for impaired cortical inhibition in patients with unipolar major depression. Biol Psychiatry, 59(5), 395-400. doi:10.1016/j.biopsych.2005.07.036

Barker, A. T., Jalinous, R., \& Freeston, I. L. (1985). Non-invasive magnetic stimulation of human motor cortex. Lancet, 1(8437), 1106-1107.

Barr, M. S., Farzan, F., Tran, L. C., Fitzgerald, P. B., \& Daskalakis, Z. J. (2012). A randomized controlled trial of sequentially bilateral prefrontal cortex repetitive transcranial magnetic stimulation in the treatment of negative symptoms in schizophrenia. Brain Stimul, 5(3), 337-346. doi:10.1016/j.brs.2011.06.003

Bartels-Velthuis, A. A., van de Willige, G., Jenner, J. A., \& Wiersma, D. (2012). Consistency and reliability of the auditory vocal hallucination rating scale $\begin{array}{llll}\text { (AVHRS). Epidemiol } \quad \text { Psychiatr } & \text { Sci, 21(3), }\end{array}$ doi:10.1017/S2045796012000108

Batsikadze, G., Moliadze, V., Paulus, W., Kuo, M. F., \& Nitsche, M. A. (2013). Partially non-linear stimulation intensity-dependent effects of direct current stimulation on motor cortex excitability in humans. $J$ Physiol, 591(7), 1987-2000. doi:10.1113/jphysiol.2012.249730

Biabani, M., Aminitehrani, M., Zoghi, M., Farrell, M., Egan, G., \& Jaberzadeh, S. (2018). The effects of transcranial direct current stimulation on short-interval intracortical inhibition and intracortical facilitation: a systematic review and meta-analysis. Rev Neurosci, 29(1), 99-114. doi:10.1515/revneuro-2017-0023

Boggio, P. S., Ferrucci, R., Rigonatti, S. P., Covre, P., Nitsche, M., Pascual-Leone, A., \& Fregni, F. (2006). Effects of transcranial direct current stimulation on working 
memory in patients with Parkinson's disease. J Neurol Sci, 249(1), 31-38. doi:10.1016/j.jns.2006.05.062

Brenner, H. D., Dencker, S. J., Goldstein, M. J., Hubbard, J. W., Keegan, D. L., Kruger, G., . . Midha, K. K. (1990). Defining treatment refractoriness in schizophrenia. Schizophr Bull, 16(4), 551-561.

Brunelin, J., Mondino, M., Gassab, L., Haesebaert, F., Gaha, L., Suaud-Chagny, M. F., .. . Poulet, E. (2012). Examining transcranial direct-current stimulation (tDCS) as a treatment for hallucinations in schizophrenia. Am J Psychiatry, 169(7), 719-724. doi:10.1176/appi.ajp.2012.11071091

Brunoni, A. R., Moffa, A. H., Sampaio-Junior, B., Borrione, L., Moreno, M. L., Fernandes, R. A., . . . Investigators, E.-T. (2017). Trial of Electrical Direct-Current Therapy versus Escitalopram for Depression. $N$ Engl J Med, 376(26), 2523-2533. doi:10.1056/NEJMoa1612999

Brunoni, A. R., Nitsche, M. A., Bolognini, N., Bikson, M., Wagner, T., Merabet, L., .. . Fregni, F. (2012). Clinical research with transcranial direct current stimulation (tDCS): challenges and future directions. Brain Stimul, 5(3), 175-195. doi:10.1016/j.brs.2011.03.002

Brunoni, A. R., Shiozawa, P., Truong, D., Javitt, D. C., Elkis, H., Fregni, F., \& Bikson, M. (2014). Understanding tDCS effects in schizophrenia: a systematic review of clinical data and an integrated computation modeling analysis. Expert Rev Med Devices, 11(4), 383-394. doi:10.1586/17434440.2014.911082

Brunoni, A. R., Valiengo, L., Baccaro, A., Zanao, T. A., de Oliveira, J. F., Goulart, A., . . . Fregni, F. (2013). The sertraline vs. electrical current therapy for treating depression clinical study: results from a factorial, randomized, controlled trial. JAMA Psychiatry, 70(4), 383-391. doi:10.1001/2013.jamapsychiatry.32

Buckley, P., Miller, A., Olsen, J., Garver, D., Miller, D. D., \& Csernansky, J. (2001). When symptoms persist: clozapine augmentation strategies. Schizophr Bull, 27(4), 615-628.

Burke, D., Hicks, R., Stephen, J., Woodforth, I., \& Crawford, M. (1995). Trial-to-trial variability of corticospinal volleys in human subjects. Electroencephalogr Clin Neurophysiol, 97(5), 231-237.

Chen, R., Cros, D., Curra, A., Di Lazzaro, V., Lefaucheur, J. P., Magistris, M. R., . . . Ziemann, U. (2008). The clinical diagnostic utility of transcranial magnetic stimulation: report of an IFCN committee. Clin Neurophysiol, 119(3), 504-532. doi:10.1016/j.clinph.2007.10.014

Chen, R., Tam, A., Butefisch, C., Corwell, B., Ziemann, U., Rothwell, J. C., \& Cohen, L. G. (1998). Intracortical inhibition and facilitation in different representations of the human motor cortex. J Neurophysiol, 80(6), 2870-2881. doi:10.1152/jn. 1998.80.6.2870 
Chong, H. Y., Teoh, S. L., Wu, D. B., Kotirum, S., Chiou, C. F., \& Chaiyakunapruk, N. (2016). Global economic burden of schizophrenia: a systematic review. Neuropsychiatr Dis Treat, 12, 357-373. doi:10.2147/NDT.S96649

Cueva, A. S., Galhardoni, R., Cury, R. G., Parravano, D. C., Correa, G., Araujo, H., .. . Ciampi de Andrade, D. (2016). Normative data of cortical excitability measurements obtained by transcranial magnetic stimulation in healthy subjects. Neurophysiol Clin, 46(1), 43-51. doi:10.1016/j.neucli.2015.12.003

Daskalakis, Z. J., Christensen, B. K., Chen, R., Fitzgerald, P. B., Zipursky, R. B., \& Kapur, S. (2002). Evidence for impaired cortical inhibition in schizophrenia using transcranial magnetic stimulation. Arch Gen Psychiatry, 59(4), 347-354.

Daskalakis, Z. J., Christensen, B. K., Chen, R., Fitzgerald, P. B., Zipursky, R. B., \& Kapur, S. (2003). Effect of antipsychotics on cortical inhibition using transcranial magnetic stimulation. Psychopharmacology (Berl), 170(3), 255-262. doi:10.1007/s00213003-1548-1

Daskalakis, Z. J., Christensen, B. K., Fitzgerald, P. B., \& Chen, R. (2002). Transcranial magnetic stimulation: a new investigational and treatment tool in psychiatry. $J$ Neuropsychiatry Clin Neurosci, 14(4), 406-415. doi:10.1176/jnp.14.4.406

Daskalakis, Z. J., Farzan, F., Barr, M. S., Maller, J. J., Chen, R., \& Fitzgerald, P. B. (2008). Long-interval cortical inhibition from the dorsolateral prefrontal cortex: a TMSEEG study. Neuropsychopharmacology, 33(12), 2860-2869. doi:10.1038/npp.2008.22

Daskalakis, Z. J., Farzan, F., Radhu, N., \& Fitzgerald, P. B. (2012). Combined transcranial magnetic stimulation and electroencephalography: its past, present and future. Brain Res, 1463, 93-107. doi:10.1016/j.brainres.2012.04.045

Dayan, E., Censor, N., Buch, E. R., Sandrini, M., \& Cohen, L. G. (2013). Noninvasive brain stimulation: from physiology to network dynamics and back. Nat Neurosci, 16(7), 838-844. doi:10.1038/nn.3422

Di Lazzaro, V., Oliviero, A., Pilato, F., Saturno, E., Dileone, M., Mazzone, P., . . . Rothwell, J. C. (2004). The physiological basis of transcranial motor cortex stimulation in conscious humans. Clin Neurophysiol, 115(2), 255-266.

Di Lazzaro, V., Oliviero, A., Saturno, E., Dileone, M., Pilato, F., Nardone, R., . . Tonali, P. (2005). Effects of lorazepam on short latency afferent inhibition and short latency intracortical inhibition in humans. $J$ Physiol, 564(Pt 2), 661-668. doi:10.1113/jphysiol.2004.061747

Di Lazzaro, V., Pilato, F., Dileone, M., Tonali, P. A., \& Ziemann, U. (2005). Dissociated effects of diazepam and lorazepam on short-latency afferent inhibition. $J$ Physiol, 569(Pt 1), 315-323. doi:10.1113/jphysiol.2005.092155

Di Lazzaro, V., Pilato, F., Oliviero, A., Dileone, M., Saturno, E., Mazzone, P., . . . Rothwell, J. C. (2006). Origin of facilitation of motor-evoked potentials after paired magnetic stimulation: direct recording of epidural activity in conscious humans. $J$ Neurophysiol, 96(4), 1765-1771. doi:10.1152/jn.00360.2006 
Di Lazzaro, V., Rothwell, J. C., Oliviero, A., Profice, P., Insola, A., Mazzone, P., \& Tonali, P. (1999). Intracortical origin of the short latency facilitation produced by pairs of threshold magnetic stimuli applied to human motor cortex. Exp Brain Res, 129(4), 494-499.

Farzan, F., Barr, M. S., Hoppenbrouwers, S. S., Fitzgerald, P. B., Chen, R., Pascual-Leone, A., \& Daskalakis, Z. J. (2013). The EEG correlates of the TMS-induced EMG silent period in humans. Neuroimage, 83, 120-134. doi:10.1016/j.neuroimage.2013.06.059

Fervaha, G., Foussias, G., Agid, O., \& Remington, G. (2014). Impact of primary negative symptoms on functional outcomes in schizophrenia. Eur Psychiatry, 29(7), 449455. doi:10.1016/j.eurpsy.2014.01.007

Frantseva, M. V., Fitzgerald, P. B., Chen, R., Moller, B., Daigle, M., \& Daskalakis, Z. J. (2008). Evidence for impaired long-term potentiation in schizophrenia and its relationship to motor skill learning. Cereb Cortex, 18(5), 990-996. doi:10.1093/cercor/bhm151

Freedman, R. (2003). Schizophrenia. $N$ Engl J Med, 349(18), 1738-1749. doi:10.1056/NEJMra035458

Fregni, F., Boggio, P. S., Lima, M. C., Ferreira, M. J., Wagner, T., Rigonatti, S. P., . . . Pascual-Leone, A. (2006). A sham-controlled, phase II trial of transcranial direct current stimulation for the treatment of central pain in traumatic spinal cord injury. Pain, 122(1-2), 197-209. doi:10.1016/j.pain.2006.02.023

Fregni, F., Boggio, P. S., Nitsche, M. A., Marcolin, M. A., Rigonatti, S. P., \& PascualLeone, A. (2006). Treatment of major depression with transcranial direct current stimulation. Bipolar Disord, 8(2), 203-204. doi:10.1111/j.1399-5618.2006.00291.x

Frohlich, F., Burrello, T. N., Mellin, J. M., Cordle, A. L., Lustenberger, C. M., Gilmore, J. H., \& Jarskog, L. F. (2016). Exploratory study of once-daily transcranial direct current stimulation (tDCS) as a treatment for auditory hallucinations in schizophrenia. Eur Psychiatry, 33, 54-60. doi:10.1016/j.eurpsy.2015.11.005

Gandiga, P. C., Hummel, F. C., \& Cohen, L. G. (2006). Transcranial DC stimulation (tDCS): a tool for double-blind sham-controlled clinical studies in brain stimulation. Clin Neurophysiol, 117(4), 845-850. doi:10.1016/j.clinph.2005.12.003

Gao, F., Edden, R. A., Li, M., Puts, N. A., Wang, G., Liu, C., . . . Barker, P. B. (2013). Edited magnetic resonance spectroscopy detects an age-related decline in brain GABA levels. Neuroimage, 78, 75-82. doi:10.1016/j.neuroimage.2013.04.012

Gilio, F., Curra, A., Lorenzano, C., Modugno, N., Manfredi, M., \& Berardelli, A. (2000). Effects of botulinum toxin type A on intracortical inhibition in patients with dystonia. Ann Neurol, 48(1), 20-26.

Gordon, P. C., Zrenner, C., Desideri, D., Belardinelli, P., Zrenner, B., Brunoni, A. R., \& Ziemann, U. (2018). Modulation of cortical responses by transcranial direct current stimulation of dorsolateral prefrontal cortex: A resting-state EEG and TMS-EEG study. Brain Stimul. doi:10.1016/j.brs.2018.06.004 
Groppa, S., Oliviero, A., Eisen, A., Quartarone, A., Cohen, L. G., Mall, V., . . Siebner, H. R. (2012). A practical guide to diagnostic transcranial magnetic stimulation: report of an IFCN committee. Clin Neurophysiol, 123(5), 858-882. doi:10.1016/j.clinph.2012.01.010

Hanajima, R., Ugawa, Y., Terao, Y., Enomoto, H., Shiio, Y., Mochizuki, H., . . . Kanazawa, I. (2002). Mechanisms of intracortical I-wave facilitation elicited with paired-pulse magnetic stimulation in humans. J Physiol, 538(Pt 1), 253-261.

Hanajima, R., Ugawa, Y., Terao, Y., Furubayashi, T., Machii, K., Shiio, Y., . . Kanazawa, I. (1999). Intracortical inhibition of the motor cortex is normal in chorea. J Neurol Neurosurg Psychiatry, 66(6), 783-786.

Hanajima, R., Ugawa, Y., Terao, Y., Sakai, K., Furubayashi, T., Machii, K., . . . Kanazawa, I. (1998). Cortico-cortical inhibition of the motor cortical area projecting to sternocleidomastoid muscle in normals and patients with spasmodic torticollis or essential tremor. Electroencephalogr Clin Neurophysiol, 109(5), 391396.

Hasan, A., Bergener, T., Nitsche, M. A., Strube, W., Bunse, T., Falkai, P., \& Wobrock, T. (2013). Impairments of motor-cortex responses to unilateral and bilateral direct current stimulation in schizophrenia. Front Psychiatry, 4, 121. doi:10.3389/fpsyt.2013.00121

Hasan, A., Nitsche, M. A., Rein, B., Schneider-Axmann, T., Guse, B., Gruber, O., . . . Wobrock, T. (2011). Dysfunctional long-term potentiation-like plasticity in schizophrenia revealed by transcranial direct current stimulation. Behav Brain Res, 224(1), 15-22. doi:10.1016/j.bbr.2011.05.017

Hasan, A., Wobrock, T., Grefkes, C., Labusga, M., Levold, K., Schneider-Axmann, T., .. . Bechdolf, A. (2012). Deficient inhibitory cortical networks in antipsychotic-naive subjects at risk of developing first-episode psychosis and first-episode schizophrenia patients: a cross-sectional study. Biol Psychiatry, 72(9), 744-751. doi:10.1016/j.biopsych.2012.03.005

Hasenstaub, A., Shu, Y., Haider, B., Kraushaar, U., Duque, A., \& McCormick, D. A. (2005). Inhibitory postsynaptic potentials carry synchronized frequency information in active cortical networks. Neuron, 47(3), 423-435. doi:10.1016/j.neuron.2005.06.016

Hassanzahraee, M., Zoghi, M., \& Jaberzadeh, S. (2018). How different priming stimulations affect the corticospinal excitability induced by noninvasive brain stimulation techniques: a systematic review and meta-analysis. Rev Neurosci. doi:10.1515/revneuro-2017-0111

Hill, A. T., Rogasch, N. C., Fitzgerald, P. B., \& Hoy, K. E. (2016). TMS-EEG: A window into the neurophysiological effects of transcranial electrical stimulation in nonmotor brain regions. Neurosci Biobehav Rev, 64, 175-184. doi:10.1016/j.neubiorev.2016.03.006 
Hill, A. T., Rogasch, N. C., Fitzgerald, P. B., \& Hoy, K. E. (2017). Effects of prefrontal bipolar and high-definition transcranial direct current stimulation on cortical reactivity and working memory in healthy adults. Neuroimage, 152, 142-157. doi:10.1016/j.neuroimage.2017.03.001

Hu, H., Gan, J., \& Jonas, P. (2014). Interneurons. Fast-spiking, parvalbumin(+) GABAergic interneurons: from cellular design to microcircuit function. Science, 345(6196), 1255263. doi:10.1126/science.1255263

Ilmoniemi, R. J., \& Kicic, D. (2010). Methodology for combined TMS and EEG. Brain Topogr, 22(4), 233-248. doi:10.1007/s10548-009-0123-4

Jamil, A., Batsikadze, G., Kuo, H. I., Labruna, L., Hasan, A., Paulus, W., \& Nitsche, M. A. (2017). Systematic evaluation of the impact of stimulation intensity on neuroplastic after-effects induced by transcranial direct current stimulation. J Physiol, 595(4), 1273-1288. doi:10.1113/JP272738

Kaster, T. S., de Jesus, D., Radhu, N., Farzan, F., Blumberger, D. M., Rajji, T. K., . . . Daskalakis, Z. J. (2015). Clozapine potentiation of GABA mediated cortical inhibition in treatment resistant schizophrenia. Schizophr Res, 165(2-3), 157-162. doi:10.1016/j.schres.2015.04.015

Kay, S. R., Fiszbein, A., \& Opler, L. A. (1987). The positive and negative syndrome scale (PANSS) for schizophrenia. Schizophr Bull, 13(2), 261-276.

Kossev, A. R., Siggelkow, S., Dengler, R., \& Rollnik, J. D. (2003). Intracortical inhibition and facilitation in paired-pulse transcranial magnetic stimulation: effect of conditioning stimulus intensity on sizes and latencies of motor evoked potentials. $J$ Clin Neurophysiol, 20(1), 54-58.

Kujirai, T., Caramia, M. D., Rothwell, J. C., Day, B. L., Thompson, P. D., Ferbert, A., . . Marsden, C. D. (1993). Corticocortical inhibition in human motor cortex. J Physiol, 471, 501-519.

Kuo, H. I., Bikson, M., Datta, A., Minhas, P., Paulus, W., Kuo, M. F., \& Nitsche, M. A. (2013). Comparing cortical plasticity induced by conventional and high-definition 4 x 1 ring tDCS: a neurophysiological study. Brain Stimul, 6(4), 644-648. doi:10.1016/j.brs.2012.09.010

Liebetanz, D., Koch, R., Mayenfels, S., Konig, F., Paulus, W., \& Nitsche, M. A. (2009). Safety limits of cathodal transcranial direct current stimulation in rats. Clin Neurophysiol, 120(6), 1161-1167. doi:10.1016/j.clinph.2009.01.022

Liebetanz, D., Nitsche, M. A., Tergau, F., \& Paulus, W. (2002). Pharmacological approach to the mechanisms of transcranial DC-stimulation-induced after-effects of human motor cortex excitability. Brain, 125(Pt 10), 2238-2247.

Lisman, J. E., \& Jensen, O. (2013). The theta-gamma neural code. Neuron, 77(6), 10021016. doi:10.1016/j.neuron.2013.03.007

Liu, S. K., Fitzgerald, P. B., Daigle, M., Chen, R., \& Daskalakis, Z. J. (2009). The relationship between cortical inhibition, antipsychotic treatment, and the symptoms 
$\begin{array}{llll}\text { of } & \text { schizophrenia. } & \text { Biol }\end{array}$ doi:10.1016/j.biopsych.2008.09.012

Loo, C. K., Sachdev, P., Martin, D., Pigot, M., Alonzo, A., Malhi, G. S., . . Mitchell, P. (2010). A double-blind, sham-controlled trial of transcranial direct current stimulation for the treatment of depression. Int J Neuropsychopharmacol, 13(1), 61-69. doi:10.1017/S1461145709990411

Manenti, R., Brambilla, M., Benussi, A., Rosini, S., Cobelli, C., Ferrari, C., . . Cotelli, M. (2016). Mild cognitive impairment in Parkinson's disease is improved by transcranial direct current stimulation combined with physical therapy. Mov Disord, 31(5), 715-724. doi:10.1002/mds.26561

McDonnell, M. N., Orekhov, Y., \& Ziemann, U. (2006). The role of GABA(B) receptors in intracortical inhibition in the human motor cortex. Exp Brain Res, 173(1), 86-93. doi:10.1007/s00221-006-0365-2

McQuail, J. A., Frazier, C. J., \& Bizon, J. L. (2015). Molecular aspects of age-related cognitive decline: the role of GABA signaling. Trends Mol Med, 21(7), 450-460. doi:10.1016/j.molmed.2015.05.002

Minzenberg, M. J., Laird, A. R., Thelen, S., Carter, C. S., \& Glahn, D. C. (2009). Metaanalysis of 41 functional neuroimaging studies of executive function in schizophrenia. Arch Gen Psychiatry, 66(8), 811-822. doi:10.1001/archgenpsychiatry.2009.91

Mondino, M., Jardri, R., Suaud-Chagny, M. F., Saoud, M., Poulet, E., \& Brunelin, J. (2016). Effects of Fronto-Temporal Transcranial Direct Current Stimulation on Auditory Verbal Hallucinations and Resting-State Functional Connectivity of the Left Temporo-Parietal Junction in Patients With Schizophrenia. Schizophr Bull, 42(2), 318-326. doi:10.1093/schbul/sbv114

Montgomery, S. A., \& Asberg, M. (1979). A new depression scale designed to be sensitive to change. Br J Psychiatry, 134, 382-389.

Mori, F., Ribolsi, M., Kusayanagi, H., Siracusano, A., Mantovani, V., Marasco, E., ... . Centonze, D. (2011). Genetic variants of the NMDA receptor influence cortical excitability and plasticity in humans. J Neurophysiol, 106(4), 1637-1643. doi:10.1152/jn.00318.2011

Nitsche, M. A., Doemkes, S., Karakose, T., Antal, A., Liebetanz, D., Lang, N., . . Paulus, W. (2007). Shaping the effects of transcranial direct current stimulation of the human motor cortex. J Neurophysiol, 97(4), 3109-3117. doi:10.1152/jn.01312.2006

Nitsche, M. A., Nitsche, M. S., Klein, C. C., Tergau, F., Rothwell, J. C., \& Paulus, W. (2003). Level of action of cathodal DC polarisation induced inhibition of the human motor cortex. Clin Neurophysiol, 114(4), 600-604.

Nitsche, M. A., \& Paulus, W. (2000). Excitability changes induced in the human motor cortex by weak transcranial direct current stimulation. J Physiol, 527 Pt 3, 633-639. 
Palm, U., Keeser, D., Schiller, C., Fintescu, Z., Nitsche, M., Reisinger, E., \& Padberg, F. (2008). Skin lesions after treatment with transcranial direct current stimulation (tDCS). Brain Stimul, 1(4), 386-387. doi:10.1016/j.brs.2008.04.003

Peinemann, A., Lehner, C., Conrad, B., \& Siebner, H. R. (2001). Age-related decrease in paired-pulse intracortical inhibition in the human primary motor cortex. Neurosci Lett, 313(1-2), 33-36.

Pelletier, S. J., \& Cicchetti, F. (2014). Cellular and molecular mechanisms of action of transcranial direct current stimulation: evidence from in vitro and in vivo models. Int J Neuropsychopharmacol, 18(2). doi:10.1093/ijnp/pyu047

Poreisz, C., Boros, K., Antal, A., \& Paulus, W. (2007). Safety aspects of transcranial direct current stimulation concerning healthy subjects and patients. Brain Res Bull, 72(46), 208-214. doi:10.1016/j.brainresbull.2007.01.004

Rabinowitz, J., Levine, S. Z., Garibaldi, G., Bugarski-Kirola, D., Berardo, C. G., \& Kapur, S. (2012). Negative symptoms have greater impact on functioning than positive symptoms in schizophrenia: analysis of CATIE data. Schizophr Res, 137(1-3), 147150. doi:10.1016/j.schres.2012.01.015

Radhu, N., de Jesus, D. R., Ravindran, L. N., Zanjani, A., Fitzgerald, P. B., \& Daskalakis, Z. J. (2013). A meta-analysis of cortical inhibition and excitability using transcranial magnetic stimulation in psychiatric disorders. Clin Neurophysiol, 124(7), 1309-1320. doi:10.1016/j.clinph.2013.01.014

Ranieri, F., Podda, M. V., Riccardi, E., Frisullo, G., Dileone, M., Profice, P., . . Grassi, C. (2012). Modulation of LTP at rat hippocampal CA3-CA1 synapses by direct current stimulation. J Neurophysiol, 107(7), 1868-1880. doi:10.1152/jn.00319.2011

Rawji, V., Ciocca, M., Zacharia, A., Soares, D., Truong, D., Bikson, M., . . Bestmann, S. (2018). tDCS changes in motor excitability are specific to orientation of current flow. Brain Stimul, 11(2), 289-298. doi:10.1016/j.brs.2017.11.001

Rogasch, N. C., \& Fitzgerald, P. B. (2013). Assessing cortical network properties using TMS-EEG. Hum Brain Mapp, 34(7), 1652-1669. doi:10.1002/hbm.22016

Rosler, K. M., Petrow, E., Mathis, J., Aranyi, Z., Hess, C. W., \& Magistris, M. R. (2002). Effect of discharge desynchronization on the size of motor evoked potentials: an analysis. Clin Neurophysiol, 113(11), 1680-1687.

Rossi, S., Hallett, M., Rossini, P. M., Pascual-Leone, A., \& Safety of, T. M. S. C. G. (2009). Safety, ethical considerations, and application guidelines for the use of transcranial magnetic stimulation in clinical practice and research. Clin Neurophysiol, 120(12), 2008-2039. doi:10.1016/j.clinph.2009.08.016

Rossini, P. M., Berardelli, A., Deuschl, G., Hallett, M., Maertens de Noordhout, A. M., Paulus, W., \& Pauri, F. (1999). Applications of magnetic cortical stimulation. The International Federation of Clinical Neurophysiology. Electroencephalogr Clin Neurophysiol Suppl, 52, 171-185. 
Sadock, B. J., Sadock, V. A., Ruiz, P., \& Kaplan, H. I. (2009). Kaplan \& Sadock's comprehensive textbook of psychiatry (Nona Edição ed.): Philadelphia : Wolters Kluwer Health/Lippincott Williams \& Wilkins.

Saha, S., Chant, D., Welham, J., \& McGrath, J. (2005). A systematic review of the prevalence of schizophrenia. PLoS Med, 2(5), e141. doi:10.1371/journal.pmed.0020141

Sarkar, S., Hillner, K., \& Velligan, D. I. (2015). Conceptualization and treatment of negative symptoms in schizophrenia. World $J$ Psychiatry, 5(4), 352-361. doi:10.5498/wjp.v5.i4.352

Schecklmann, M., Weidler, C., Eichhammer, P., Hajak, G., \& Langguth, B. (2018). Increased short-interval intracortical inhibition in un-medicated patients with schizophrenia. Brain Stimul. doi:10.1016/j.brs.2018.05.003

Schwenkreis, P., Witscher, K., Janssen, F., Addo, A., Dertwinkel, R., Zenz, M., . . . Tegenthoff, M. (1999). Influence of the N-methyl-D-aspartate antagonist memantine on human motor cortex excitability. Neurosci Lett, 270(3), 137-140.

Smith, R. C., Boules, S., Mattiuz, S., Youssef, M., Tobe, R. H., Sershen, H., . . Davis, J. M. (2015). Effects of transcranial direct current stimulation (tDCS) on cognition, symptoms, and smoking in schizophrenia: A randomized controlled study. Schizophr Res, 168(1-2), 260-266. doi:10.1016/j.schres.2015.06.011

Sommer, M., Ruge, D., Tergau, F., Beuche, W., Altenmuller, E., \& Paulus, W. (2002). Intracortical excitability in the hand motor representation in hand dystonia and blepharospasm. Mov Disord, 17(5), 1017-1025. doi:10.1002/mds.10205

Stagg, C. J., \& Nitsche, M. A. (2011). Physiological basis of transcranial direct current stimulation. Neuroscientist, 17(1), 37-53. doi:10.1177/1073858410386614

Strube, W., Bunse, T., Nitsche, M. A., Palm, U., Falkai, P., \& Hasan, A. (2016). Differential response to anodal tDCS and PAS is indicative of impaired focal LTPlike plasticity in schizophrenia. Behav Brain Res, 311, 46-53. doi:10.1016/j.bbr.2016.05.027

Tsapakis, E. M., Dimopoulou, T., \& Tarazi, F. I. (2015). Clinical management of negative symptoms of schizophrenia: An update. Pharmacol Ther, 153, 135-147. doi:10.1016/j.pharmthera.2015.06.008

Ucok, A., \& Ergul, C. (2014). Persistent negative symptoms after first episode schizophrenia: A 2-year follow-up study. Schizophr Res, 158(1-3), 241-246. doi:10.1016/j.schres.2014.07.021

Valiengo, L. C., Goulart, A. C., de Oliveira, J. F., Bensenor, I. M., Lotufo, P. A., \& Brunoni, A. R. (2017). Transcranial direct current stimulation for the treatment of post-stroke depression: results from a randomised, sham-controlled, double-blinded trial. J Neurol Neurosurg Psychiatry, 88(2), 170-175. doi:10.1136/jnnp-2016314075 
Valls-Sole, J., Pascual-Leone, A., Wassermann, E. M., \& Hallett, M. (1992). Human motor evoked responses to paired transcranial magnetic stimuli. Electroencephalogr Clin Neurophysiol, 85(6), 355-364.

van der Kamp, W., Zwinderman, A. H., Ferrari, M. D., \& van Dijk, J. G. (1996). Cortical excitability and response variability of transcranial magnetic stimulation. J Clin Neurophysiol, 13(2), 164-171.

van Haren, N. E., Cahn, W., Hulshoff Pol, H. E., \& Kahn, R. S. (2008). Schizophrenia as a progressive brain disease. Eur Psychiatry, 23(4), 245-254. doi:10.1016/j.eurpsy.2007.10.013

Wobrock, T., Schneider, M., Kadovic, D., Schneider-Axmann, T., Ecker, U. K., Retz, W., . . . Falkai, P. (2008). Reduced cortical inhibition in first-episode schizophrenia. Schizophr Res, 105(1-3), 252-261. doi:10.1016/j.schres.2008.06.001

Ziemann, U., Muellbacher, W., Hallett, M., \& Cohen, L. G. (2001). Modulation of practice-dependent plasticity in human motor cortex. Brain, 124(Pt 6), 1171-1181.

Ziemann, U., Paulus, W., Nitsche, M. A., Pascual-Leone, A., Byblow, W. D., Berardelli, A., . . . Rothwell, J. C. (2008). Consensus: Motor cortex plasticity protocols. Brain Stimul, 1(3), 164-182. doi:10.1016/j.brs.2008.06.006

Ziemann, U., Reis, J., Schwenkreis, P., Rosanova, M., Strafella, A., Badawy, R., \& MullerDahlhaus, F. (2015). TMS and drugs revisited 2014. Clin Neurophysiol, 126(10), 1847-1868. doi:10.1016/j.clinph.2014.08.028

Ziemann, U., Tam, A., Butefisch, C., \& Cohen, L. G. (2002). Dual modulating effects of amphetamine on neuronal excitability and stimulation-induced plasticity in human motor cortex. Clin Neurophysiol, 113(8), 1308-1315. 


\section{ANEXOS}

Anexo A. Parecer consubstanciado do comitê de ética em pesquisa, referente ao ensaio clínico STARTS.

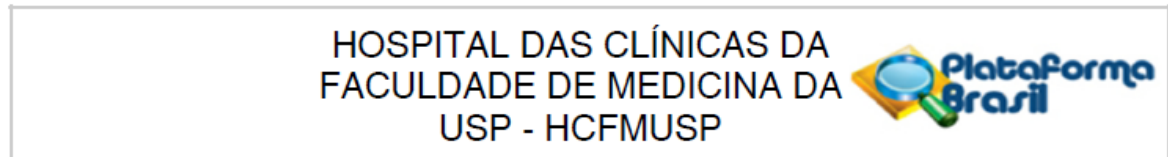

PARECER CONSUBSTANCIADO DO CEP

DADOS DO PROJETO DE PESQUISA

Título da Pesquisa: Tratamento de sintomas negativos da esquizofrenia com estimulação transcraniana por corrente contínua (ETCC): ensaio clínico, randomizado, sham-controlado, duplo-cego

Pesquisador: Wagner Farid Gattaz

Área Temática:

Versão:

CAAE: 31063714.1 .1001 .0068

Instituição Proponente: HOSPITAL DAS CLINICAS DA FACULDADE DE MEDICINA DA U S P

Patrocinador Principal: Financiamento Próprio

DADOS DO PARECER

Número do Parecer: 662.164

Data da Relatoria: $21 / 05 / 2014$

Apresentação do Projeto:

estudo randomizado, cego, controlado, multicêntrico, sobre tratamento de sintomas negativos de esquizofrenia com ETCC.

Objetivo da Pesquisa:

Resposta clínica de ETCC nos sintomas negativos na esquizofrenia

Avaliação dos Riscos e Benefícios:

Risco: mínimo: aplicação de questionários, coleta de sangue e aplicação de ETCC de baixa voltagem. Benefício: relevante- melhora clínica de doença cronica limitante incurável.

Comentários e Considerações sobre a Pesquisa:

$\mathrm{O}$ estudo é delineadao com rigor científico e apresentado de forma concisa e clara. O grupo tem expertize e recursos para desenvolver o projeto e publicar os resultados. O estudo é relevante.

Considerações sobre os Termos de apresentação obrigatória:

Foram apresentados: TCLE, projeto, folha de rosto adequadamente.

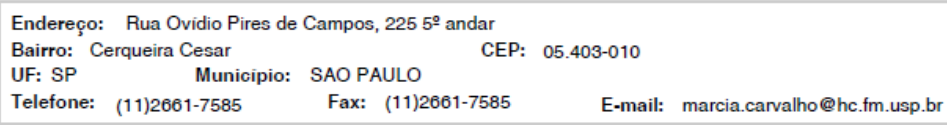




\section{HOSPITAL DAS CLÍNICAS DA FACULDADE DE MEDICINA DA USP - HCFMUSP}

Continuaçāo do Parecer: 662.164

\section{Recomendaçóes:}

Nenhuma

Conclusões ou Pendências e Lista de Inadequações:

Trabalho relevante e delineado com rigor científico por equipe com competência para sua execução.

Situação do Parecer:

Aprovado

Necessita Apreciação da CONEP:

Não

Considerações Finais a critério do CEP:

Em conformidade com a Resolução CNS n 466/12 - cabe ao pesquisador: a) desenvolver o projeto conforme delineado; b) elaborar e apresentar relatórios parciais e final; c)apresentar dados solicitados pelo CEP, a qualquer momento; d) manter em arquivo sob sua guarda, por 5 anos da pesquisa, contendo fichas individuais e todos os demais documentos recomendados pelo CEP; e) encaminhar os resultados para publicação, com os devidos créditos aos pesquisadores associados e ao pessoal técnico participante do projeto; f) justificar perante ao CEP interrupção do projeto ou a não publicação dos resultados.

SAO PAULO, 27 de Maio de 2014

Assinado por:

ALFREDO JOSE MANSUR

(Coordenador)

Endereço: Rua Ovidio Pires de Campos, $2255^{\circ}$ andar

$\begin{array}{lll}\text { Bairro: } & \text { Cerqueira Cesar } & \\ \text { UF: SP } & \text { Municipio: SAO PAULO }\end{array}$

Telefone: (11)2661-7585

Fax: (11)2661-7585

E-mail: marcia.carvalho@hc.fm.usp.br 
Anexo B. Atestado de matrícula e conclusão de 2 semestres de doutoramento na Universidade Eberhard Karl, Tübingen, Alemanha (na qualidade de doutorando visitante)

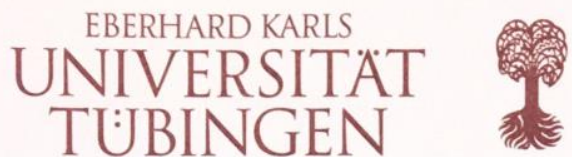
Zentrale Verwaltung
II - Studium und Lehre
Studentensekretariat

\author{
Universitat Tubingen, ZV II 1 Wilhelmstr. 11, 72074 Tubingen \\ Herrn \\ Pedro Caldana Gordon \\ Herrenberger Str. 11 \\ D 72070 Tübingen
}

\title{
Exmatrikulationsbescheinigung
}

Herr Pedro Caldana Gordon,

geboren am 25.04.1983 in Sao Paulo, war vom 07.11.2016 bis zum 30.09.2017 unter der Matrikelnummer 4118286 an der Eberhard Karls Universität Tübingen immatrikuliert.

Grund seiner Exmatrikulation: sonstige Gründe.

Dieser Grund entspricht der Angabe zum Zeitpunkt der Exmatrikulation.

Herr Caldana Gordon befand sich zum Zeitpunkt der Exmatrikulation

im 2. Hochschulsemester und war 0 Semester beurlaubt.

Zuletzt belegte Studienfächer:

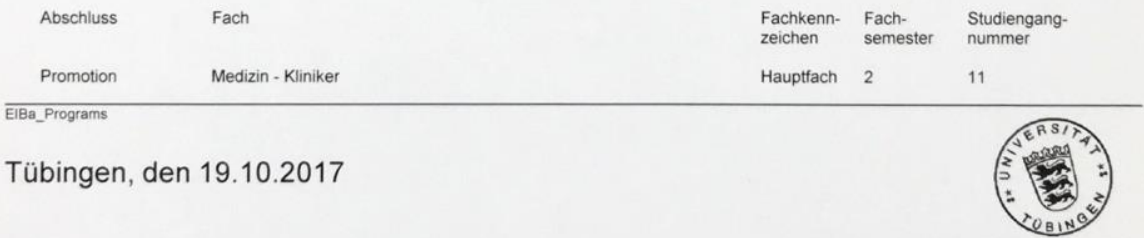

Alle Prüfungsleistungen sind durch entsprechende Zeugnisse der jeweiligen Prüfungsämter nachzuweisen Zusätze und Änderungen müssen vom Studentensekretariat mit Unterschrift und Dienstsiegel bestätigt werden. 
Anexo C. Produção científica

Gordon PC, Zrenner C, Desideri D, Belardinelli P, Zrenner B, Brunoni AR, Ziemann U. Modulation of cortical responses by transcranial direct current stimulation of dorsolateral prefrontal cortex: A resting-state EEG and TMS-EEG study. Brain Stimul. 2018 Sep Oct;11(5):1024-1032. doi: 10.1016/j.brs.2018.06.004. Epub 2018 Jun 18. 


\section{Modulation of cortical responses by transcranial direct current stimulation of dorsolateral prefrontal cortex: A resting-state EEG and TMS-EEG study}

Pedro Caldana Gordon ${ }^{\mathrm{a}, \mathrm{b}}$, Christoph Zrenner ${ }^{\mathrm{a}}$, Debora Desideri ${ }^{\mathrm{a}}$, Paolo Belardinelli ${ }^{\mathrm{a}}$, Brigitte Zrenner ${ }^{\mathrm{a}}$, André Russowsky Brunoni b, c, Ulf Ziemann ${ }^{\mathrm{a}, *}$

a Department of Neurology \& Stroke, Hertie Institute for Clinical Brain Research, University of Tübingen, Hoppe-Seyler-Straße 3, 72076, Tübingen, Germany bervice of Interdisciplinary Neuromodulation, Laboratory of Neuroscience (UM27) and National Institute of Biomarkers in Psychiatry (INBioN), Department and Institute of Psychiatry, Hospital das Clinicas HCFMUSP, Faculdade de Medicina, Universidade de São Paulo, R Dr. Ovídio Pires de Campos 785, 01060-970, São Paulo, Brazil

'Department of Psychiatry and Psychotherapy, Ludwig-Maximilians-University, Leopoldstr. 13, 80802, Munich, Germany

A R T I C L E I N F O

Article history:

Received 2 March 2018

Received in revised form

16 May 2018

Accepted 7 June 2018

Available online $x x x$

\section{Keywords:}

Transcranial direct current stimulation

Neuromodulation

Transcranial magnetic stimulation

Electroencephalogram

Dorsolateral prefrontal cortex

\begin{abstract}
A B S T R A C T
Background: Transcranial direct current stimulation (tDCS) is a non-invasive brain stimulation technique with potential for cost-effective therapeutic neuromodulation. Although positive therapeutic effects were found by stimulating the dorsolateral prefrontal cortex (DLPFC), few studies have investigated physiological effects of DLPFC-tDCS.

Objectives: To investigate effects of tDCS with different parameter settings applied to the left DLPFC on cortical responses, measured by resting-state electroencephalography (rs-EEG) and transcranial magnetic stimulation (TMS)-evoked/induced EEG responses.

Methods: 22 healthy subjects underwent 5 tDCS sessions with different tDCS parameter settings in a double-blinded randomized crossover design (1: $1.5 \mathrm{~mA}$, anode left-DLPFC, cathode right-DLPFC; 2 : $1.5 \mathrm{~mA}$, cathode left-DLPFC, anode right-DLPFC; 3: $0.5 \mathrm{~mA}$, anode left-DLPFC, cathode right-DLPFC; 4 : $1.5 \mathrm{~mA}$, anode left-DLPFC, cathode left deltoid muscle; 5: sham stimulation). Rs-EEG and TMS-EEG were recorded before and after tDCS.

Results: Rs-EEG power spectrum analysis showed no difference comparing baseline with post stimulation in any of the tDCS conditions. TMS-EEG evoked potential amplitude decreased in parietal cortex after $1.5 \mathrm{~mA}$ left-DLPFC anodal tDCS, and TMS-induced gamma and theta oscillations decreased after al conditions using left-DLPFC anodal tDCS. Left-DLPFC cathodal tDCS did not lead to significant change. None of the post-intervention changes was different when comparing the effects across conditions, including sham.

Conclusions: Our study does not provide evidence that a single tDCS session results in significant changes in rs-EEG, using the current stimulation parameters. Significant changes in EEG responses to TMS pulses were observed following the anodal $1.5 \mathrm{~mA}$ tDCS interventions, although these changes were not statistically significant in a group comparison.
\end{abstract}

C 2018 Elsevier Inc. All rights reserved.

\section{Introduction}

Transcranial direct current stimulation (tDCS) is a non-invasive brain stimulation technique that has gained much attention due to its potential clinical applications [1], coupled with low cost and

\section{* Corresponding author.}

E-mail address: ulf.ziemann@uni-tuebingen,de (U. Ziemann). favorable safety profile $[2,3]$. The prospect of a highly cost-effective neuromodulation tool has led to the investigation of numerous possible usages of this technique. Nevertheless, only a few clinical conditions, such as major depressive disorder, have been shown to consistently improve following tDCS treatment $[4,5]$. The neuromodulatory potential of tDCS has also been investigated in cognitive tasks, with reports of increased working memory performance after tDCS [6], although only few experiments consistently showed a lasting effect of tDCS on cognition [7]. The lack of consistency of

https://doi.org/10.1016/j.brs,2018.06.004

1935-861X/0 2018 Elsevier Inc. All rights reserved.

Please cite this article in press as: Gordon PC, et al., Modulation of cortical responses by transcranial direct current stimulation of dorsolateral prefrontal cortex: A resting-state EEG and TMS-EEG study, Brain Stimulation (2018), https://doi.org/10.1016/j.brs.2018.06.004 
reported effects derived from tDCS trials has been partially attributed to high variability of stimulation parameters across studies, as well as other factors, such as age, gender, and brain state during neuromodulation $[7,8]$.

The basic operation of tDCS involves the induction of a lowintensity electrical field in the brain by injecting electrical current, usually between 1 and $2 \mathrm{~mA}$, through a set of electrodes placed on the subject's scalp [9]. It has been hypothesized that the electric field induced by tDCS shifts the polarity difference between the intra- and extracellular space, which either increases neuronal firing rate (anodal stimulation) or decreases it (cathodal stimulation) [10,11]. In human studies, tDCS applied over motor cortex was shown to modulate motor evoked potentials (MEP) elicited by transcranial magnetic stimulation (TMS), with anodal stimulation increasing MEP amplitude and cathodal stimulation decreasing it [12,13]. Although changes in MEP amplitude after a single session tend to be short lasting, longer duration of tDCS led to longer lasting after-effects [14], supporting the idea that repetitive sessions of tDCS would lead to more stable and enduring neuroplasticity $[15,16]$. However, the effects of tDCS on cortical modulation were found to be less predictable than expected, as changes of stimulation parameters would promote significant shifts in expected cortical responses. In the motor cortex, a 26-min session (vs. 13 min) led to effects opposite from the expected, with anodal tDCS of motor cortex decreasing MEP amplitude [16]. Cathodal tDCS over motor cortex, initially believed to induce MEP amplitude reduction [12], led to MEP increase when a higher current intensity ( $2 \mathrm{~mA}$ rather than $1 \mathrm{~mA}$ ) was applied [17]. Brain state was also observed to impact the effects of tDCS, as the same stimulation parameters led to different results, depending on whether subjects were at rest or performing a cognitive task during stimulation [18].

Furthermore, most studies investigating tDCS effects on the human brain targeted the motor cortex. This is a relevant limitation for the application of tDCS in clinical practice, as most cortical targets in neuropsychiatry and cognition are non-motor areas [5,7]. Also, given the high variability of cortical reactivity in response to tDCS protocols on motor cortex, there is little reason to expect that tDCS of non-motor cortex will follow a simple "anode-facilitation cathode-inhibition" model. Moreover, an important limitation of neurophysiological studies in non-motor cortical areas is the absence of a direct read-out of cortical excitability, easily accessed in motor cortex through MEP amplitude. An alternative way to quantify cortical modulation in non-motor areas is by means of measuring scalp potentials with electroencephalography (EEG). RsEEG signal analysis has been used to investigate effects of tDCS applied to the frontal cortex. The available studies have described diverse changes in the EEG signal following tDCS to the prefrontal cortex, such as increase in medial prefrontal theta power [19], or change in the mean frequency index [20], while other studies found no change of rs-EEG following $\operatorname{tDCS}[21,22]$, indicating an overal lack of evidence for an effect of tDCS on rs-EEG [23]. TDCS-related changes in cortical excitability were also probed using TMS-EEG (transcranial magnetic stimulation coupled with EEG), a complementary technique that allows direct analysis of TMS-evoked induced cortical responses to stimulation of any cortical area [24]. Several authors [25-27] investigated the effects of tDCS over the motor cortex with TMS-EEG, and reported a significant amplitude increase of TMS evoked EEG potentials (TEPS) after anodal tDCS, and a decrease after cathodal tDCS, both concomitant with the expected changes in MEP amplitude. A further study of tDCS over the parietal cortex also showed modulation of cortical excitability measured with TMS-EEG, with increased TEP amplitudes after anodal tDCS [28]. Finally, a study using bipolar vs. high-definition tDCS (HD-tDCS), targeting the dorsolateral prefrontal cortex
(DLPFC), found significant modulation of cortical excitability measured with TMS-EEG [29].

Despite these initial findings, it is not yet clear how the DLPFC reacts to different tDCS settings. A careful appraisal of the difference in cortical responses to varying tDCS parameters, analogous to motor cortex $[16,17]$, would provide relevant information of the DLPFC responsivity to tDCS. To this aim, we have tested the effects of 4 different set of tDCS parameters over the DLPFC, and investigated the changes in cortical activity and response using rs-EEG and TMS-EEG. Considering previous findings regarding cortical excitability of the motor cortex, our aims were to address in the DLPFC: (1) polarity-dependent effects by testing anodal (associated with increased cortical excitability) vs. cathodal tDCS (associated with decreased cortical excitability); (2) current-strength-dependent effects, by testing $0.5 \mathrm{~mA}$ (associated with decreased cortica excitability) vs. $1.5 \mathrm{~mA}$ anodal tDCS (associated with increased cortical excitability) and; (3) the influence of the placement of the return electrode, by testing a cephalic vs. extra-cephalic montage.

\section{Materials and methods}

\subsection{Subjects}

22 healthy right-handed volunteers (12 females, mean age: $26.9 \pm 8.2$ years) completed all sessions and their data were included in the present study. Right-handedness was confirmed using the Edinburgh Handedness Inventory (laterality score $\geq 75 \%$ ) [30]. Exclusion criteria were prior history of psychiatric or neurological disease, current treatment with drugs acting on central nervous system, presence or prior history of alcohol or illicit drugs abuse, and current pregnancy. The study was approved by the local Ethics Committee of the Medical Faculty of the Eberhard-KarlsUniversity Tübingen, and all subjects provided written informed consent prior to participation.

\subsection{Design}

The study followed a randomized, sham-controlled, doubleblinded, repeated measures design. Each subject underwent 5 sessions, with a one-week lapse between sessions. Each session consisted of baseline measurements (BASELINE) followed by tDCS intervention, and immediately followed by outcome measurements (POST). Measurements consisted of resting state EEG (rsEEG) and TMS evoked responses, as described below. For a given subject, all sessions were conducted on the same week day and time of day.

\subsection{Transcranial direct current stimulation}

tDCS was applied using a DC stimulator (DC-Stimulator Plus, neuroCare Group GmbH, Germany). The five sessions involved the following parameter settings: \#1: $1.5 \mathrm{~mA}$, anode left-DLPFC, cathode right-DLPFC; \#2: $1.5 \mathrm{~mA}$, cathode left-DLPFC, anode rightDLPFC; \#3: $0.5 \mathrm{~mA}$, anode left-DLPFC, cathode right-DLPFC; \#4: $1.5 \mathrm{~mA}$, anode left-DLPFC, cathode left deltoid muscle; \#5: sham stimulation (Fig. 1B). The order in which these sessions were delivered to each subject was randomized. All tDCS interventions lasted 14 min each, including $30 \mathrm{~s}$ of electric current ramp-up at the beginning of the stimulation and $10 \mathrm{~s}$ ramp-down at the end. In the sham condition the current was reduced to zero after the 30 -s ramp-up. This procedure was shown to effectively simulate an active tDCS session, thus assuring that subjects remained blinded to the stimulation condition [31]. 
A.

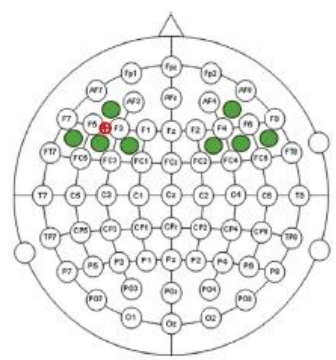

C.
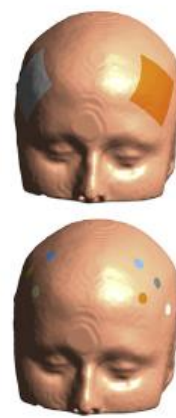

B.

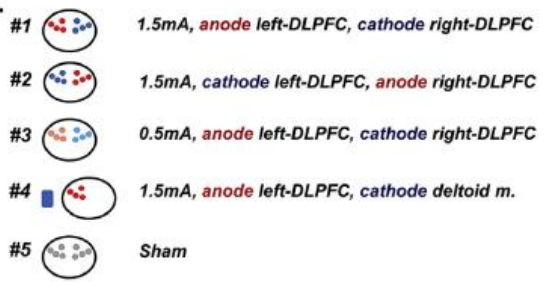

45

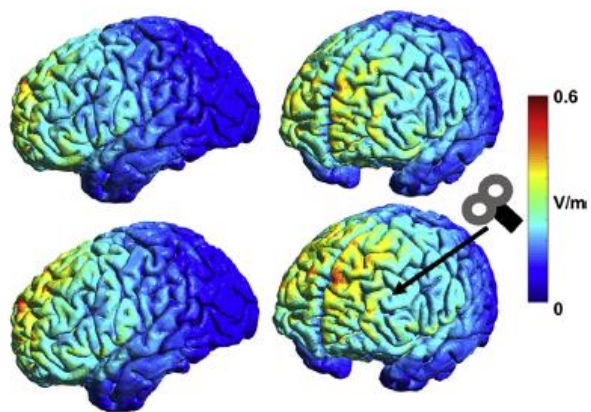

Fig. 1. A. Diagram of the 64 EEG electrodes cap used in the experiments; green circles represent the position of the tDCS electrodes, the red circle represents the estimated location of the left DLPFC and the target for single-pulse TMS. B. Diagrams of the 5 tDCS parameter settings used in the present experiments; red circles represent the anode, blue circles represent the cathode, orange (anode) and light blue circles (cathode) in \#3 indicate low stimul ation intensity ( $0.5 \mathrm{~mA}$ ), blue rectangle in \# 4 represents the extra-cephalic cathode over left deltoid muscle, and grey circles in \#5 the sham stimulation. C. Computational modeling (using SimNIBS) of the electric field strength generated by tDCS montages over

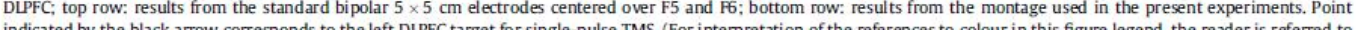
indicated by the black arrow corresponds to the left DLPFC target for single-pulse TMS. (For interpretation of the references to colour in this figure legend, the reader is referred to the Web version of this article.)

Most of the clinical evidence for tDCS efficacy in neuropsychiatric conditions is derived from trials using standard bipolar montage, involving rectangular electrodes [5,32]. Our aim was to investigate the effects of tDCS sessions with same settings used in trials, which would therefore require the use of $25 \mathrm{~cm}^{2}$ or $35 \mathrm{~cm}^{2}$ rectangular electrodes on the scalp. However, the use of such electrodes would hinder EEG recordings from the stimulated site, due to creation of current bridges between EEG electrodes and consequently invalidating the recording from the very region under investigation. To overcome this drawback, we designed a montage using clustered small tDCS electrodes, placed between EEG channels, thus simulating the standard bipolar tDCS montage $[4,33]$ This set-up was implemented by means of eight round tDCS electrodes (diameter: $1 \mathrm{~cm}$ ), integrated between the EEG sensors of a standard 64 channel EEG cap, centered around the estimated location of the DLPFC using the Standard International EEG system, i.e. the EEG channels F3 and F5 on left hemisphere and F4 and F6 on right hemisphere [34]. The application of small tDCS electrodes in between EEG electrodes for similar purposes has been successfully demonstrated $[35,36]$. Schematics of the tDCS electrodes locations on the scalp are shown in Fig. 1A.

\subsection{Computational modeling of electric fields}

We performed a simulation of electric field strength induced by tDCS, using the SimNIBS modeling environment, Simulation of Non-Invasive Brain Stimulation [37], and the sample brain model included in the software. The aim of this procedure was to test to what extent the distribution of the electric field over the prefrontal cortex yielded by the electrode montage used in the present experiment is comparable to the standard bipolar montage with large patch electrodes. The following describes the set of parameters used in the computational model: The standard montage was designed by modeling $5 \times 5 \mathrm{~cm}$ electrodes of $1.0 \mathrm{~mm}$ thickness and $2.0 \mathrm{~mm}$ sponge, centered at the coordinates corresponding to the F5 and F6 electrode of the International 10-20 EEG system, and $1 \mathrm{~mA}$ current intensity. The montage used in the present experiments was modelled with a set of 8 electrodes, $1 \mathrm{~cm}$ diameter with $1.0 \mathrm{~mm}$ thickness and $2.0 \mathrm{~mm}$ gel layer each. The coordinates for placement of the simulated electrodes followed the 128 channels Intemational EEG system with AFF5h, FFT7h, FFC5h, FFC3h for the anode, and AFF6h, FFT8h, FFC6h, FFC4h for the cathode (Fig. 1A). Current intensity was modelled at $0.25 \mathrm{~mA}$ for each electrode, summing up to a total of $1.0 \mathrm{~mA}$ running from the cathode to the anode electrode cluster.

\subsection{Transcranial magnetic stimulation}

TMS was delivered using a Magstim Super Rapid ${ }^{2}$ Plus $^{1}$ Stimulator (Magstim Co. Ltd., UK) connected to a figure-of-eight coil with internal wing diameter of $70 \mathrm{~mm}$ (D70 mm Remote Coil, Magstim Co. Ltd., UK), using biphasic pulses in AP-PA configuration. Resting motor threshold (RMT) was assessed at the beginning of the experiments, with surface EMG over the right abductor pollicis brevis 
muscle and positioning the coil over the left primary motor cortex (M1) hand area, determined as the lowest intensity with which at least 5 out of 10 MEPs exceeded a peak-to-peak amplitude of $50 \mu \mathrm{V}$ [38]. The target for the TMS evoked responses was the left DLPFC, defined in the present experiment according to the coordinates ( $\mathrm{x}$ $y, z)=-50,30,36$ (MNI brain) [34]. For each session, the subject's head surface was fitted into a preexisting MNI model using a neuronavigation system (Localite, Localite GmbH, Germany), and the left DLPFC coordinates were set as the TMS target. Neuronavigation trackers were placed both on the subject's head and on the TMS coil for continuous online monitoring to ascertain correct coil positioning throughout the experiment with respect to the designated target. This procedure was also relevant for consistency of the stimulation site across BASELINE and POST measurements, as well as across the 5 sessions. During the recordings, subjects were seated on a comfortable reclining chair, and asked to look at a fixation cross. Both BASELINE and POST measurements consisted of 5 min eyes opened rs-EEG recording, followed by the application of 160 single TMS pulses over the target area, at an average rate of $0.2 \mathrm{~Hz}$ (randomly jittered intertrial interval of $5 \pm 2 \mathrm{~s}$ ), and a stimulation intensity of $120 \%$ of RMT.

\subsection{Resting-state EEG and TMS-EEG recordings}

TMS-evoked EEG potentials (TEPs) were recorded using a TMScompatible EEG system (Bittium NeurOne, Bittium Corporation, Finland), which prevents EEG amplifier from saturation and allows continuous data recording during TMS. EEG signal was digitized at a sampling frequency of $5 \mathrm{kHz}$ and continuously recorded by 64 electrodes mounted on an EEG cap according to the standard 10-20 layout. Impedance of all electrodes was kept below $5 \mathrm{k} \Omega$ throughout the experiment.

\subsection{Data analysis}

Data analysis was performed on the MATLAB platform (R2017b The Mathworks, USA), using Fieldtrip open source toolbox [39] and customized Matlab scripts. Data from the BASELINE and POST measurements of a given session were processed together. Rs-EEG data was down-sampled to $1 \mathrm{kHz}$ and segmented into $3 \mathrm{~s}$ epochs. Single epochs were visually examined, epochs and channels with excessive noise were excluded. Independent component analysis (ICA) based on FastICA algorithm [40] was performed to exclude components containing blink, eye movement and muscle artifacts. Channels excluded due to excessive noise were then splineinterpolated [41]. Data was then referenced to the average of all electrodes. Power density was estimated by means of the fast Fourier transform (multitaper method) for frequencies ranging from 4 to $48 \mathrm{~Hz}$, and further log-transformed. The log-transformed power of the signal from each trial was then divided into the following frequency bands: $4-7 \mathrm{~Hz}$ (theta), $8-12 \mathrm{~Hz}$ (alpha), $13-29 \mathrm{~Hz}$ (beta) and $30-48 \mathrm{~Hz}$ (gamma). The power values of all epochs for each measurement were then averaged. For the region of interest (ROI) analysis, power values from channels F3 and F5 were averaged.

For the TMS evoked responses analysis, EEG data were segmented into epochs centered on the TMS pulse $(-1000$ to $1500 \mathrm{~m} \mathrm{~s}$ ) and then baseline corrected ( -500 to $-100 \mathrm{~m} \mathrm{~s})$. Data containing the TMS pulse and associated muscle activity $(-2$ to $+25 \mathrm{~m} \mathrm{~s}$ window around each TMS pulse) was removed and cubic interpolated. As with rs-EEG data, epochs were visually examined, and epochs and channels with excessive noise were excluded. Further artifacts were removed with a 2-step based ICA procedure $[42,43]$. A first round of ICA was performed to remove components containing a large amplitude exponential decay, associated with the TMS elicited muscle response. A band pass filter $(1-100 \mathrm{~Hz})$ and a band stop filter $(49-51 \mathrm{~Hz})$ were applied, followed by a second round of ICA, to remove components containing blink, eye movement and muscle artifacts. Data was then referenced to the average of all electrodes.

For the TEP analysis, the EEG data was lowpass filtered $(45 \mathrm{~Hz}$, zero-phase Butterworth, 3rd order) and averaged across epochs for further statistical testing. For the TMS induced oscillations analysis, time-frequency representations (TFRs) of TMS-related oscillatory power changes were calculated using a Morlet wavelet decomposition on single trials [44], with frequencydependent width (wavelet width of 2.6 cycles at $4 \mathrm{~Hz}$, adding 0.2 cycle for each $1 \mathrm{~Hz}$ ). The time-frequency oscillatory response to TMS can be divided into an evoked response (i.e. phase-locked to TMS) and an induced response (i.e. non-phase-locked TMS) $[45,46]$. The TFR of the induced response was isolated by subtracting the individual time-domain average from each trial before calculating the TFRs of the single trials [46]. This was followed by the individual trial normalization for each frequency, based on a z-transformation that used the trial's respective mean and standard deviation for the power of each frequency from the full trial length. This procedure transforms all power data to the same scale, allowing comparison across participants, trials and electrodes [29]. An absolute baseline correction for each trial was then applied by subtracting the average of the -500 to $-100 \mathrm{~m} \mathrm{~s}$ period (baseline) for each frequency. A period no closer than $-100 \mathrm{~ms}$ to the TMS was chosen to avoid possible contamination from the TMS artifact. This procedure aimed to ensure that the $z$-values represent a change in cortical oscillations following the TMS pulse, compared to the pre-TMS baseline $[46,47]$. Finally, TFRs were averaged across trials, followed by statistical testing as indicated below.

\subsection{Statistical analyses}

All statistical analyses were performed on the MATLAB platform (R2017b, The Mathworks, USA). Rs-EEG data was analyzed by focusing on a ROI, set as the average signal from the electrodes F3 and F5. We also applied cluster based statistics, a nonparametric permutation test based on the Monte-Carlo method, aimed to correct for multiple comparisons due to the large number of electrodes and time and/or frequency points [48]. To test whether the BASELINE measurements across interventions were comparable, a one-way analysis of variance (ANOVA) was applied, having as dependent measures the spectral power in each frequency band at the ROI. Following this, a repeatedmeasures analysis of variance (rmANOVA) was run, including 2 factors: TIME (two levels, BASELINE and POST) and CONDITION (5 levels, the different tDCS interventions), with the dependent variable the measure of spectral power in each frequency band for rs-EEG. Cluster based t-tests were used to compare the difference in spectral power in each frequency between BASELINE and POST in each condition.

TEPs and Induced Oscillations were first analyzed using a cluster based one-way ANOVA to test whether the BASEUINE measurements across interventions were comparable. Differences between BASELINE and POST measurements in each condition was tested using cluster based $t$-test, comparing TEP amplitude for the TEP analysis, covering a latency of $30-300 \mathrm{~m} \mathrm{~s}$ after TMS, and spectral power for induced oscillations analysis, covering a latency of $30-400 \mathrm{~ms}$ after TMS, and divided into 4 different frequency bands: $4-7 \mathrm{~Hz}$ (theta), $8-12 \mathrm{~Hz}$ (alpha), $13-29 \mathrm{~Hz}$ (beta) and $30-48 \mathrm{~Hz}$ (gamma). Finally, a comparison across conditions was performed by a one-way ANOVA, having as independent effects the $5 \mathrm{tDCS}$ conditions and as dependent measure the difference post- 
Table 1

RmANOVA with dependent measure of rs-EEG power spectrum in each frequency band at the ROL, and time (BASELINE vc. POST) and intervention (5 tDCS sessions) as independent main effects.

\begin{tabular}{|c|c|c|c|c|c|}
\hline Frequency Band & & Sum of Squares & (df intercept, df error) & $\mathbf{F}$ & $\mathrm{p}$ \\
\hline \multirow[t]{3}{*}{ THETA } & Time & 12.41 & $(1,21)$ & 0.22 & 0.642 \\
\hline & Intervention & 461.23 & $(4,84)$ & 1.38 & 0.248 \\
\hline & Time"Intervention & 109.14 & $(4,84)$ & 2.12 & 0.084 \\
\hline \multirow[t]{3}{*}{ ALPHA } & Time & 138.11 & $(1,21)$ & 1.73 & 0.201 \\
\hline & Intervention & 81.06 & $(4,84)$ & 0.12 & 0.973 \\
\hline & Time Intervention & 95.17 & $(4,84)$ & 1.16 & 0.330 \\
\hline \multirow[t]{3}{*}{ BETA } & Time & 991.95 & $(1,21)$ & 2.33 & 0.141 \\
\hline & Intervention & 7340.8 & $(4,84)$ & 1.16 & 0.333 \\
\hline & Time'Intervention & 579.85 & $(4,84)$ & 0.75 & 0.555 \\
\hline \multirow{3}{*}{ GAMMA } & Time & 96.03 & $\begin{array}{l}(1,21) \\
(7,21)\end{array}$ & 0.43 & 0.518 \\
\hline & Intervention & 11462 & $(4,84)$ & 1.59 & 0.182 \\
\hline & Time Intervention & 1887.9 & $(4,84)$ & 1.77 & 0.142 \\
\hline
\end{tabular}

vs. pre-intervention: $\triangle$ TEPs (POST amplitude minus BASELINE amplitude), and $\Delta$ Induced Oscillations (POST spectral power minus BASELINE spectral power). For cluster based statistics, a minimum number of 2 channels was established as a threshold for cluste size, and the number of permutations for the Monte Carlo method was 1000 for rs-EEG and TEPs, and 2000 for Induced Oscillations.

\section{Results}

3.1. Computational modeling of electric fields

Computational modeling showed a diffusely distributed electric field over the frontal cortices, encompassing the left DLPFC, with the peak field extending from the targeted area towards the midline, in both the standard montage and the montage used in the present study (Fig. 1C). Results obtained from the standard montage over the prefrontal cortex are similar to previous reports [33,49]. Nonetheless the electric field distribution from the presen study's montage is not identical to the standard montage, as sparse areas of higher amplitudes were observed, likely due to higher current densities produced by the small electrode's area $(1 \mathrm{~mA}$ standard montage: $0.04 \mathrm{~A} / \mathrm{cm}^{2}$; present study montage: $0.31 \mathrm{~A}$ $\left.\mathrm{cm}^{2}\right)$.

\subsection{Resting-state EEG}

No significant difference of power was found comparing the BASELINE measurements across the different tDCS conditions, in any frequency band of interest. Moreover, none of the tDCS interventions resulted in a significant change of spectral power POST vs. BASELINE in any frequency band (Table 1). Fig. 2 shows the average rs-EEG power spectrum of all 22 subjects in the ROI (electrodes F3, F5) at baseline and post-tDCS, separately for the five tDCS interventions. Cluster based analysis also failed to show any region or frequency band with significant power difference POST vs. BASELINE. A further statistical approach using a linear mixed effect model was attempted, again without significant findings (Supplementary Material, Tables S1-S4).

\subsection{TMS-evoked EEG potentials (TEPS)}

Single-pulse TMS over the left DLPFC resulted in a series of deflections of the EEG signal, which have been classified in previous studies according to their latency [26,42]. The same pattern can be observed in the present data (Fig. 3).

No significant differences between tDCS conditions were found at BASELINE. Pairwise comparisons with cluster-based t-tests revealed that only condition \#1 (1.5 mA, anode left-DLPFC, cathode

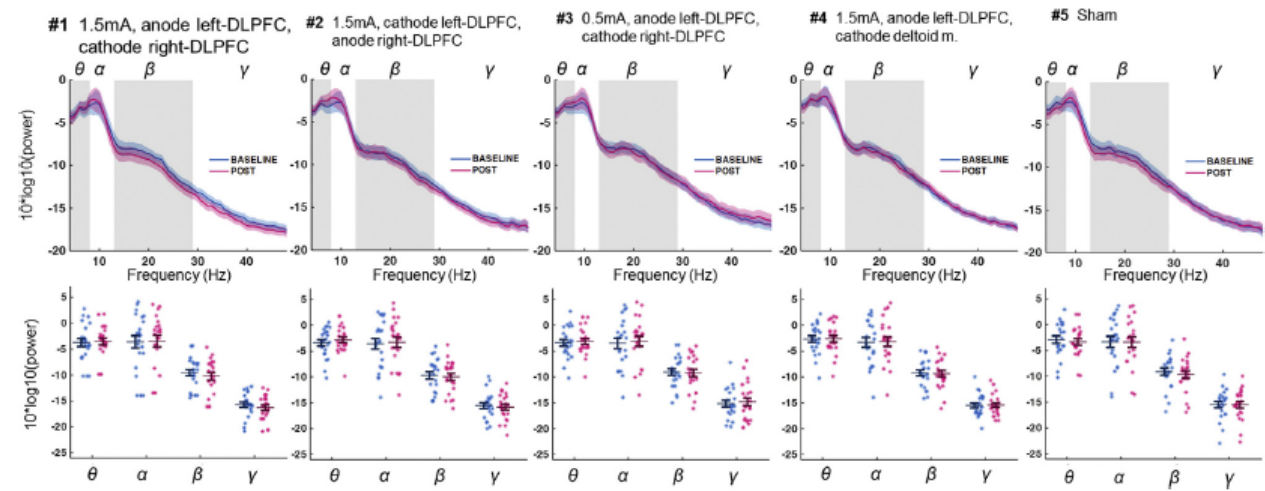

Fig. 2. Top: Average ( $\mathrm{n}=22$ subjects) of the rs-EEG power spectrum in the ROI (electrodes F3, F5), BASELINE (blue) and POST (red). Each plot represents one of the five tDCS conditions (see plot headers) used in this study. Frequency bands are highlighted ( $\theta$ :theta, $\alpha$ :alpha, $\beta$ :beta, $\gamma$ :gamma). Curve shadings are \pm 1 SEM. Bottom: Individual data of average power of each frequency band, BASELINE (blue) and POST (red). Means across subjects are indicated as long horizontal bars, short horizontal lines correspond to \pm 1 SEM. (For interpretation of the references to colour in this figure legend, the reader is referred to the Web version of this article.)

Please cite this article in press as: Gordon PC, et al., Modulation of cortical responses by transcranial direct current stimulation of dorsolateral prefrontal cortex: A resting-state EEG and TMS-EEG study, Brain Stimulation (2018), https://doi.org/10.1016/j.brs.2018.06.004 
A.

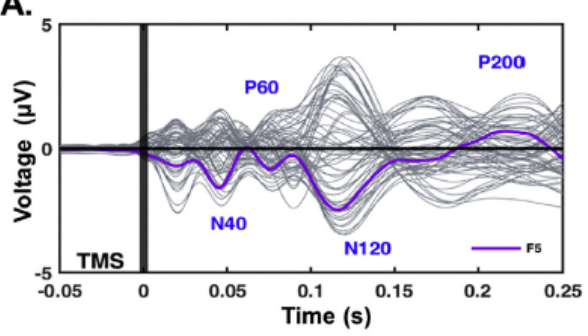

B.

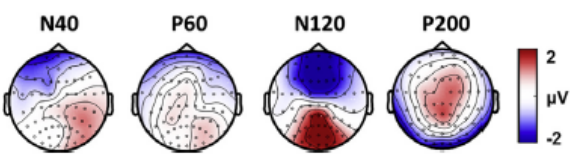

Fig. 3. A. Butterfly plot of the grand average across all subjects $(n=22)$ and five tDCS interventions at BASEUNE of the TMS evoked EEF potentials. The purple curve is the signal recorded from electrode F5. TEP peaks are indicated according to their polarities and latencies (N40, P60, N120 and P200). B. Spatial distribution of voltage over the scalp corresponding to each of the major TEP peaks. (For interpretation of the references to colour in this figure legend, the reader is referred to the Web version of this article.)

right-DLPFC) resulted in a significant difference, with a TEP amplitude reduction in the $90-200 \mathrm{~m}$ s period after the TMS pulse, involving a cluster centered in the parietal midline region, as shown in Fig. 4 and Table 2. However, there was no significant difference when comparing the TEP amplitude differences ( $\triangle \mathrm{TEPS}$ ) between POST and BASELINE across conditions within that period. No other significant $\triangle T E P s$ clusters were found at any other time after the TMS pulse or tDCS condition (Table 3).

$\# 1$

$1.5 \mathrm{~mA}$,

Anode left-DLPFC

Cathode left-DLPFC

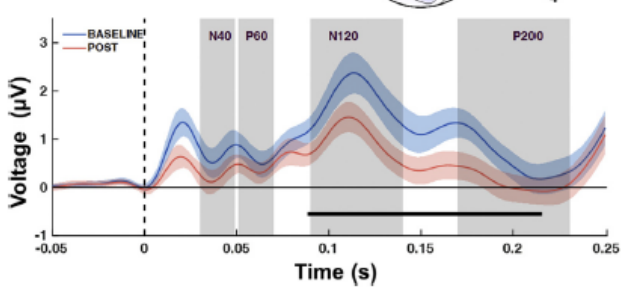

Fig. 4. On top, topographical plot of the statistical difference ( $\mathrm{t}$-values) between POST and BASEUNE voltages in the significant cluster, tDCS intervention \#1. Electrodes highlighted by red crosses belong to the cluster, in which the statistical significance was found $(* p=0.006)$. At the bottom, time plot of the average voltage from this EEC channel cluster, background areas in gray correspond to the typical TEP components, curve shadings are \pm 1 SEM. Black line below the traces indicates the period of the statistically significant difference. The vertical dashed line indicates the time of the TMS pulse (For interpretation of the references to colour in this figure legend, the reader is referred to the Web version of this article.)
Table 2

Results from the statistically significant clusters, cluster based t-tests, comparing POST vs. BASEINE with TEP amplitudes and spectral power of induced oscillations in the different frequency bands as dependent measures.

\begin{tabular}{|c|c|c|c|c|}
\hline Conditions & Cluster Latency & Degrees of Freedom & $\mathrm{t}$-value & $\mathrm{p}$-value \\
\hline \multicolumn{5}{|c|}{ TEP: $t$-test POST vs, BASELINE } \\
\hline$\# 1$ & $90-200 \mathrm{~ms}$ & 21 & -3.05 & 0.006 \\
\hline \multirow{2}{*}{\multicolumn{5}{|c|}{ Induced Oscillations: $t$-test POST vs. BASELINE }} \\
\hline & & & & \\
\hline Theta & $70-320 \mathrm{~ms}$ & 21 & -3.91 & $<0.001$ \\
\hline Gamma & $50-80 \mathrm{~m} \mathrm{~s}$ & 21 & -3.52 & 0.002 \\
\hline \multicolumn{5}{|l|}{ \#3 } \\
\hline Theta & $80-350 \mathrm{~ms}$ & 21 & -2.21 & 0.038 \\
\hline Gamma & $50-110 \mathrm{~ms}$ & 21 & -2.71 & 0.013 \\
\hline \multicolumn{5}{|l|}{$\# 4$} \\
\hline Theta & $130-280 \mathrm{~m} \mathrm{~s}$ & 21 & -3.13 & 0.005 \\
\hline Gamma & $50-120 \mathrm{~ms}$ & 21 & -3.35 & 0.003 \\
\hline
\end{tabular}

Table 3

Results from the cluster based one-way ANOVAs comparing the 5 tDCS condition Dependent variables were the BASELINE measurements and the Post-Baseline differences $(\Delta)$ of the TEP amplitudes and spectral power of induced oscillations in the different frequency bands.

\begin{tabular}{|c|c|c|c|c|}
\hline & Cluster Latency & Degrees of Freedom & F-value & p-value \\
\hline \multicolumn{5}{|c|}{ BASELINE TEP } \\
\hline & $120-190 \mathrm{~m} \mathrm{~s}$ & 4,84 & 0.831 & 0.509 \\
\hline \multicolumn{5}{|c|}{ BASELINE Induced Oscillations } \\
\hline Theta & -no clusters found- & & & \\
\hline Alpha & $270-320 \mathrm{~ms}$ & 4,84 & 0.804 & 0.526 \\
\hline Beta & $200-240 \mathrm{~ms}$ & 4,84 & 0.759 & 0.548 \\
\hline Gamma & -no clusters found- & & & \\
\hline \multicolumn{5}{|l|}{$\triangle$ TEP } \\
\hline & $90-200 \mathrm{~m} \mathrm{~s}$ & 4,84 & 1.98 & 0.104 \\
\hline \multicolumn{5}{|c|}{$\Delta$ Induced Oscillations } \\
\hline Theta & $90-310 \mathrm{~m} \mathrm{~s}$ & 4,84 & 0.86 & 0.417 \\
\hline Alpha & $80-120 \mathrm{~m} \mathrm{~s}$ & 4,84 & 0.99 & 0.413 \\
\hline Beta & $90-170 \mathrm{~m} \mathrm{~s}$ & 4,84 & 0.79 & 0.532 \\
\hline Gamma & $30-120 \mathrm{~m} \mathrm{~s}$ & 4,84 & 1.09 & 0.382 \\
\hline
\end{tabular}

\subsection{TMS-induced oscillations}

No significant difference of BASELINE measurements between tDCS conditions was found in any frequency band. Pairwise cluster based analysis revealed that three tDCS interventions resulted in changes of TMS-induced oscillations. TMS-induced spectral power was reduced in the theta and gamma bands after interventions \#1, \#3 and \#4 (Fig. 5 and Table 2). However, there was no significant difference comparing the changes POST minus BASELINE $(\Delta \mathrm{In}-$ duced Oscillations) across conditions, in any of the frequency bands (Table 3). Descriptive statistics and effect sizes of the POST vs. BASELINE data within tDCS conditions, and $\triangle$ Induced Oscillations of all tDCS conditions in comparison to sham are shown in the Supplementary Material (Tables S5 and S6).

\section{Discussion}

The objective of this study was to investigate changes in cortica responses after interventional stimulation of the DLPFC using tDCS with different stimulation parameters, as typically applied in clinical trials targeting the prefrontal cortex. We hypothesized that tDCS would lead to changes in cortical activity and responsivity measured by rs-EEG and TMS-EEG, with effects depending on polarity, current strength and electrode placement (cephalic vs. extracephalic return electrode)

Firstly, our results failed to show any change in rs-EEG spectral power in any analyzed frequency band, regardless of the tDCS

Please cite this article in press as: Gordon PC, et al., Modulation of cortical responses by transcranial direct current stimulation of dorsolateral prefrontal cortex: A resting-state EEG and TMS-EEG study, Brain Stimulation (2018), https://doi.org/10.1016/j.brs.2018.06.004 
\#1 1.5mA, anode left-DLPFC,
cathode right-DLPFC
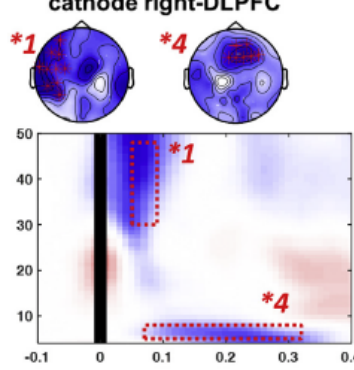

\#2 $1.5 \mathrm{~mA}$ cathode left-DLPFC,

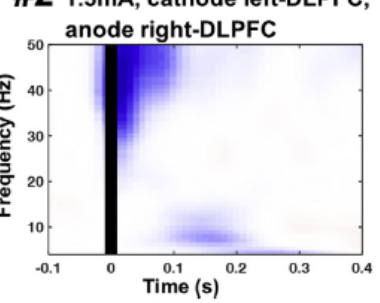

\#3 $0.5 \mathrm{~mA}$, anode left-DLPFC cathode right-DLPFC
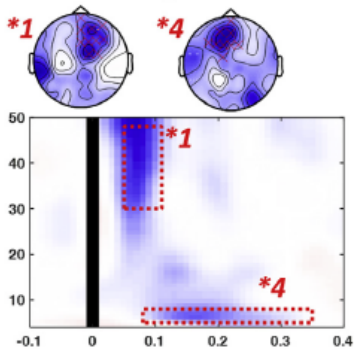

\#5 sham
\#4 1.5mA, anode left-DLPFC, cathode deltoid $\mathrm{m}$.

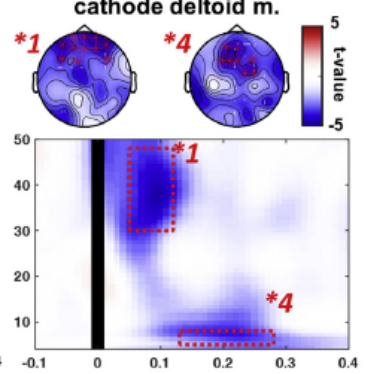

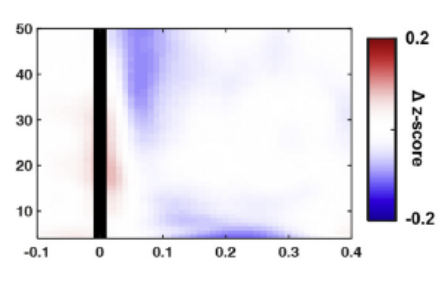

Fig. 5. Topoplots show the distribution of the t-values of the cluster statistics comparing the power of the induced oscillations between POST and BASEUNE. tDCS interventions \#1, \#3 and \#4 resulted in statistically significant clusters (highlighted in red ${ }^{\mathrm{x}}$ for $\mathrm{p}<0.05$ and ${ }^{*}$ for $\mathrm{p}<0.01$ ). The time-frequency plots below show the difference between POST and BASELINE ( $\triangle$-score), averaged across subjects and channels. Time and frequency where statistically significant clusters were found are highlighted by red dotted rectangles. (For interpretation of the references to colour in this figure legend, the reader is referred to the Web version of this article)

parameters used. Indeed, a meta-analysis of EEG spectral power changes after tDCS over prefrontal cortex revealed little consistency across findings, also providing insufficient evidence in support of any impact of tDCS on rs-EEG using the current parameters [23].

The TEP analyses suggested a significant decrease in amplitude following $1.5 \mathrm{~mA}$ anodal left-DLPFC tDCS, corresponding to reduction of the N120 amplitude over parietal cortex. Specifically, the difference was found in a broad latency range from 90 to $200 \mathrm{~m}$ s that extended beyond the typical duration of the N120. A decrease in TEP amplitude in this latency range has been associated to paired-pulse TMS induced states of cortical inhibition in the DLPFC [50], whereas the opposite effect was found during states of cortical facilitation [51]. Studies targeting the motor cortex also demonstrated a decrease in late TEP amplitudes in the latency range of $90-150 \mathrm{~ms}$ under the effect of GABA agonists [52,53]. These reports suggest that our findings are compatible with anoda tDCS promoting cortical inhibition in posterior cortical regions. Nevertheless, our study did not show any significant change in TEP amplitudes when comparing the POST vs. BASELINE TEP amplitude differences across conditions, including sham, which is in accord to previous nil results obtained from tDCS to the DLPFC using the bipolar montage [29].

Analysis of the TMS-induced oscillatory responses suggested a significant reduction in the power of induced oscillations in the prefrontal cortex. Specifically, interventions that had the anode placed over the left DLPFC led to a significant reduction of the power of induced theta and gamma oscillations. A similar result has also been reported by Hill et al. [29], showing mostly reduction in post-stimulus gamma oscillation power in response to tDCS intervention. Statistical testing comparing the change in cortical responses (BASELINE vs. POST) between all the conditions did not reveal any statistical significant difference, suggesting that the change in cortical responsivity after the tDCS interventions, including sham, was comparable across conditions. This is also in line with results from Hill et al. (2017), as significant changes in comparison to sham were observed following HD-tDCS, but not when using the bipolar montage.

Different results from standard bipolar and HD-tDCS might be explained by different current densities. Higher current density was associated with increased cortical modulation and, consequently, is recommended to be considered an independent stimulation parameter [54]. Accordingly, Hill et al. [29] proposed that the standard bipolar montage provided low current density $\left(1.0 \mathrm{~mA}\right.$, with current density of $\left.0.08 \mathrm{~mA} / \mathrm{cm}^{2}\right)$, which was not capable of yielding long-range cortical modulation observed in TEP analysis. Although the montage we used in our study was designed to emulate bipolar stimulation with similar current intensities $(0.5 \mathrm{~mA}$ and $1.5 \mathrm{~mA})$, the yielded current densities (respectively $0.16 \mathrm{~mA} / \mathrm{cm}^{2}$ and $0.47 \mathrm{~mA} / \mathrm{cm}^{2}$ ) were much higher than those produced by standard bipolar montages (respectively $0.02 \mathrm{~mA} / \mathrm{cm}^{2}$ and $0.06 \mathrm{~mA} / \mathrm{cm}^{2}$, for a $5 \times 5 \mathrm{~cm}$ set of electrodes). Still, despite the increased current density in our study, we did not find any differences in the effects following any of the active stimulation conditions and sham. A further difference is related to cortical electric field vectors, with bipolar montages mostly generating radial fields, as current runs from one hemisphere to the other, leading to polarization along the somatodendritic axes of radially oriented neurons, and HD-tDCS montages mostly generating tangential fields, leading to polarization of neuronal components parallel to the cortical surface, resulting in different profiles of modulation 
that might add to the different results [55,56]. Furthermore, electric field distributions over the cortex in both standard bipolar and in this study's montage are diffusely spread in frontal cortex, while it has been demonstrated that HD-tDCS montages are capable of more focal stimulation with a significantly higher electric potentia in the targeted region [55]. Higher electric potential applied to the DLPFC, the target of both tDCS and the probing TMS pulses for evoked responses, might have been responsible for a significant change in TMS-EEG cortical responses observed following HD-tDCS [29], whereas the lower electric field potential provided by bipolar and present montages might not be sufficient to promote such changes.

Negative results obtained in our study when comparing the effects across conditions are contrary to our previous expectations, given reports on TMS-evoked EEG responses following moto cortex-tDCS showing cortical facilitation following anodal stimulation, and cortical inhibition following cathodal stimulation [25] Higher current densities in both 0.5 and $1.5 \mathrm{~mA}$ anodal conditions might explain the trend towards higher facilitation, as predicted from motor cortex studies. However, the comparison with sham suggested no different neuromodulatory effects from tDCS what soever. Likewise, cathodal stimulation should have resulted in either inhibition, as in most tDCS-motor cortex studies [12,13,25] or facilitation, as seen in reports applying cathodal stimulation with higher current intensities [17]. Nonetheless, it is possible that neuromodulation from tDCS over the prefrontal cortex has different effects than over the motor cortex, when the same parameters are applied. Also, there is a lack of a linear relation between current intensities and physiological effects on the motor cortex, which adds a limitation to a direct comparison with the effects on prefrontal cortex [17,57]. A previous report also showed different neuromodulation profiles in the prefrontal cortex, compared to motor cortex, following repetitive TMS [58], suggesting differential effects to a given set of stimulation parameters in different cortical targets.

The present study has limitations that should be addressed in further experiments. As previously mentioned, most of the clinical evidence for tDCS application is derived from studies using bipolar montages. This imposes a challenge for concomitant EEC measurement of the effects of stimulation on the targeted area, as the placement of a large tDCS electrode on the scalp precludes EEG data collection from that specific target. The use of the present montage, with small interleaved tDCS electrodes, is a solution to overcome this limitation. However, despite havin similar current intensities and yielding a comparable electric field model, differences in the resulting current densities in the brain might have led to different effects at the cortical level compared to those with standard electrode montages. Thus, it is not possible to directly translate the results obtained in this study to those of conventional bipolar tDCS. Also, the effects on cortical modulation were investigated immediately after a single tDCS session, whereas therapeutic effects of tDCS of the DLPFC are typically obtained only after a series of repeated sessions. Measuring modulation of TMS-EEG cortical responses after a proper tDCS trial would be a meaningful way of determining the impact of the treatment on cortical activity, as well as pinpointing the actual markers related to clinical improvement, which might be of great importance to further develop effective non-invasive brain stimulation treatment for neuropsychiatric conditions $[59,60]$. Finally, the exploratory nature of the present study might have increased the likelihood for negative findings, give the multiple variables and conditions included. Future studies targeting specific tDCS protocols with higher likelihood of inducing cortical change might be able to detect specific changes in cortical excitability measurements.

\section{Conclusions}

Our results provide exploratory evidence for changes in cortical activity and reactivity following single tDCS sessions targeting the left DLPFC, using tDCS parameters commonly applied in clinical trials. Conditions that involved higher current intensities $(1.5 \mathrm{~mA})$ and anodal tDCS of the left DLPFC showed significant changes in cortical reactivity in the POST vs. BASELINE comparison, but these changes were no longer significant if comparing them with those in the other tDCS conditions including sham. Also, significant changes in cortical activity or reactivity might be observed following a series of tDCS sessions targeting the prefrontal cortex, in parallel to cognitive and/or clinical outcomes, and might provide further information on the effects of tDCS of the prefrontal cortex, but this will need to be tested in future studies.

\section{Funding}

P.C.G. and C.Z. are supported through a German Federal Ministry for Economic Affairs and Energy (03EFJBW169) of EXIST Transfer of Research Grant. C.Z. acknowledges support from the Clinician Scientist Program at the Faculty of Medicine at the gs2:University of Tübingen. This study was further supported by DFG grant ZI 542/71 (to U.Z.). This research was also supported with funding from the Federal Ministry of Education and Research of Germany through the MOTOR-BIC project (BMBF, grant 13GW0053A).

\section{Conflicts of interest}

A.R.B. is recipient of a CAPES/Alexander von Humboldt fellowship award for experienced researchers and a consultant for the Neurocare group GmbH (Munich, Germany). The remaining authors declare that the research was conducted in the absence of any competing financial interest.

\section{Appendix A. Supplementary data}

Supplementary data related to this article can be found at https://doi.org/10.1016/j.brs.2018.06.004.

\section{References}

[1] Philip NS, Nelson BG, Frohlich F, Lim KO, Widge AS, Carpenter LL. Low-intensity transcranial current stimulation in psychiatry, Am J Psychiat 2017:174(7):628-39.

[2] Bikson M, Grossman P, Thomas C, Zannou AL, Jiang J. Adnan T, et al, Safety of transcranial direct current stimulation: evidence based update 2016. Brain Stimul 2016:9(5):641-61.

[3] Antal A, Alekseichuk I, Bikson M, Brockmoller J, Brunoni AR, Chen R, et al. Low intensity transcranial electric stimulation: safety, ethical legal regulatory and application guidelines. Clin Neurophysiol 2017:128(9):1774-809.

[4] Brunoni AR, Moffa AH, Sampaio-Junior B, Borrione L, Moreno ML Fernandes RA, et al. Trial of electrical direct-current therapy versus escitalopram for depression. N Engl J Med 2017:376(26):2523-33,

[5] Lefaucheur JP, Antal A, Ayache SS, Benninger DH, Brunelin J, Cogiamanian F, et al. Evidence-based guidelines on the therapeutic use of transcranial direct current stimulation (tDCS). Clin Neurophysiol 2017;128(1):56-92.

[6] Brunoni AR, Vanderhasselt MA. Working memory improvement with noninvasive brain stimulation of the dorsolateral prefrontal cortex: a systematic review and meta-analysis, Brain Cognit 2014;86:1-9.

77] Dedoncker J, Brunoni AR, Baeken C, Vanderhasselt MA. A systematic review and meta-analysis of the effects of transcranial direct current stimulation (tDCS) over the dorsolateral prefrontal cortex in heal thy and neuropsychiatric samples: influence of stimulation parameters. Brain Stimul 2016;9(4):

[8] Huang YZ, Lu MK, Antal A, Classen J, Nitsche M, Ziemann U, et al, Plasticity induced by non-invasive transcranial brain stimulation: a position paper. Clin Neurophysiol 2017;128(11):2318-29.

[9] Woods A], Antal A, Bi kson M, Boggio PS, Brunoni AR, Celnik P, et al. A technical guide to tDCS, and related non-invasive brain stimulation tools. Cin Neurophysiol 2016;127(2):1031-48; 


\section{ARTICLE IN PRESS}

[10] Purpura DP, McMurtry JG. Intracellular activities and evoked potential changes during polarization of motor cortex.J Neurophysiol 1965:28:166-85. uniform extracellular DC electric fields on excitability in rat hippocampal slices in vitro. J Physiol 2004;557(Pt 1):175-90.

[12] Nitsche MA, Paulus W. Excitability changes induced in the human motor cortex by weak transcranial direct current stimulation. J Physiol 2000:527( Pt cortex by
3):633-9.

[13] Nitsche MA, Seeber A, Frommann K, Klein $\propto$ C, Rochford C, Nitsche MS, et al, Modulating parameters of excitability during and after transcranial direct current stimulation of the human motor cortex. J Physiol 2005:568(Pt 1): 291-303.

[14] Nitsche MA, Nitsche MS, Klein CC, Tergau F, Rothwell JC, Paulus W. Level of action of cathodal DC polarisation induced inhibition of the human motor cortex. Clin Neurophysiol 2003;114(4):600-4.

[15] Nitsche MA, Cohen LG, Wassermann EM, Priori A, Lang N, Antal A, et al. Transcranial direct current stimulation: state of the art 2008. Brain Stimul 2008;1(3):206-23.

[16] Monte-Silva K, Kuo MF, Hessenthaler S, Fresnoza S, Liebetanz D, Paulus W, et al. Induction of late LTP-like plasticity in the human motor cortex by

[17] Batsikadze G, Moliadze V, Paulus W, Kuo MF, Nitsche MA, Partially non-linear stimulation intensity-dependent effects of direct current stimulation on mostimulation intensity-dependent effects of direct current stimulation
tor cortex excitability in humans. J Physiol 2013;591(7):1987-2000. [18] Antal A, Terney D, Poreisz C, Paulus W. Towards unravelling task-related Eur J Neurosci 2007;26(9):2687-91.

[19] Miller J, Berger B, Sauseng P. Anodal transcranial direct current stimulation (tDCS) increases frontal-midline theta activity in the human EEG: a preliminary investigation of non-invasive stimulation. Neurosci Lett 2015;58: $114-9$.

[20] Accornero N, Capozza M, Pieroni L, Pro S, Davi L, Mecarelli O. EEG mean frequency changes in healthy subjects during prefrontal transcranial direct current stimulation. J Neurophysiol 2014;112(6):1367-75.

[21] Maeoka H, Matsuo A, Hiyamizu M, Morioka S, Ando H. Influence of transcranial direct current stimulation of the dorsolateral prefrontal cortex on pain related emotions: a study using electroencephalographic power spectrum analysis. Neurosci Lett 2012;512(1):12-6.

[22] Boonstra TW, Nikolin S, Meisener AC, Martin DM, Loo CK. Change in mean frequency of resting-state electroencephalography after transcranial direct

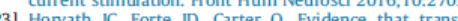

that transcranial direct current stimulation (tDCS) generates little-to-no reliable neurophysiologic effect beyond MEP amplitude modulation in heal thy

4) review. Neuropsychologia 2015;66:213-36.

ilmoniemi RJ, Virtanen J, Ruohonen J, Karhu J, Aronen HJ, Naatanen R, et al. cournectivity. Neuroreport 1997;8(16):3537-40.

[25] Pellicciari MC, Brignani D, Miniussi C. Excitability modulation of the motor system induced by transcranial direct current stimulation: a multimodal approach, Neuroimage 2013;83:569-80.

[26] Hill AT, Rogasch NC, Fitzgerald PB, Hoy KE. TMS-EEG: a window into the neurophysiological effects of transcranial electrical stimulation in non-motor brain regions. Neurosci Biobehav Rev 2016;64:175-84,

[27] Rogasch NC, Fitzgerald PB. Assessing cortical network properties using TMSEEG. Hum Brain Mapp 2013;34(7):1652-69,

[28] Romero Lauro Ly, Rosanova M, Mattavelli G, Convento S, Pisoni A, Opitz A, et al. TDCS increases cortical excitability: direct evidence from TMS-EEG. Cortex 2014;58:99-11.

[29] Hill AT, Rogasch NC, Fitzgerald PB, Hoy KE. Effects of prefrontal bipolar and high-definition transcranial direct current stimulation on cortical reactivity

[30] Oldfield RC. The assessment and analysis of handedness: the Edinburgh in ventory, Neuropsychologia 1971:9(1):97-113.

[31] Gandiga PC, Hummel FC, Cohen LG. Transcranial DC stimulation (tDCS): a tool for double-blind sham-controlled clinical studies in brain stimulation. Clin Neurophysiol 2006;117(4):845-50.

[32] Brunoni AR, Moffa AH, Fregni F, Palm U, Padberg F, Blumberger DM, et al, Transcranial direct current stimulation for acute major depressive episodes: meta-analysis of individual patient data. Br J Psychiatry 2016;208(6):522-31. neuronavigated methods to target the left dorsolateral pre-frontal cortex with symmetnic bicephalic transcranial direct current stimulation (tDCS). Brain Stimul 2015;8(3):590-602.

34] Rusjan PM, Barr MS, Farzan F, Arenovich T, Maller Il, Fitzgerald PB, et al. Optimal transcranial magnetic stimulation coil placement for targeting the dorsolateral prefrontal cortex using novel magnetic resonance image-guided neuronavigation. Hum Brain Mapp 2010;31(11):1643-52 [35] Roy A, Baxter B, He B. High-definition transcranial direct current stimulation synchronization: a simultaneous tDCS-EEG study. IEEE Trans Biomed Eng

2014;61(7): 1967-78.
[36] Sood M, Besson P, Muthalib M, Jindal U, Perrey S, Dutta A, et al. NIRS-EEG joint imaging during transcranial direct current stimulation: online parameter

[37] Windhoff M, Opitz A, Thielscher A. Electric field calculations in brain stimulation based on finite elements; an optimized processing pipeline for the the Hum Brain Mapp 2013;34(4):923-35,

[38] Groppa S, Oliviero A, Eisen A, Quartarone A, Cohen LG, Mall V, et al. A practical guide to diagnostic transcranial magnetic stimulation: report of an IFCN committee, Cin Neurophysiol 2012;123(5):858-82.

[39] Oostenveld R, Fries P, Maris E, Schoffelen JM. FieldTrip: open source software for advanced analysis of MEG, EEG, and invasive electrophysiological data. Comput Intell Neurosci 2011;2011. 156869.

[40] Hyvarinen A. Fast and robust fixed-point algorithms for independent component analysis. IEEE Trans Neural Network 1999;10(3):626-34.

[41] Perrin F, Pernier J, Bertrand O, Echallier JF. Spherical splines for scalp potential and current density mapping. Electroencephalogr Clin Neurophysiol

1989;72(2):184-7.
[42] Rogasch NC, Thomson RH, Farzan F, Fitzgibbon BM, Bailey NW, HernandezPavon JC, et al. Removing artefacts from TMS-EEG recordings using independent component analysis: importance for assessing prefing

[43] Rogasch NC, Sul livan C, Thomson RH, Rose NS, Bailey NW, Fitzgerald PB, et al. Analysing concurrent transcranial magnetic stimulation and electroencephalographic data: a review and introduction to the open-source TESA software. Neuroimage 2017:147:934-51

[44] Tallon-Baudry C, Bertrand O. Oscillatory gamma activity in humans and its role in object representation. Trends Cognit Sci 1999;3(4):151-62

[45] Pellicciari MC Veniero D, Miniussi C. Characterizing the cortical oscillatory response to TMS pulse. Front Cell Neurosci 2017;11:38.

[46] Premoli I, Bergmann TO, Fecchio M, Rosanova M, Biondi A, Belardinelli P, et al, The impact of GABAergic drugs on TMS-induced brain oscillations in human motor cortex. Neuroimage 2017;163:1-12

[47] Grandchamp R, Delome A. Single-trial nomalization for event-related spectral decomposition reduces sensitivity to noisy trials. Front Psychol 2011;2:236

[48] Maris E, Oostenveld R. Nonparametric statistical testing of EEG- and MEGdata. J Neurosci Meth 2007;164(1):177-90.

[49] Bai S, Dokos S, Ho KA, Loo C A computational modelling study of transcranial used in depression. Neuroimage

50] Daskalakis ZJ, Farzan F, Barr MS, Maller ע, Chen R, Fitzgerald PB. Long-interval cortical inhibition from the dorsolateral prefrontal cortex: a TMS-EEG study.

[51] Cash RF, Noda Y, Zomorrodi R, Radhu N, Farzan F, Rajii TK, et al. Characterization of glutamatergic and GABAA-mediated neurotransmission in motor and dorsolateral prefrontal cortex using

[52] Premoli I, Gastellanos N, Rivolta D, Belardinelli P, Bajo R, Zipser C, et al. TMSEEG signatures of GABAergic neurotransmission in the human cortex. J Neurosci 2014;34(16):5603-12,

[53] Premoli I, Rivolta D, Espenhahn S, Castellanos N, Belardinelli P, Ziemann U, et al. Characterization of GABAB-receptor mediated neurotransmission in the human cortex by paired-pulse TMS-EE,. Neuroimage 2014,103:152-62.

[54] Nitsche MA, Doemkes S, Karakose T, Antal A, Liebetanz D, Lang N, et al. Shaping the effects of transcranial direct current stimulation of the human

55] Kuo HI, Bikson M, Datta A, Minhas P, Paulus W, Kuo MF, et al. Comparing cortical plasticity induced by conventional and high-definition $4 \times 1$ ring

of al allar effects of acute direct current stimulation: som

7] Jamil A, Batsikadze G, Kuo HI, Labruna L, Hasan A, Paul us W, et al. Systematic induced by transcranial direct current stimulation. J Physiol 2017:595(4): 1273-88.

[58] Chung SW, Lewis BP, Rogasch NC, Saeki T, Thomson RH, Hoy KE, et al. Demonstration of short-term plasticity in the dorsolateral prefrontal cortex
with theta burst stimulation: a TMS-EEG study. Cin Neurophysiol 2017;128(7):1117-26.

[59] Chung T, Tittgemeyer M, Feldstein Ewing SW. Introduction to the Special Issue: using neuroimaging to probe mechanisms of behavior change. Neuroimage 2017:151:1-3.

[60] Loo CK, Husain MM, McDonald WM, Aaronson S, O ReardonJP, Alonzo A, et al. International randomized-controlled trial of transcranial Direct Current
Stimulation in depression. Brain Stimul 2018;11(1):125-33. 